Supporting Information for

\title{
Mechanistic Study of Reduction and Initiation over Vanadium-oxide Polyethylene Catalyst
}

Kaimin Liu, ${ }^{a}$ Zhen Liu, ${ }^{* a}$ Ruihua Cheng, ${ }^{a}$ Xuelian He ${ }^{a}$ and Boping Liu ${ }^{* b}$

${ }^{a}$ School of Chemical Engineering, East China University of Science and Technology, Shanghai 200237,

China

${ }^{b}$ College of Materials and Energy, South China Agricultural University, Guangzhou 510642, China

${ }^{*}$ Corresponding author:

Zhen Liu, Email: liuzhen@ecust.edu.cn

Boping Liu, Email: boping@scau.edu.cn 


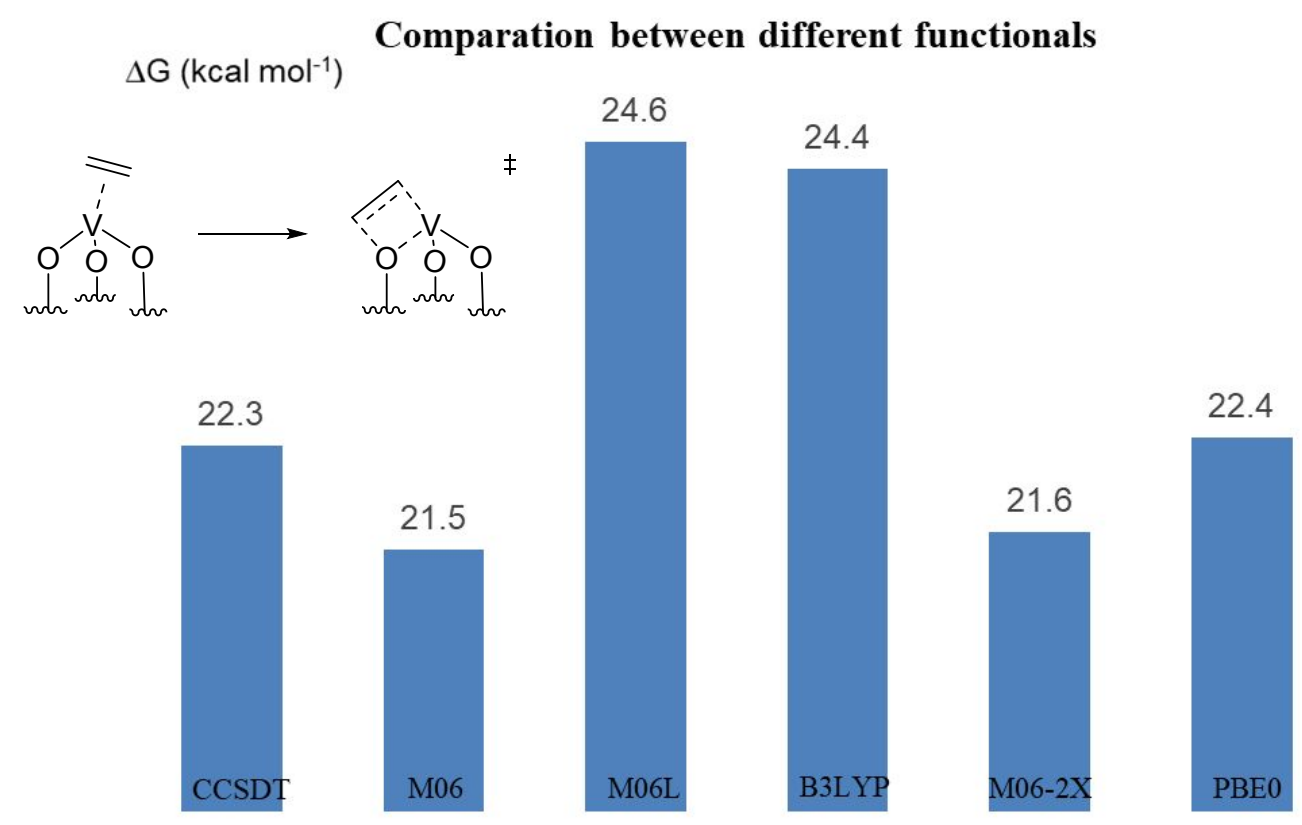

Figure S1. Activation energy barriers $\left(\Delta \mathrm{G}^{\dagger}, \mathrm{kcal} / \mathrm{mol}\right)$ for transition states associated with ethylene insertion. The Gibbs free energy with $\operatorname{CCSD}(\mathrm{T})$ is given by $\Delta \mathrm{G}=\Delta \mathrm{E}_{\mathrm{SCF}-\mathrm{CCSD}(\mathrm{T})}+\Delta \mathrm{G}_{\text {corr }}+$ $\Delta \mathrm{G}_{\text {solv }}$ and the Gibbs free energy with other functionals is given by $\Delta \mathrm{G}=\Delta \mathrm{E}_{\mathrm{SCF}-\text { solv }}+\Delta \mathrm{G}_{\text {corr }}$. 


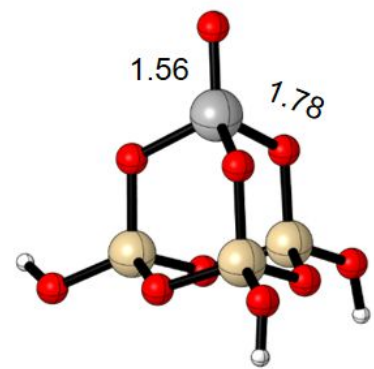

${ }^{1}$ Model A

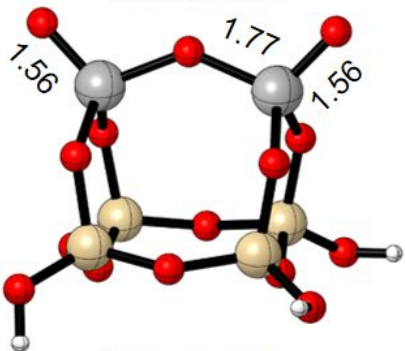

${ }^{1}$ Model B

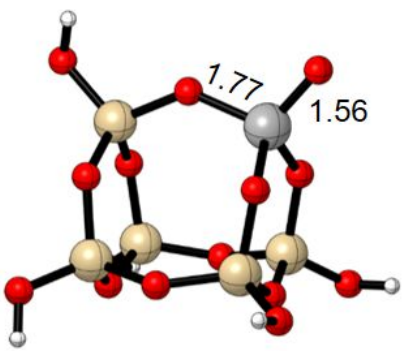

${ }^{1}$ Model C

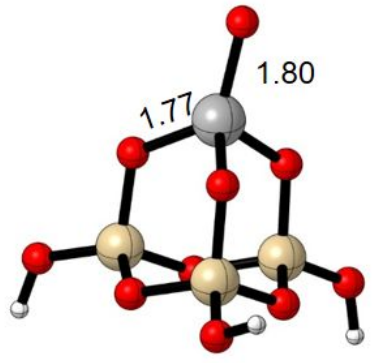

${ }^{3}$ Model A

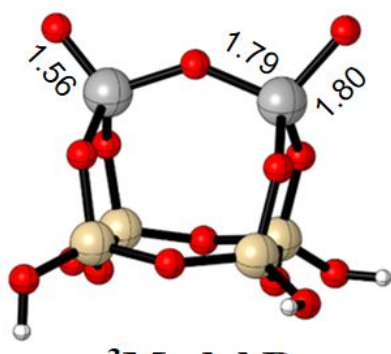

${ }^{3}$ Model B

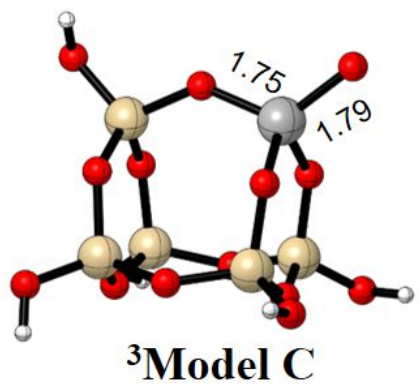

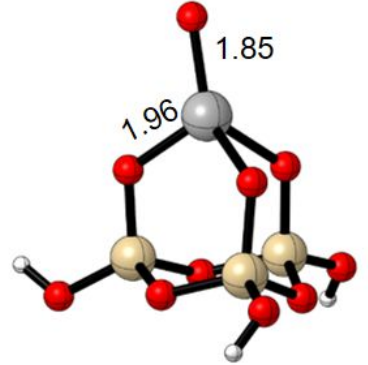

${ }^{5}$ Model A
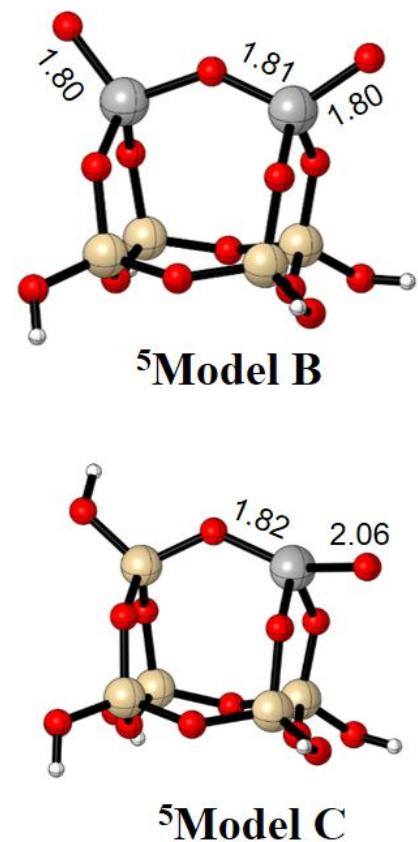

Figure S2. Geometric features of the optimized model catalysts A, B and C with different spin multiplicity. The bond lengths are in $\AA$ (red atoms represent oxygen atoms, grey atoms represent vanadium atoms, pale yellow atoms represent silicon atom) 
Table S1. NBO charges on vanadium center and energy of different models with different spin multiplicity

\begin{tabular}{ccccccc}
\hline \multirow{2}{*}{ Natural charge } & Singlet & $\begin{array}{c}\text { Energy (kcal } \\
\left.\mathrm{mol}^{-1}\right)\end{array}$ & Triplet & $\begin{array}{c}\text { Energy (kcal } \\
\left.\mathrm{mol}^{-1}\right)\end{array}$ & Quintet & $\begin{array}{c}\text { Energy (kcal } \\
\left.\mathrm{mol}^{-1}\right)\end{array}$ \\
\hline A & 0.92 & 0.0 & 1.09 & 60.8 & 1.21 & 121.5 \\
B & 0.94 & 0.0 & 1.09 & 59.0 & 1.11 & 117.9 \\
C & 0.95 & 0.0 & 1.10 & 59.4 & 1.22 & 115.6 \\
\hline
\end{tabular}



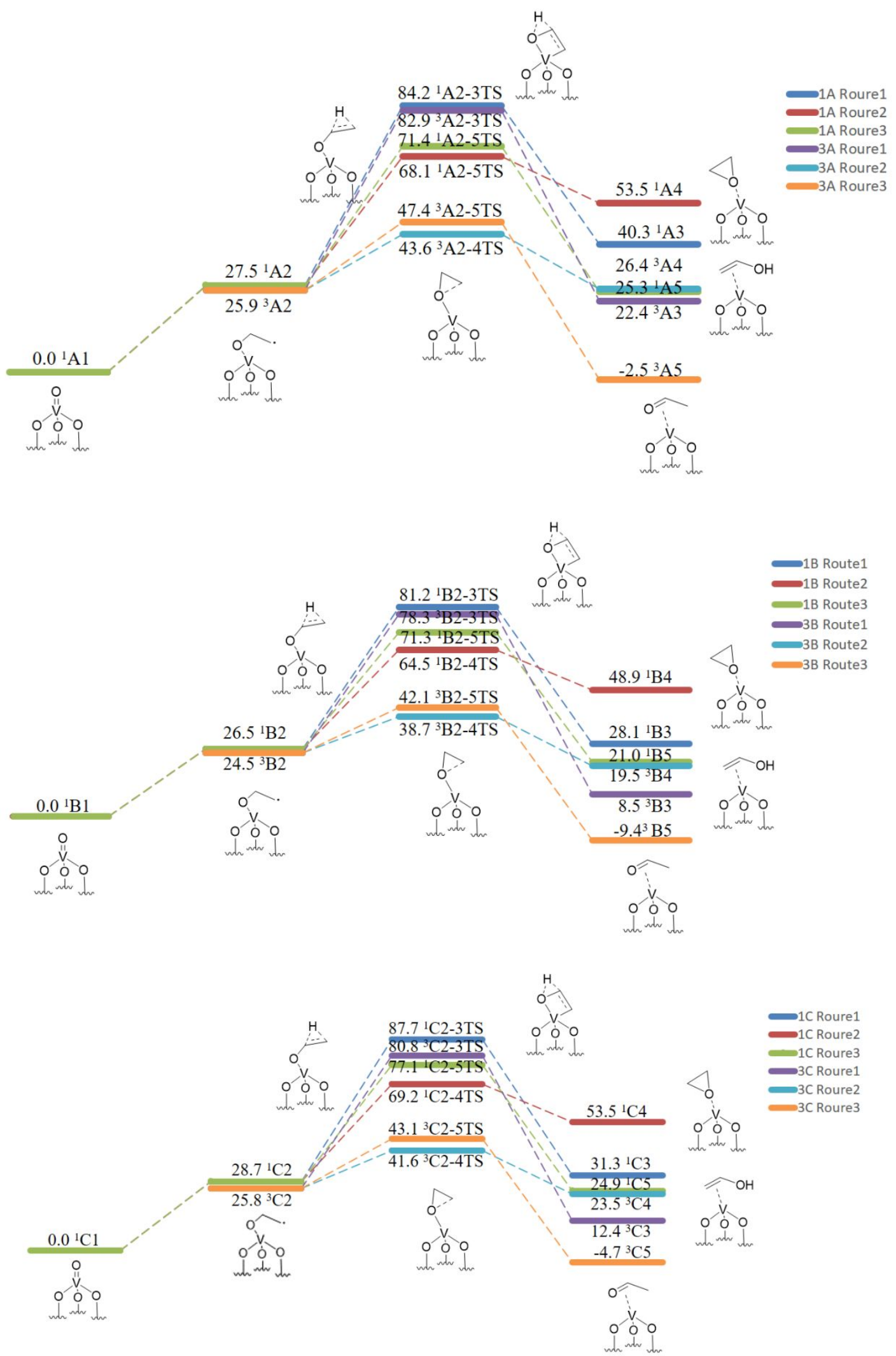

Figure S3. Relative Gibbs free energy profiles for $\left(\mathrm{kcal} \mathrm{mol}^{-1}\right)$ for reduction without co-catalyst over Model A, B and C. 


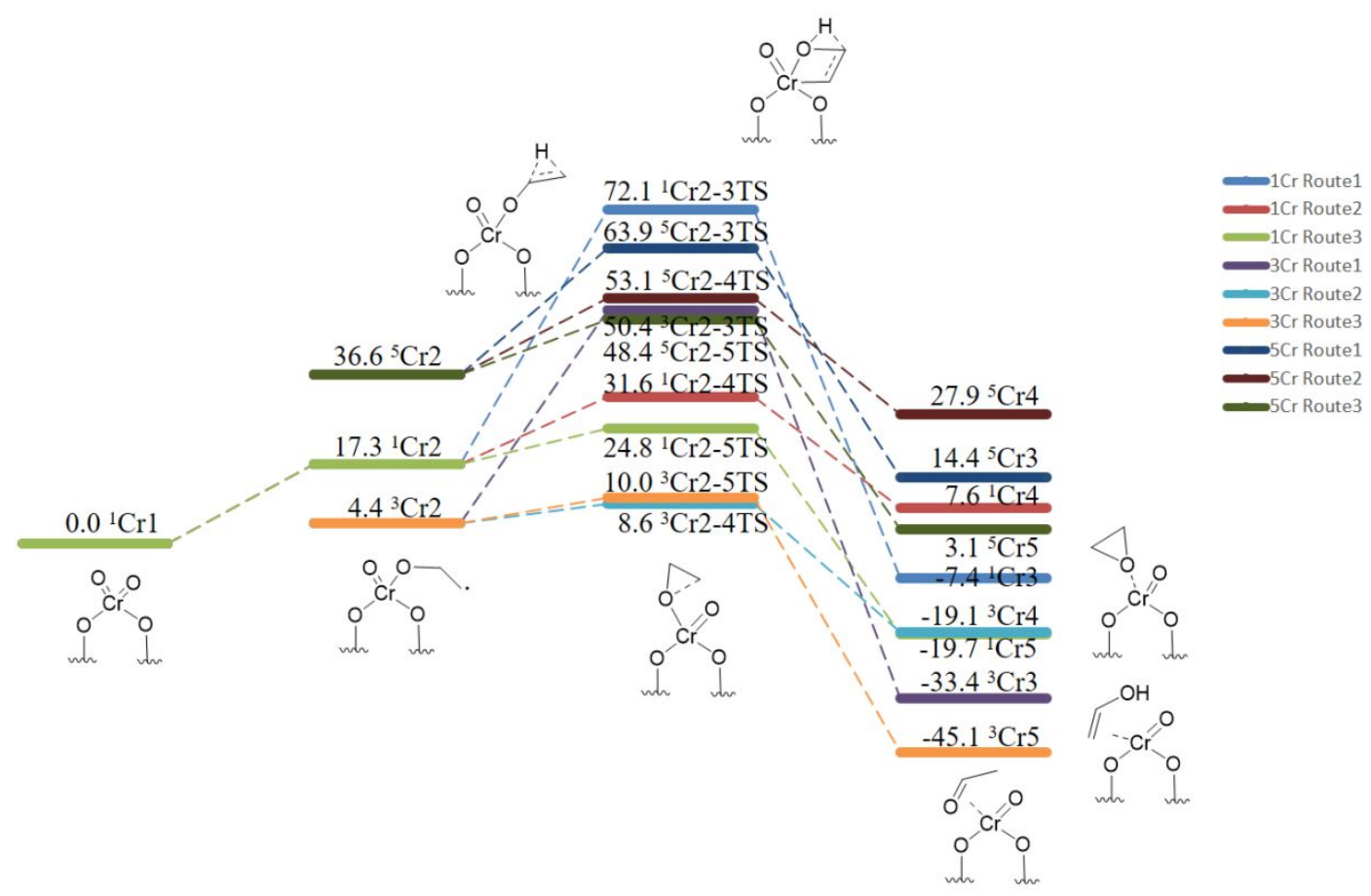

Figure S4. Relative Gibbs free energy profiles for $\left(\mathrm{kcal} \mathrm{mol}^{-1}\right)$ for reduction without co-catalyst over Cr(VI) Phillips catalyst. 
Table S2. Calculated reaction energies $\left(\mathrm{kcal} \mathrm{mol}^{-1}\right)$ for reduction when $\mathrm{AlEt}_{2} \mathrm{Cl}$ is taken as cocatalyst

\begin{tabular}{cccc}
\hline & 11 & ${ }^{3} 6(\mathrm{~b})$ & $37(\mathrm{~b})$ \\
\hline Model A & 0.0 & 4.0 & 38.0 \\
Model B & 0.0 & 3.0 & 33.1 \\
Model C & 0.0 & 6.8 & 35.8 \\
\hline
\end{tabular}

Table S3. Relative Gibbs free energies $\left(\mathrm{kcal} \mathrm{mol}^{-1}\right)$ of the different structures with different spin

\begin{tabular}{ccccc}
\hline & & Model A & Model B & Model C \\
\hline \multirow{3}{*}{ Structure 2 } & Singlet & 27.5 & 26.5 & 28.7 \\
& MECP & 37.0 & 33.0 & 37.3 \\
& Triplet & 25.9 & 24.5 & 25.8 \\
& Singlet & -0.9 & -1.1 & -0.6 \\
\multirow{3}{*}{ Structure 6 } & MECP & 7.9 & 6.7 & 10.4 \\
& Triplet & 1.5 & -0.9 & 2.8 \\
\hline
\end{tabular}




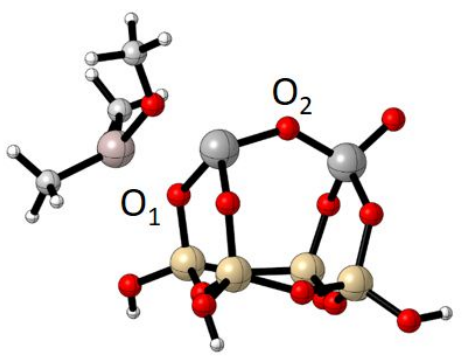

${ }^{3} \mathrm{~B} 7$

Free Energy: $-48.0 \mathrm{kcal} \mathrm{mol}^{-1}$

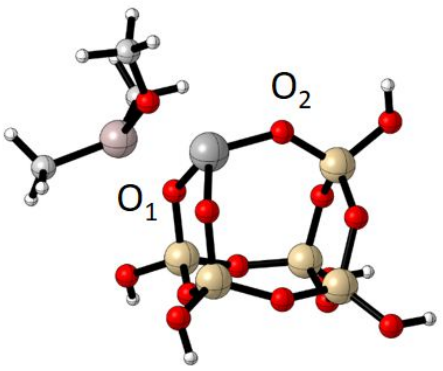

${ }^{3} \mathrm{C} 7$

Free Energy: $-44.7 \mathrm{kcal} \mathrm{mol}{ }^{-1}$

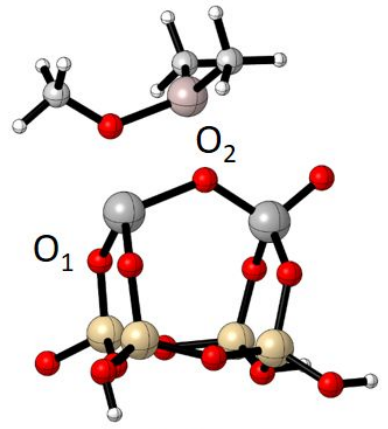

${ }^{3} \mathrm{~B} 7$ (II)

Free Energy: $-43.0 \mathrm{kcal} \mathrm{mol}^{-1}$

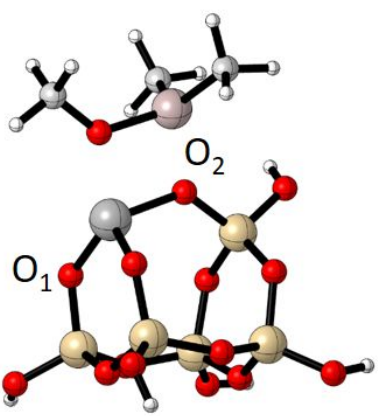

${ }^{3} \mathrm{C} 7$ (II)

Free Energy: $-41.3 \mathrm{kcal} \mathrm{mol}^{-1}$

Figure S5. Optimized structures of ${ }^{3} \mathrm{~B} 7$ and ${ }^{3} \mathrm{C} 7$ interacting with different oxygen atoms. 


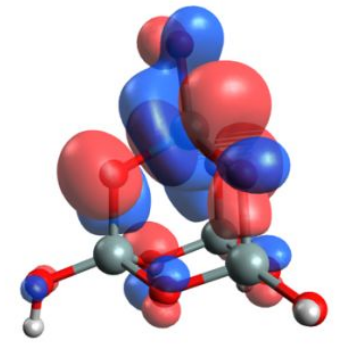

${ }^{3}$ Model A(LUMO)

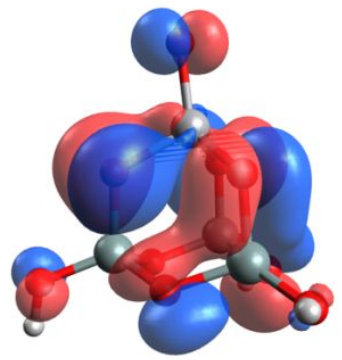

${ }^{3}$ Model A(HOMO)
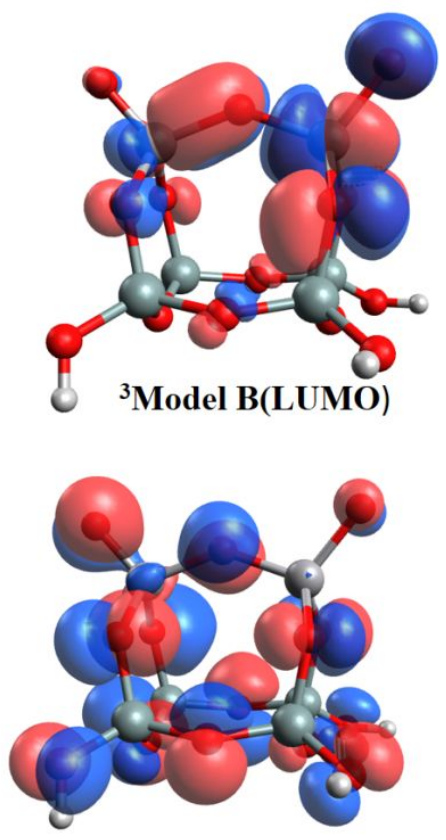

${ }^{3}$ Model B(HOMO)
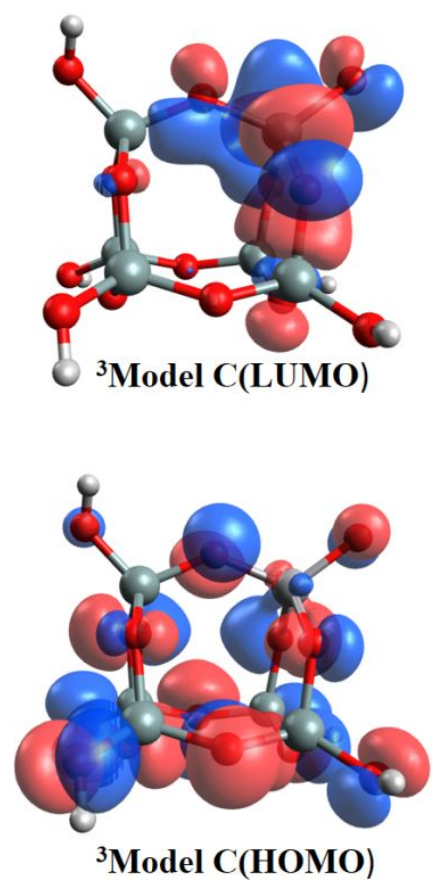

Figure S6. Frontier molecular orbitals of ${ }^{3} \operatorname{Model} X(X=A, B, C)$ : (top) LUMO; (bottom) HOMO 
List of Cartesian coordinates

$\begin{array}{lrrr}{ }^{1} \mathrm{~A} 1 & & & \\ \mathrm{~V} & -1.76474100 & 0.04324000 & -0.07748800 \\ 0 & -3.32138500 & 0.08565900 & -0.13999500 \\ 0 & -0.91669700 & 0.89365900 & -1.38753300 \\ 0 & -1.04024700 & -1.57869700 & -0.12806600 \\ 0 & -1.03876200 & 0.74639100 & 1.38514300 \\ \mathrm{Si} & 0.76312000 & 0.88340600 & -1.36138400 \\ \mathrm{Si} & 0.63522500 & -1.67658200 & -0.05669000 \\ \mathrm{Si} & 0.64544600 & 0.73996100 & 1.49763600 \\ 0 & 1.20331700 & 1.50199200 & 0.12761900 \\ 0 & 1.20310400 & -0.72834500 & -1.31051800 \\ 0 & 1.07732800 & -0.86558600 & 1.33641400 \\ 0 & 1.22495600 & 1.46402500 & 2.82993000 \\ \mathrm{H} & 0.77971100 & 1.30499500 & 3.67269700 \\ 0 & 1.35257400 & 1.70704200 & -2.62859400 \\ \mathrm{H} & 2.30211800 & 1.87988200 & -2.67351500 \\ 0 & 1.11060000 & -3.22710600 & -0.11333500 \\ \mathrm{H} & 2.05583300 & -3.42666000 & -0.09935000\end{array}$

\begin{tabular}{|c|c|c|c|}
\hline V & 1.43888900 & -0.10248500 & -0.00256000 \\
\hline 0 & 0.58749000 & - & -1 \\
\hline 0 & a. 58116590 & 152563390 & -0.00340800 \\
\hline 0 & 0.58861500 & -0.71909500 & 1.43146800 \\
\hline Si & -1.08789900 & -0.80118800 & -1.43728100 \\
\hline Si & -1.06524100 & 1.64954200 & 0.00019000 \\
\hline $\mathrm{Si}$ & -1.09373400 & -0.79927200 & 1.43449400 \\
\hline 0 & -1.54134000 & -1.52226400 & 0.00255900 \\
\hline 0 & -1.61261100 & 0.77651400 & -1.32518000 \\
\hline 0 & -1.60325400 & 0.78367300 & 1.33449300 \\
\hline 0 & -1.70125600 & -1.63142400 & 2.69409400 \\
\hline $\mathrm{H}$ & -1.31409800 & -1.47649500 & 3.56547900 \\
\hline 0 & -1.60651900 & -1.60094300 & -2.75502200 \\
\hline $\mathrm{H}$ & -2.52458400 & -1.90099900 & -2.77254000 \\
\hline 0 & -1.55797200 & 3.20250900 & -0.00132400 \\
\hline $\mathrm{H}$ & -2.50832000 & 3.37317400 & -0.02716000 \\
\hline 0 & 2.94506100 & -1.06347400 & 0.00743600 \\
\hline C & 3.98666600 & -0.08446700 & -0.01570700 \\
\hline $\mathrm{H}$ & 4.62961600 & -0.19941700 & 0.87473500 \\
\hline $\mathrm{H}$ & 4.60847300 & -0.22061300 & -0.91793400 \\
\hline C & 3.12481200 & 1.15098600 & -0.02146500 \\
\hline $\mathrm{H}$ & 3.07791300 & 1.75489700 & -0.93717100 \\
\hline
\end{tabular}


${ }^{1} \mathrm{~A} 2$-3TS

$\begin{array}{lrrr}\text { V } & -1.49716700 & 0.03498200 & -0.18827200 \\ \text { O } & -2.96540800 & -1.21401800 & -0.18472400 \\ \text { O } & -0.51130300 & -1.32905200 & -0.96196400 \\ \text { O } & -0.81938700 & 0.14762700 & 1.44405900 \\ \text { O } & -0.42219700 & 1.33658200 & -0.90008100 \\ \text { Si } & 1.11703900 & -1.44029100 & -0.73360100 \\ \text { Si } & 0.84849100 & 0.01702800 & 1.71161800 \\ \text { Si } & 1.22216600 & 1.37581000 & -0.75047200 \\ \text { O } & 1.83354700 & -0.05974800 & -1.34485100 \\ \text { O } & 1.35815000 & -1.35098700 & 0.93269800 \\ \text { O } & 1.50130100 & 1.32116000 & 0.90780700 \\ \text { O } & 1.87262000 & 2.69059400 & -1.46551100 \\ \text { H } & 2.81299800 & 2.64894600 & -1.68261100 \\ \text { O } & 1.81745000 & -2.75763200 & -1.39844500 \\ \text { H } & 1.32169900 & -3.58427800 & -1.33848900 \\ \text { O } & 1.22684000 & -0.05337700 & 3.29551700 \\ \text { H } & 1.07486300 & 0.73886000 & 3.82651400 \\ \text { C } & -3.51055200 & 0.13589700 & 0.35667200 \\ \text { H } & -3.56757600 & 0.20301800 & 1.44635700 \\ \text { H } & -4.12339100 & -0.85121400 & -0.12098200 \\ \text { C } & -3.18752000 & 1.14222800 & -0.62895700 \\ \text { H } & -3.53884700 & 1.00525500 & -1.65999800 \\ \text { H } & -3.11710900 & 2.18123200 & -0.28850400\end{array}$

$\begin{array}{lrrr}{ }^{1} \mathrm{~A} 2-4 \mathrm{TS} & & & \\ \mathrm{V} & 1.05117800 & 0.09305300 & -0.91290400 \\ 0 & 2.92142700 & -0.05022000 & -1.05483000 \\ 0 & 1.22476100 & -0.01030400 & 0.95011400 \\ 0 & 0.06657600 & -1.32551500 & -1.37930300 \\ 0 & 0.01592000 & 1.53570000 & -1.08305200 \\ \mathrm{Si} & -0.22176200 & -0.13159800 & 1.72952100 \\ \mathrm{Si} & -1.39145000 & -1.41459300 & -0.56694900 \\ \mathrm{Si} & -1.46504000 & 1.44527200 & -0.29103900 \\ 0 & -1.14456200 & 1.21448900 & 1.31958200 \\ 0 & -1.06262400 & -1.42984400 & 1.06811700 \\ 0 & -2.18607100 & 0.03732500 & -0.83869400 \\ 0 & -2.40112400 & 2.76733900 & -0.49174200 \\ \mathrm{H} & -2.53996300 & 3.07875600 & -1.39523700 \\ 0 & -0.02532500 & -0.27406800 & 3.34662500 \\ \mathrm{H} & -0.82501600 & -0.29007100 & 3.88768700 \\ 0 & -2.24270900 & -2.70942800 & -1.07978000\end{array}$


$\mathrm{H}$

C

$\mathrm{H}$

$\mathrm{H}$

C

$\mathrm{H}$

H

${ }^{1} \mathrm{~A} 2$-5TS

V

0

0

0

0

$\mathrm{Si}$

$\mathrm{Si}$

Si

0

0

0

0

$\mathrm{H}$

0

$\mathrm{H}$

0

$\mathrm{H}$

C

$\mathrm{H}$

$\mathrm{H}$

C

$\mathrm{H}$

$\mathrm{H}$

${ }^{1} \mathrm{~A} 3$

V

0

0

0

0

$\mathrm{Si}$

$\mathrm{Si}$

Si

0

0

$$
\begin{array}{r}
-3.12213400 \\
4.05329700 \\
4.69480300 \\
3.41193400 \\
3.91094200 \\
3.46091700 \\
4.72229700
\end{array}
$$

$-2.83915200$

$-0.56701700$

$-1.33665200$

$-0.79778500$

0.70658600

1.52316900

1.05319100

$-0.70214700$

0.37059900

$-0.06138800$

1.22134600

$-0.29536400$

0.28744500

$-0.95009700$
$-1.26654200$

$-2.93228200$

$-0.92883300$

$-0.13909700$

$-0.54928300$

0.67514100

1.45496900

1.06700000

1.22713300

1.50870500

1.90947400

1.55685300

2.50440600

0.92743700

1.84045300

2.43925100

2.19576600

$-3.94223700$

$-4.89692000$

$-4.28045200$

$-3.69488500$

$-2.83574600$

$-4.48869500$

0.35062300

1.21960300

$-0.16990400$

1.67262600

$-1.07134100$

$-0.36857400$

1.40159100

$-1.38529500$

$-1.61224100$

0.98956900

0.01784900

$-2.65948900$

$-2.84590300$

$-0.66397100$

$-0.62540900$

2.67273800

3. 24487100

0.41284200

0.83232000

$-0.24791100$

$-1.00016700$

$-1.47750800$

$-1.65245200$

$-0.37554400$

0.00749600

1.37369700

$-0.85568600$

$-1.19681800$

1.71335500

$-0.43028100$

$-0.91978000$

0.72480200

1.18126800

$-1.26259400$

$-1.81554200$

$-1.83338900$

3.30107400

3.61325600

$-0.72083900$

$-1.45969700$

$-0.12447400$

$-0.48401000$

0.87676000

$-0.30231400$

0.18200500

$-0.67766100$

0.92913300

$-1.10180000$

$-0.16714500$

3.69351500

0.81520200

0.10054400

1.27685300

0.76134900

$-0.55895600$

0.27382700

$-1.11124700$

1.48700100

$-0.39661400$

$-1.47025200$

$-1.30877800$

$-0.07872800$

1.67853600

$-0.33579400$

$-1.09853400$

$-0.14668600$

1.64892400

$-1.75619200$

$-0.50315100$

$-1.11752200$

$-1.27903100$

1.07061400

$-1.33761700$

$-0.63792800$

1.39681500

1.22283300 


$\begin{array}{rrr}-2.18833400 & -0.65145000 & 0.49491900 \\ -2.98013500 & -0.85617700 & -2.13672900 \\ -3.35910100 & -1.74315400 & -2.08945900 \\ 0.23062800 & 3.25860500 & -0.61341900 \\ -0.50981300 & 3.87567800 & -0.55251100 \\ -1.79249700 & -0.24511300 & 3.12270500 \\ -1.22902200 & -0.08428600 & 3.89035300 \\ 3.77941400 & -0.48813200 & 0.19486100 \\ 4.38953600 & -0.82613100 & 1.04622300 \\ 2.81962000 & 1.03862500 & -0.36443900 \\ 3.16981200 & -1.42482700 & -0.61233400 \\ 2.83864700 & -1.11712900 & -1.61668000 \\ 3.45079000 & -2.47298900 & -0.48283200\end{array}$

${ }^{1} \mathrm{~A} 4$

V

0

0

0

0

Si

Si

Si

0

0

0

0

$\mathrm{H}$

0

$\mathrm{H}$

0

$\mathrm{H}$

C

$\mathrm{H}$

$\mathrm{H}$

C

$\mathrm{H}$

$\mathrm{H}$

$-0.81853000$

$-0.00973100$

$-1.20676700$

$-2.95017900$

0.25446700

$-1.02826900$

$-1.00489600$

$-1.36498300$

0.01825000

$-0.65117700$

1.62785900

$-0.41945600$

0.80939600

$-0.30952700$

$-1.90719700$

0.19992600

$-1.27096000$

1.15552200

0.53668600

1.57581300

0.73704300

2.04847000

$-0.31106400$

$-0.78333900$

1.67189700

$-1.44734200$

0.37510100

0.20525400

0.29146600

1.76791900

1.97987600

1.17282000

$-0.01487600$

3.52188600

$-0.64419900$

$-1.40737500$

3.75338500

$-0.20432100$

$-2.23521900$

$-0.02294500$

$-2.39542000$

2. 32344400

0.69259900

$-2.50809500$

2. 96213300

0.62837800

3.00035100

1.53762600

1.34559200

3.09429200

2.17737500

$-3.50172800$

0.52434400

0.28423300

$-4.17622500$

1.38646600

0.30684900

$-2.77114700$

0.45751100

1.09662000

$-3.87572200$

$-0.69310100$

$-0.43921500$

$-3.40874800$

$-1.63952200$

$-0.14871400$

$-4.83566900$

$-0.74695600$

$-0.96401400$

${ }^{1} \mathrm{~A} 5$

$\mathrm{V}$

1.05916400
2.85575700
1.09644000
-0.24829100

$-0.55363700$

$-0.72958500$

$-1.11472400$

$-0.46967100$

0

$-0.11961900$

1.12075600

0

$-1.76063700$

$-0.87063900$ 
0

Si

Si

Si

0

0

0

0

$\mathrm{H}$

0

$\mathrm{H}$

0

$\mathrm{H}$

C

$\mathrm{H}$

$\mathrm{H}$

C

$\mathrm{H}$

$\mathrm{H}$

${ }^{1}$ B1

0

0

0

0

Si

Si

0

0

$\mathrm{H}$

0

$\mathrm{H}$

0

0

0

0

0

0

Si

0

$\mathrm{H}$

0

$\mathrm{H}$

V
0.44420300

$-0.36083700$

$-1.71920800$

$-1.04727400$

$-0.89379000$

$-1.49023200$

$-2.09432000$

$-1.53435900$

$-2.42062500$

$-0.30763400$

$-1.14477200$

$-2.84643300$

$-3.73895900$

3.97327100

4. 39301700

4.79502200

4.22926900

3.34780300

5.12425500

$-2.72227700$

$-1.84581000$

$-0.00948800$

$-1.82539500$

$-1.56042600$

$-1.52040200$

$-1.67304900$

$-2.65866300$

$-2.59197000$

$-2.57830400$

$-2.44289200$

2.70034200

1.78457100

1.87773900

1.70376300

$-0.02020900$

0.02708300

1.56244800

2.60519400

2.54498700

2.55098400

3.50307700

1.62979700
0.98746900

0.33494600

$-1.26021000$

1.47999300

1.67239300

$-0.86320000$

0.18744400

2.82352200

3.15495300

0.64486500

0.85420900

$-2.41631600$

$-2.24005000$

$-0.66657200$

1.22996100

$-1.39418600$

0.79244100

1.28444300

0.99333100

2.84351800

0.65440200

2.39807600

0.60232500

$-0.97827600$

$-1.04317500$

$-1.64223400$

$-1.57791100$

$-2.50837100$

$-1.80976700$

$-1.74716000$

2.86017100

0.65183300

0.62522500

$-1.62934300$

$-1.21100700$

$-1.22067400$

$-1.01380700$

$-1.71551200$

$-1.53464900$

$-1.74115400$

$-1.69687100$

1.72824700
$-1.38382300$

1.71794900

$-0.25024000$

$-0.80726200$

0.83741500

1.35006000

$-1.01122000$

$-1.59685200$

$-1.40393000$

3. 32450600

3.75716800

$-0.49194300$

$-0.16837800$

$-0.15507300$

$-1.02755500$

$-0.02823700$

$-0.02256900$

0.41891000

0.58334100

$-0.07981100$

1.34995400

$-0.05000400$

$-1.39562000$

1.52943200

$-1.51300900$

0.01572500

2.57167400

2.82231400

$-2.48499600$

$-3.43950100$

0.02669300

1.38975300

$-1.35079800$

0.02429600

2.07242200

$-2.04667300$

$-1.50752100$

2.52195900

3.46904000

$-2.57805100$

$-2.42089600$

0.00280900 
1B2

0

0

0

0

Si

Si

0

0

$\mathrm{H}$

0

$\mathrm{H}$

0

0

0

0

0

Si

0

$\mathrm{H}$

0

$\mathrm{H}$

V

V

Si

0

C

$\mathrm{H}$

$\mathrm{H}$

C

$\mathrm{H}$

$\mathrm{H}$

${ }^{1} \mathrm{~B} 2$ - 3 TS

0

0

0

Si

Si

0

0

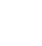

0
si
0
0

$-1.64753400$

1.71699900

$-0.04494900$

1.52287300

$-0.99739900$

1.53724700

1.27522900

3.70372200

0.10530600

1.58912400

1.36910500

$-1.32098800$

$-0.91771500$

2.08621100

0.00243900

1.46718500

1.29376600

1.42089200

2.20068400

$-0.17126700$

$-1.48140900$

2.05500200

$-0.26850800$

1.55503600

2.57610600

$-0.68902100$

0.04906400

3.50488300

$-0.10539700$

$-2.45963600$

3.92941200

$-0.93972900$

$-2.69731800$

3.30989800

$-0.37793300$

2. 59101700

3.10804700

$-0.38761400$

3.53559500

$-1.29267800$

$-0.22548900$

$-1.28151600$

$-1.70302600$

$-0.58159200$

1.28732400

$-0.37206500$

$-2.40540800$

$-0.10760100$

1.05246100

$-1.19072900$

$-2.08724200$

0.80902600

$-1.23586800$

2.02693400

$-0.60050200$

$-1.82964500$

1.42676000

$-1.16235500$

$-2.63246900$

$-2.63707900$

$-0.99113600$

$-2.46294300$

$-3.57221700$

$-1.10468500$

$-2.98676000$

2.46151300

$-1.89267900$

$-3.48947500$

2.21916700

$-2.01477300$

0.69173700

0.10520200

0.84374200

2.20671100

0.04650100

$-0.45738900$

$-1.61891800$

$-1.56268400$

$-3.40843800$

1.48584800

0.90363700

$-4.48878800$

1.30967100

$-0.01089300$

$-5.29540200$

0.72348700

0.46368800

$-4.89597400$

2.29130500

$-0.31089700$

$-3.73179700$

0.57933700

$-1.08915400$

$-3.49849900$

1.11853600

$-2.01714200$

$-3.95928000$

$-0.47905800$

$-1.27011000$

-1.49727200
0.77462400
-1.79693500
-1.92681700
-2.29633900
-2.54941200
-2.97875300

1.49574000

1. 31261900

1.95300100

$-0.37604700$

1.13612100

$-1.41266200$

$-0.06267800$

1.69500700

$-0.44410300$

$-1.30568800$

$-0.73047800$

0.31205600

$-0.12633800$

2.94668000 


\begin{tabular}{|c|c|c|c|}
\hline $\mathrm{H}$ & -3.71545000 & 0.49743200 & 2.92845400 \\
\hline 0 & -3.63666600 & -0.73655100 & -2.19793200 \\
\hline $\mathrm{H}$ & -4.34389800 & -0.08143200 & -2.14680000 \\
\hline 0 & 1.86045400 & -0.40161600 & 1.17638300 \\
\hline 0 & 1.32182800 & -0.40371500 & -1.39406900 \\
\hline 0 & 0.44277500 & -2.36546400 & 0.11979000 \\
\hline 0 & -0.60522800 & -0.92617400 & 2.16476700 \\
\hline 0 & -1.13360600 & -1.46567300 & -1.86169300 \\
\hline Si & 0.43082300 & -1.78065800 & -1.43925200 \\
\hline 0 & 1.41033400 & -2.63559900 & 2.63454200 \\
\hline $\mathrm{H}$ & 0.95037400 & -2.70841300 & 3.48067900 \\
\hline 0 & 1.06532900 & -2.86748300 & -2.48695100 \\
\hline $\mathrm{H}$ & 0.43124500 & -3.38294000 & -3.00159100 \\
\hline Si & 0.75636400 & -1.60707500 & 1.54552500 \\
\hline V & 2.11087100 & 0.68916700 & -0.17958800 \\
\hline 0 & 3.14879800 & 2.18188700 & 0.46403400 \\
\hline C & 4.13977800 & 1.00773000 & 0.27712000 \\
\hline $\mathrm{H}$ & 4.38700400 & 0.46830500 & 1.19522800 \\
\hline $\mathrm{H}$ & 4.35696500 & 2.24565900 & 0.32548100 \\
\hline C & 3.96646200 & 0.49322700 & -1.05703800 \\
\hline $\mathrm{H}$ & 4.05655600 & 1.17883200 & -1.91032200 \\
\hline $\mathrm{H}$ & 4.26938700 & -0.54213600 & -1.24682200 \\
\hline 0 & -1.30978700 & 3.66996800 & -0.42330300 \\
\hline V & -0.92208400 & 2.16392600 & -0.24253300 \\
\hline \multicolumn{4}{|c|}{${ }^{1} \mathrm{~B} 2-4 \mathrm{TS}$} \\
\hline V & -1.72243800 & 0.65273900 & -0.85644500 \\
\hline 0 & -3.47253000 & 1.27210400 & -0.88701900 \\
\hline C & -4.46670800 & 1.58326200 & 0.71057900 \\
\hline $\mathrm{H}$ & -4.87203400 & 2.59074300 & 0.60360900 \\
\hline $\mathrm{H}$ & -3.80497300 & 1.34272700 & 1.54335200 \\
\hline $\mathrm{C}$ & -4.65140000 & 0.60984500 & -0.34342800 \\
\hline $\mathrm{H}$ & -4.45796000 & -0.44067400 & -0.08622400 \\
\hline $\mathrm{H}$ & -5.50538200 & 0.73274600 & -1.02302700 \\
\hline 0 & -1.96566600 & -0.07651500 & 0.83107000 \\
\hline 0 & -0.59316000 & 2.02499200 & -0.97024600 \\
\hline 0 & -1.17985200 & -0.80586700 & -1.68431600 \\
\hline $\mathrm{Si}$ & -1.05858500 & -1.20896700 & 1.58841400 \\
\hline Si & -0.23761000 & -2.09328900 & -1.18952500 \\
\hline 0 & -0.49377100 & -2.26443300 & 0.42719300 \\
\hline 0 & -1.97980200 & -1.98162500 & 2.70402900 \\
\hline $\mathrm{H}$ & -1.51922000 & -2.58113600 & 3.30464400 \\
\hline 0 & -0.62849700 & -3.48250900 & -1.95836900 \\
\hline $\mathrm{H}$ & -0.09482100 & -3.71130100 & -2.73003400 \\
\hline
\end{tabular}




$\begin{array}{lrrr}0 & 1.64238900 & 3.58611200 & -0.77637300 \\ 0 & 1.28698400 & 1.66142300 & 1.19066500 \\ 0 & 2.06636400 & 0.90939100 & -1.34209000 \\ 0 & 2.45444500 & -0.68961100 & 0.73415600 \\ 0 & 0.27171200 & -0.55272800 & 2.33443400 \\ \text { O } & 1.33726300 & -1.70586500 & -1.49602600 \\ \text { Si } & 2.46305900 & -0.65485900 & -0.92360400 \\ \text { O } & 2.68196400 & 0.35896700 & 3.16154500 \\ \text { H } & 2.31912000 & 0.76440200 & 3.95929600 \\ \text { O } & 3.89807600 & -1.12252800 & -1.54990600 \\ \text { H } & 4.66373800 & -0.56644300 & -1.35711100 \\ \text { V } & 1.09958300 & 2.14714400 & -0.50251800 \\ \text { Si } & 1.66874400 & 0.19015400 & 1.88819500\end{array}$

$\begin{array}{lrrr}{ }^{1} \text { B2-5TS } & & & \\ \text { V } & -1.77299700 & 0.77452000 & -0.69813800 \\ \text { O } & -3.42581200 & 1.53130000 & -0.86715800 \\ \text { C } & -4.60615400 & 1.47433200 & -0.24632600 \\ \text { H } & -5.41781400 & 1.99664900 & -0.78330300 \\ \text { H } & -4.59019300 & 1.99601300 & 0.82122900 \\ \text { C } & -4.99872700 & 0.41706800 & 0.64689000 \\ \text { H } & -4.22970000 & -0.18811200 & 1.13510500 \\ \text { H } & -6.04850500 & 0.33357900 & 0.93935800 \\ \text { O } & 1.64993800 & 3.67296600 & -0.35036500 \\ \text { O } & 2.12751900 & 1.05730200 & -1.23926200 \\ \text { O } & -0.53569700 & 2.07384600 & -0.65949800 \\ \text { O } & 1.38061600 & 1.50911400 & 1.38899500 \\ \text { Si } & 2.48676900 & -0.54721100 & -1.00103800 \\ \text { Si } & 1.68971500 & -0.04257500 & 1.90113600 \\ \text { O } & 2.47025800 & -0.78763400 & 0.64147600 \\ \text { O } & 3.92433300 & -0.95474400 & -1.67141500 \\ \text { H } & 4.63016500 & -0.29957200 & -1.60425200 \\ \text { O } & 2.57534000 & -0.07580800 & 3.27849900 \\ \text { H } & 3.36478700 & 0.47943000 & 3.29823000 \\ \text { O } & -1.15576400 & -0.54952500 & -1.71771700 \\ \text { O } & -1.96094700 & -0.20839400 & 0.82233700 \\ \text { O } & -0.49005700 & -2.31073300 & 0.14266000 \\ \text { O } & 1.35466300 & -1.52292700 & -1.68768100 \\ 0 & 0.29069100 & -0.83524200 & 2.23650800 \\ \text { Si } & -1.02976500 & -1.42145000 & 1.43644400 \\ 0 & -0.64924600 & -3.15476500 & -2.40316900 \\ \text { H } & -0.05363200 & -3.33604800 & -3.14118000 \\ \text { O } & -1.94928600 & -2.31202500 & 2.46066300 \\ \text { H } & -1.47583400 & -2.83738400 & 3.11825100\end{array}$


${ }^{1}$ B3

0

0

0

Si

Si

0

0

$\mathrm{H}$

0

$\mathrm{H}$

0

0

0

0

0

Si

0

$\mathrm{H}$

0

$\mathrm{H}$

Si

V

0

C

$\mathrm{H}$

$\mathrm{H}$

C

$\mathrm{H}$

$\mathrm{H}$

0

v

${ }^{1}$ B4

V

0

C

$\mathrm{H}$

$\mathrm{H}$

C

$\mathrm{H}$
$-1.45222300$

0.89284400

$-1.62170500$

$-1.95193900$

$-2.25005700$

$-2.56367100$

$-3.05445200$

$-3.85489000$

$-3.65266400$

$-3.60860700$

1.75027000

1.48100600

0.34483300

$-0.67783900$

$-1.11251400$

0.38826100

1.45400900

0.86046000

0.87775000

0.26416900

0.73990900

1.94844800

3.81155300

3.94105100

4. 36102400

3.44888900

3.83497900

3.77136900

4.21667500

$-1.26764600$

$-0.85097400$

$-1.62632900$

$-3.55207600$

$-3.77476600$

$-4.18036200$

$-2.96090400$

$-4.57426500$

$-4.33120600$
1.64206000

1.99162600

1.03105200

0.13448300

$-0.49832600$

$-0.62185900$

0.24159500

$-0.73205400$

$-0.78239200$

$-0.18957800$

$-0.85707500$

$-2.32358700$

$-0.70871000$

$-1.59851500$

$-2.08333000$

$-2.20623200$

$-2.59776500$

$-3.39709200$

$-4.14014200$

$-1.34413800$

0.55608600

1.70940200

0.77969100

$-0.19923100$

1.25707500

1.19219500

2.26021500

0.53127000

3.65617300

2. 18603200
0.75163600

1.16897400

$-0.50876400$

$-1.50271300$

1.70156900

$-1.25728800$

0.35608300

2.90150800

2.72307100

$-2.06209200$

$-3.02553300$

1.14420100

$-1.50726000$

0.40628300

2.28516500

$-1.71943400$

$-1.22284700$

2.89878900

3. 55212600

$-2.06214100$

$-2.12491100$

1.70033300

$-0.57164000$

1.05302500

0.06363500

0.33658900

1.83465300

$-1.27482200$

$-1.51697800$

$-2.06015100$

$-0.72276700$

$-0.40172300$

$-0.08517200$

$-1.16789000$

0.79545000

$-0.90468200$

1.59986100

0.28321400

2.59338300

0.06734700

1.55325300

1.01552300

0.39594700

0.03899300

$-0.51783800$

0.58998900 
H

0

0

0

Si

Si

0

0

$\mathrm{H}$

0

$\mathrm{H}$

0

0

0

0

0

0

Si

0

$\mathrm{H}$

0

$\mathrm{H}$

V

Si

${ }^{1}$ B5

$\mathrm{V}$

0

C

$\mathrm{H}$

$\mathrm{H}$

C

$\mathrm{H}$

$\mathrm{H}$

0

0

0

0

Si

Si

0

0

$\mathrm{H}$

0
$-5.58950300$

$-1.96166000$

$-1.05186900$

$-0.47282900$

$-0.87325400$

0.71014400

0.21789000

$-1.67912300$

$-1.14513300$

0.93531300

1.54257400

0.42758700

0.25748300

1.83535000

2.30937300

$-0.03802000$

2.11057400

2.65101400

1.61248300

0.97569700

4.25701300

4.71314100

0.33003700

1.02814500

$-1.59726800$

$-3.29397000$

$-4.45795000$

$-5.14904100$

$-5.94992000$

$-4.95256300$

$-4.24806700$

$-5.06256700$

2. 26282000

1.72940800

$-0.24841000$

2.07291800

1.88520700

2.23919900

2.40301500

3.01685600

2.77813600

3.50177600
0.48657700

$-0.91069000$

1.60594700

$-1.23407500$

$-1.69551300$

$-2.17080300$

$-2.47011600$

$-2.75198200$

$-3.33311400$

$-3.60419200$

$-3.60533200$

3.75989700

1.79555600

1.41219200

0.09106100

$-0.60509900$

$-1.29707000$

0.09942900

1.20061100

1.34082300

0.17036000

0.98098600

2. 21167000

0.62075500

1.14568300

1.89880300

1.55415900

2. 34591400

0.13968300

0.15874400

$-0.46529600$

$-0.27447000$

3.39364700

1. 30863700

2.30965700

0.79489700

$-0.29344500$

$-0.85650200$

$-1.11457600$

$-0.48328600$

$-0.19333600$

$-1.44580400$
$-0.36131300$

0.41993800

$-1.48646300$

$-1.86318000$

1.37544300

$-1.15706200$

0.39050200

2. 33460300

2.89105900

$-1.91264500$

$-2.66351100$

$-0.79973800$

1.12869300

$-1.11103800$

1.13475100

2. 30252900

$-1.16357600$

$-0.48819800$

3.44368800

4.15554100

$-0.78448400$

$-0.52486700$

$-0.60435700$

2.02892200

0.55656400

0.22552100

$-0.04732200$

$-0.38909200$

0.54502800

0.07498100

0.64339400

$-0.93614000$

0.44887000

$-1.29698300$

0.56174400

1.37935900

$-1.76073600$

1.20448100

$-0.42404500$

$-2.92700000$

$-3.81663100$

2.05859500 


$\begin{array}{lrrr}\text { H } & 4.36756500 & -1.05330100 & 1.88817700 \\ 0 & -1.80305000 & 0.25044900 & -1.07807900 \\ 0 & -1.42054700 & -0.28953500 & 1.57745000 \\ 0 & -0.83610200 & -2.10687000 & -0.26939600 \\ \text { O } & 0.42034600 & -0.84075700 & -2.25767000 \\ \text { O } & 0.89850900 & -1.62645900 & 1.75720200 \\ \text { Si } & -0.69256600 & -1.77063000 & 1.33322100 \\ \text { O } & -2.02869400 & -1.86087300 & -2.70821500 \\ \text { H } & -1.63243700 & -2.60767100 & -3.17444500 \\ \text { O } & -1.39007600 & -2.95346100 & 2.22371700 \\ \text { H } & -0.92106100 & -3.21482900 & 3.02636800 \\ \text { V } & 1.47085500 & 2.05720700 & 0.28281100 \\ \text { Si } & -1.08115700 & -1.11487600 & -1.59316600\end{array}$

${ }^{1} \mathrm{C} 1$

0

0

0

Si

Si

0

0

$\mathrm{H}$

0

$\mathrm{H}$

0

0

0

0

0

0

Si

0

$\mathrm{H}$

0

$\mathrm{H}$

V

Si

Si

0

$\mathrm{H}$

${ }^{1} \mathrm{C} 2$

0
$-1.77335800$

$-0.09776300$

$-1.78481100$

$-1.60506500$

$-1.58675000$

$-1.76495900$

$-2.72793600$

$-2.63026000$

$-2.67819200$

$-2.41958300$

2. 59505700

1.72834800

1.80144400

1.64135000

$-0.08319900$

$-0.05491500$

1.48247000

2.53999600

2.45143400

2.46708100

3.41745300

1.54723300

1.45844900

$-1.62961500$

$-2.75390500$

$-2.51214100$

$-1.48407900$
0.82863200

2.37645200

0.66661800

$-0.79055100$

$-0.97808100$

$-1.54319100$

$-1.25532000$

$-2.12830400$

$-1.69300700$

$-1.84123000$

2.89094800

0.70963100

0.58634200

$-1.61816000$

$-1.11631500$

$-1.28530300$

$-1.05786500$

$-1.61707600$

$-1.43030600$

$-1.82551100$

$-1.79483000$

1.73705800

$-0.93183100$

1.72039400

2.89509000

3.77477400

1.27598400

$-0.12894500$

$-1.37484800$

1.55107900

$-1.45965500$

0.08244300

2.63847000

3.03939700

$-2.43671900$

$-3.35522000$

$-0.08983900$

1.35459500

$-1.38021700$

0.07717500

2.11457500

$-1.99578700$

$-1.47523400$

2.57764600

3.52117400

$-2.52329900$

$-2.35381600$

$-0.06674300$

1.56661200

$-0.11442000$

$-0.23561100$

0.08122000 
0

0

Si

Si

0

0

$\mathrm{H}$

0

$\mathrm{H}$

0

0

0

0

0

Si

0

$\mathrm{H}$

0

$\mathrm{H}$

V

Si

0

C

$\mathrm{H}$

$\mathrm{H}$

C

$\mathrm{H}$

$\mathrm{H}$

0

$\mathrm{H}$

Si

${ }^{1} \mathrm{C} 2-3 T S$

0

0

0

Si

Si

0

0

$\mathrm{H}$

0

$\mathrm{H}$

0
0.84910100

$-1.34614800$

$-2.19723600$

$-2.02913200$

$-2.61416300$

$-3.48495300$

$-3.85537500$

$-3.21431700$

$-3.12519200$

1.26108200

1.64663000

0.28197500

$-1.12652500$

$-0.87576300$

0.52073300

1.02717200

0.85577800

1.00154000

1.77210600

1.95453500

0.37324000

3.33939600

4.41657400

5.23726800

4.80681300

3.66879000

3.42649600

3.91691500

$-1.24095700$

$-0.68520200$

$-0.80874900$

$-1.31515700$

0.78279700

$-1.66894000$

$-1.88111100$

$-2.33929500$

$-2.59869500$

$-2.90040100$

$-3.66496900$

$-3.77430700$

$-3.75362800$

1.82628000
2.07109500

1.37601300

0.00723900

$-0.12187700$

$-0.52003400$

0.17631800

$-0.62959400$

$-0.10293900$

$-0.73189900$

$-0.21719700$

$-0.60352400$

$-2.38754000$

$-1.08333900$

$-1.21715100$

$-1.83122300$

$-2.59380600$

$-2.39111700$

$-3.01507200$

$-3.53500600$

0.69567400

$-1.57499100$

1.48449400

1.36455600

0.77356400

2.36465500

0.66791100

1.24081100

$-0.37698000$

3.78326500

4.38391600

2.20765100

$-0.03081800$

$-1.39293100$

1.45158200

$-1.56085700$

$-0.06492600$

2.44180200

2.82316200

$-2.68383300$

$-3.41090400$

1.29437700

$-1.26855700$

0.14633400

2.08420100

$-2.00434700$

$-1.39478800$

2.69039200

3.61879300

$-2.41276000$

$-2.15152100$

$-0.10976000$

1.58729700

$-0.93927000$

$-0.01434500$

$-0.45872900$

0.24657200

1.09269500

1.99786300

1.31905100

$-0.07532100$

$-0.58818800$

$-0.06180300$
1.61588700

1.99283600

1.19518700

0.15079500

$-0.30696300$

$-0.55352600$

0.27984600

0.85808800

$-0.44873400$

$-0.45467800$

$-0.40132700$
1.18391600

$-0.45285300$

$-1.40512600$

1.69638700

$-1.23820900$

0.37457500

2.96743400

2.85233900

$-2.01144900$

$-2.97650600$

1.15420500 


$\begin{array}{lrrr}\text { O } & 1.22087400 & -0.42890500 & -1.38682800 \\ \text { O } & 0.31596200 & -2.34100200 & 0.17428200 \\ \text { O } & -0.63389900 & -0.79725300 & 2.19747800 \\ \text { O } & -1.26647100 & -1.43017500 & -1.81070200 \\ \text { Si } & 0.29497900 & -1.78143700 & -1.39439900 \\ \text { O } & 1.24935500 & -2.56974100 & 2.72317400 \\ \text { H } & 1.85048600 & -3.26340800 & 2.42432500 \\ \text { O } & 0.89014200 & -2.90695300 & -2.42825900 \\ \text { H } & 0.23566100 & -3.44600900 & -2.89006900 \\ \text { Si } & 0.68247200 & -1.54816800 & 1.57831000 \\ \text { V } & 2.04464200 & 0.69344000 & -0.21992600 \\ \text { O } & 3.12488800 & 2.14564700 & 0.48873400 \\ \text { C } & 4.08969000 & 0.96549700 & 0.19088100 \\ \text { H } & 4.34934800 & 0.36117800 & 1.06332800 \\ \text { H } & 4.32322300 & 2.19343800 & 0.31196800 \\ \text { C } & 3.85466800 & 0.53616100 & -1.16933500 \\ \text { H } & 3.95306600 & 1.26758100 & -1.98273500 \\ \text { H } & 4.13150100 & -0.49156900 & -1.42859900 \\ \text { Si } & -0.83902700 & 2.14497500 & -0.32524800 \\ \text { O } & -1.29624600 & 3.70159900 & -0.58104600 \\ \text { H } & -0.61929700 & 4.36037400 & -0.38106500\end{array}$

${ }^{1} \mathrm{C} 2-4 T S$
$\mathrm{~V}$
$\mathrm{O}$
$\mathrm{C}$
$\mathrm{H}$
$\mathrm{H}$
$\mathrm{C}$
$\mathrm{H}$
$\mathrm{H}$
$\mathrm{O}$
$\mathrm{O}$
$\mathrm{O}$
$\mathrm{Si}$
$\mathrm{Si}$
$\mathrm{O}$
$\mathrm{O}$
$\mathrm{H}$
$\mathrm{O}$
$\mathrm{H}$
$\mathrm{O}$
$\mathrm{O}$
$\mathrm{O}$

$-1.65016300$

0.54828100

$-0.94478500$

$-3.39794100$

1.20847000

$-1.00772100$

$-4.40099000$

1.64423900

0.54583400

$-4.78397900$

2.64986200

0.36538000

$-3.75480900$

1.44711300

1.40152000

$-4.59422800$

0.60572400

$-0.44162400$

$-4.42491000$

$-0.42825900$

$-0.11070400$

$-5.43868200$

0.69789000

$-1.13853200$

$-1.96061700$

$-0.05595600$

0.78590700

$-0.52746900$

1.91523500

$-1.14675100$

$-1.08319200$

$-0.97270000$

$-1.65454600$

$-1.01300600$

$-1.08154200$

1.64003900

$-0.09129900$

$-2.15971000$

$-1.03330000$

$-0.37822800$

$-2.20980000$

0.58860600

$-1.91038000$

$-1.80409300$

2.80945200

$-1.44553900$

$-2.42026400$

3.38940000

$-0.38195100$

$-3.63267100$

$-1.68574100$

0.19069900

$-3.89313500$

$-2.41828700$

1.17364900

1.85073900

0.97078300

1.97518600

0.94995500

$-1.39042600$

2.50421400

$-0.43583300$

0.79885700 


$\begin{array}{lrrr}0 & 0.27353800 & -0.29351700 & 2.33628000 \\ \text { O } & 1.47283100 & -1.71964400 & -1.33417800 \\ \text { Si } & 2.50213000 & -0.52979600 & -0.85747300 \\ \text { O } & 2.55544600 & 0.89125600 & 3.12041700 \\ \text { H } & 2.09662100 & 1.01946500 & 3.95987800 \\ \text { O } & 3.96813000 & -0.91873300 & -1.46830600 \\ \text { H } & 4.68489200 & -0.29179600 & -1.30851100 \\ \text { Si } & 1.62146300 & 0.50482300 & 1.83339700 \\ \text { O } & 1.57364300 & 3.62148300 & -1.01061700 \\ \text { H } & 1.08382300 & 4.09469700 & -1.69481700 \\ \text { Si } & 1.04228900 & 2.11341600 & -0.65670900\end{array}$

$\begin{array}{lrrr}{ }^{1} \mathrm{C} 2-5 T S & & & \\ \mathrm{~V} & -1.64997400 & 0.66741300 & -0.86826500 \\ \mathrm{O} & -3.30303200 & 1.44225500 & -1.03460400 \\ \mathrm{C} & -4.47206000 & 1.48794600 & -0.39755800 \\ \mathrm{H} & -5.27416700 & 1.97738000 & -0.97957000 \\ \mathrm{H} & -4.42382200 & 2.11788200 & 0.60322200 \\ \mathrm{C} & -4.90127400 & 0.53118600 & 0.59333200 \\ \mathrm{H} & -4.15413800 & -0.03931600 & 1.14877700 \\ \mathrm{H} & -5.95612200 & 0.50511900 & 0.87819000 \\ \mathrm{O} & 2.07857400 & 1.15123900 & -1.18944500 \\ \mathrm{O} & -0.47774500 & 2.03306400 & -1.01216000 \\ \mathrm{O} & 1.05113300 & 1.69573400 & 1.19088200 \\ \mathrm{Si} & 2.58159400 & -0.37325400 & -0.80906900 \\ \mathrm{Si} & 1.51069700 & 0.28779600 & 1.92785600 \\ \mathrm{O} & 2.46896500 & -0.50974900 & 0.84193800 \\ \mathrm{O} & 4.11688700 & -0.55976300 & -1.34464900 \\ \mathrm{H} & 4.40890900 & -1.46667400 & -1.49995600 \\ \mathrm{O} & 2.34949100 & 0.57615100 & 3.30502100 \\ \mathrm{H} & 1.88034600 & 0.39668300 & 4.12903300 \\ \mathrm{O} & -0.92299300 & -0.69813600 & -1.78019300 \\ \mathrm{O} & -1.97933100 & -0.25339300 & 0.67637500 \\ \mathrm{O} & -0.31388500 & -2.30219800 & 0.26561300 \\ \mathrm{O} & 1.61006800 & -1.52605200 & -1.48188300 \\ \mathrm{O} & 0.18078300 & -0.62085800 & 2.29038600 \\ \mathrm{Si} & -1.03017200 & -1.36137400 & 1.43264800 \\ \mathrm{O} & -0.25891700 & -3.34530200 & -2.17557200 \\ \mathrm{H} & 0.00331700 & -3.33208800 & -3.10468800 \\ \mathrm{O} & -1.97148800 & -2.24661700 & 2.44412100 \\ \mathrm{H} & -1.53145900 & -2.93227200 & 2.96204700 \\ \mathrm{Si} & 0.02271300 & -1.97481800 & -1.32056500 \\ \mathrm{Si} & 1.03692700 & 2.18619500 & -0.40265900 \\ \mathrm{O} & 1.50912400 & 3.74621100 & -0.56799300\end{array}$


${ }^{1} \mathrm{C} 3$

0

0

0

Si

Si

0

0

$\mathrm{H}$

0

$\mathrm{H}$

0

0

0

0

0

Si

0

$\mathrm{H}$

0

$\mathrm{H}$

Si

V

0

C

$\mathrm{H}$

$\mathrm{H}$

C

$\mathrm{H}$

$\mathrm{H}$

Si

0

$\mathrm{H}$

${ }^{1} \mathrm{C} 4$

$\mathrm{V}$

0

C

$\mathrm{H}$

$\mathrm{H}$

C

$\mathrm{H}$

$\begin{array}{rrr}1.34660700 & -1.78344400 & 1.02351900 \\ -0.82660600 & -1.98607200 & -0.54779700 \\ 1.51794700 & -1.04560000 & -1.52032100 \\ 1.92904300 & -0.36421100 & 1.66126600 \\ 2.25690300 & 0.41280800 & -1.23109800 \\ 2.60690700 & 0.45134500 & 0.38070600 \\ 3.00438400 & -0.62571700 & 2.86416200 \\ 3.78619600 & -1.14802500 & 2.64447900 \\ 3.64886400 & 0.58772100 & -2.07000400 \\ 3.57329800 & 0.86863700 & -2.99056600 \\ -1.73801300 & 0.12653100 & 1.14339800 \\ -1.40542900 & 0.90904200 & -1.48581600 \\ -0.27556500 & 2.26261700 & 0.51405900 \\ 0.70789000 & 0.52254300 & 2.29794100 \\ 1.19921000 & 1.61744100 & -1.62792100 \\ -0.29957800 & 2.10388100 & -1.12581700 \\ -1.38217900 & 2.04377700 & 2.99943000 \\ -0.77277500 & 2.38548600 & 3.66588500 \\ -0.75660300 & 3.46715000 & -1.90426400 \\ -0.14646000 & 4.21530300 & -1.88288700 \\ -0.69545100 & 1.22364900 & 1.75506800 \\ -1.86770500 & -0.54775400 & -0.61485500 \\ -3.84309700 & -1.70528800 & 0.94898700 \\ -3.87671100 & -0.79004300 & -0.06314500 \\ -4.30343700 & 0.19882000 & 0.16485000 \\ -3.45762800 & -1.25856900 & 1.72348200 \\ -3.70812700 & -1.21688200 & -1.39356800 \\ -3.63220300 & -2.28720300 & -1.61961100 \\ -4.05903900 & -0.56846100 & -2.20367700 \\ 0.82290100 & -2.15328200 & -0.50455600 \\ 1.27090300 & -3.65527700 & -0.97392300 \\ 0.68310400 & -4.38009300 & -0.72534900\end{array}$

1.49309900

0.74404500

$-1.09229500$

3.59274100

0.50361500

$-0.64350600$

4. 38097100

$-0.69838900$

$-0.78831700$

5.41471400

$-0.50830600$

$-1.09679700$

3.85403400

$-1.51474800$

$-1.29197600$

3.92028800

$-0.27022100$

0.53621600

3.06533100

$-0.77848600$ 


\begin{tabular}{|c|c|c|c|}
\hline $\mathrm{H}$ & 4.60152400 & 0.24243200 & 1.22285700 \\
\hline 0 & 1.48381800 & 1.69101000 & 0.45867400 \\
\hline 0 & 1.46946500 & -1.05128000 & -1.33647900 \\
\hline 0 & 0.03898100 & 1.42130900 & -1.86945800 \\
\hline Si & 0.15154300 & 1.90969500 & 1.40853800 \\
\hline Si & -1.42171700 & 1.79286900 & -1.17178900 \\
\hline 0 & -1.12418500 & 2.23682300 & 0.39217300 \\
\hline 0 & 0.32667500 & 3.09343000 & 2.53057700 \\
\hline $\mathrm{H}$ & 0.42094000 & 3.99167900 & 2.18949000 \\
\hline 0 & -2.19685900 & 3.03174200 & -1.91380400 \\
\hline $\mathrm{H}$ & -2.76975600 & 2.79302400 & -2.65331600 \\
\hline 0 & 0.45184300 & -1.82375000 & 1.03849000 \\
\hline 0 & -1.07116500 & -1.94531600 & -1.16015200 \\
\hline 0 & -2.05682600 & -0.95821700 & 1.07936300 \\
\hline 0 & -0.15726700 & 0.52858100 & 2.26266100 \\
\hline 0 & -2.37315900 & 0.43974300 & -1.21121300 \\
\hline Si & -2.33783200 & -1.06295600 & -0.55180600 \\
\hline 0 & -0.84739100 & -1.81180000 & 3.37672900 \\
\hline $\mathrm{H}$ & -1.04304600 & -1.26680900 & 4.14950800 \\
\hline 0 & -3.78270200 & -1.75064300 & -0.89310700 \\
\hline $\mathrm{H}$ & -3.92249000 & -2.64735900 & -0.56373400 \\
\hline Si & -0.65556300 & -1.00897000 & 1.96382300 \\
\hline Si & 0.47736100 & -2.13549500 & -0.59173300 \\
\hline 0 & 1.00891000 & -3.67689100 & -0.78801800 \\
\hline $\mathrm{H}$ & 1.13377600 & -3.97164700 & -1.69902900 \\
\hline
\end{tabular}

1.10321700

0.66663800

1.91606600

0.35996300

1.61627800

0.06280500

2.44405700

$-0.22183700$

0.19272900

0.55519400

0.22420800

0.09187300

$-0.44703000$

0.62627100

$-0.14707900$

$-0.94345600$

1.55702200

$-1.02949700$

2. 23319000

0.75992900

0.72150600

1.47860700

0.06519900

$-1.72193600$

$-0.87518700$

1.12590700

$-0.95022800$

$-0.50843400$

0.19332100

$-2.91923500$

$-0.46684800$

$-3.62166100$

$-1.44011900$

1.88256300 


$\begin{array}{lrrr}H & 3.41951000 & -1.92611500 & 2.70320500 \\ 0 & -1.71290000 & 0.39030000 & -1.06723500 \\ 0 & -1.41911000 & -0.44148800 & 1.55039900 \\ 0 & -0.75367600 & -2.04285300 & -0.49146700 \\ 0 & 0.52168900 & -0.50430800 & -2.31619700 \\ \text { O } & 0.92465300 & -1.77597200 & 1.57839200 \\ \text { Si } & -0.67327800 & -1.86615600 & 1.14806100 \\ \text { O } & -1.83492200 & -1.66923500 & -2.88327300 \\ \text { H } & -1.86504700 & -1.20904300 & -3.73120500 \\ \text { O } & -1.41287900 & -3.09063000 & 1.94712200 \\ \text { H } & -1.06780400 & -3.97993200 & 1.79701800 \\ \text { Si } & -0.97033800 & -0.91854500 & -1.70076400 \\ \text { Si } & 1.46550900 & 2.00418500 & 0.53989300 \\ \text { O } & 2.21991800 & 3.39796200 & 0.94413200 \\ \text { H } & 3.17595800 & 3.43312300 & 0.81332300\end{array}$

${ }^{3} \mathrm{~A} 2$

V

0

0

0

0

Si

Si

Si

0

0

0

0

$\mathrm{H}$

0

$\mathrm{H}$

0

$\mathrm{H}$

C

$\mathrm{H}$

$\mathrm{H}$

C

$\mathrm{H}$

$\mathrm{H}$

$-1.24211500$

$-0.69732800$

$-0.08747100$

$-2.89386800$

$-1.24639400$

0.03000600

$-0.71952100$

0.46181500

$-1.36871500$

0.09962700

$-1.86364000$

$-0.21395200$

$-0.75869700$

0.23655900

1.40599500

0.80732700

1.12338100

$-1.32844500$

1.68084800

$-1.31309700$

$-0.11311600$

0.77407600

0.86691900

1.50810300

1.01206300

1.83523500

0.17031200

1.85385200

$-0.18647300$

$-1.33464100$

1.80603700

$-0.44238700$

1.30476900

1.07802400

1.72437500

2.86232900

0.84030500

1.31633500

3.70480900

1.03221300

2.15263100

$-2.57098900$

1.83117300

2.69543800

$-2.56469400$

2.71149700

$-2.57016500$

$-0.21206600$

3.65585900

$-2.38606500$

$-0.12657600$

$-3.90920000$

0.96282600

0.03733800

$-4.48107900$

1.66715800

$-0.57063800$

$-3.26301300$

1.34781300

0.83221800

$-4.10711300$

$-0.50437000$

$-0.10196600$

$-4.79335600$

$-0.88865300$

0.68335000

$-4.56469500$

$-0.75749700$

$-1.07483900$

${ }^{3} \mathrm{~A} 2-3 \mathrm{TS}$

$\mathrm{V}$

0

$-1.42918200$

0.09821900

0.01147500

$-3.13082700$

$-1.11118500$

$-0.14929500$ 


\section{${ }^{3} \mathrm{~A} 2-4 \mathrm{TS}$}

$\mathrm{V}$

0

0

0

0

Si

Si

Si

0

0

0

0

$\mathrm{H}$

0

$\mathrm{H}$

0

$\mathrm{H}$

C

$\mathrm{H}$

$\mathrm{H}$

C

$\mathrm{H}$
$-0.62356300$

$-0.66938800$

$-0.41982600$

1.01661000

1.01688800

1.23326200

1.71114700

1.40891800

1.63684400

1.99768500

1.75988200

1.54221700

2.49569800

1.54201300

1.35257200

$-3.92496200$

$-4.49300600$

$-4.18990400$

$-3.28074200$

$-3.03537800$

1.16513000

3.02457100

1.11892800

$-0.05553200$

0.21449200

$-0.36593400$

$-1.55213800$

$-1.27369400$

$-1.07412100$

$-1.33149200$

$-2.16516100$

$-2.02474500$

$-2.08660800$

$-0.27181700$

$-1.09335400$

$-2.51269600$

$-3.39711700$

4.14151100

4.84974400

3.44368200

3.94909600

3.46704000
$-0.78222200$

$-0.56374300$

1.64270600

$-0.95267200$

$-0.74761800$

1.57945000

0.57109100

$-1.61030200$

0.77608900

3.01540400

3.70522800

$-1.87120000$

$-1.89727400$

$-1.41971700$

$-2.35633700$

0.06829900

$-0.02613800$

$-0.76329100$

1.26636100

1.37647700

0.32193000

0.35870100

$-0.75277500$

$-0.47732500$

1.77873700

$-1.03283200$

$-0.78901000$

1.50906100

0.45012900

$-1.68573500$

0.67748700

2.86040700

3.59428600

$-1.99688900$

$-2.14601700$

$-1.52986600$

$-1.78099400$

$-0.73238700$

$-1.22327400$

$-1.32638100$

0.71318200

1.19245800
$-1.38708100$

1.45298700

$-0.10296300$

$-1.37006600$

1.49980200

$-0.11260800$

$-1.34969400$

0.13313100

1.31367000

$-0.25947500$

0.37287400

$-2.61333400$

$-2.76522500$

2.88851800

3.02973300

0.20449100

1.13903900

$-0.71984200$

$-0.20541000$

$-1.27509300$

$-0.76995000$

$-0.97590400$

0.76265400

$-1.84772000$

$-0.20599900$

1.41362700

$-1.19239200$

0.48975200

1.75972800

0.20247700

$-0.66290400$

1.02440800

0.39996800

2.73324400

3.21835800

$-2.28789500$

$-1.99251500$

0.14436500

$-0.52609100$

0.73942100

0.06966900

0.93583400 
${ }^{3} \mathrm{~A} 2-5 \mathrm{TS}$

$\begin{array}{lrrr}\text { V } & 1.18031200 & -0.80027500 & -0.02408100 \\ \text { O } & 2.97954600 & -1.27974800 & -0.00024000 \\ \text { O } & 0.42136000 & -0.48215400 & 1.62711800 \\ \text { O } & -0.14962600 & -1.70776700 & -0.87561300 \\ \text { O } & 1.01549400 & 0.88265800 & -0.79179600 \\ \text { Si } & -1.07879200 & 0.19805600 & 1.65883600 \\ \text { Si } & -1.66536500 & -1.03327200 & -0.86225400 \\ \text { Si } & -0.48481200 & 1.57665900 & -0.77501800 \\ \text { O } & -1.00687100 & 1.64818800 & 0.81501700 \\ \text { O } & -2.09541600 & -0.77067900 & 0.73518000 \\ \text { O } & -1.53629900 & 0.50286100 & -1.52137900 \\ \text { O } & -0.45676000 & 3.04521100 & -1.49877400 \\ \text { H } & -1.29366600 & 3.52504200 & -1.53813100 \\ \text { O } & -1.62048500 & 0.38841100 & 3.19142000 \\ \text { H } & -2.52452200 & 0.71120400 & 3.29395100 \\ \text { O } & -2.72975300 & -1.98385700 & -1.66074300 \\ \text { H } & -3.65377500 & -1.70411700 & -1.65278200 \\ \text { C } & 4.08888000 & -0.55383600 & 0.03883100 \\ \text { H } & 4.45711100 & -0.22759500 & -1.05407500 \\ \text { H } & 4.96804500 & -1.07628700 & 0.45805200 \\ \text { C } & 4.11699100 & 0.88828500 & 0.06309800 \\ \text { H } & 3.22210700 & 1.45434200 & -0.22068200 \\ \text { H } & 5.07833000 & 1.39182700 & 0.19253000\end{array}$

${ }^{3} \mathrm{~A} 3$

$\mathrm{V}$

0

0

0

0 


$\begin{array}{lrrr}H & 1.26445300 & 1.54115200 & -3.55752000 \\ \mathrm{C} & -3.85467100 & -0.30220800 & -0.34921200 \\ \mathrm{H} & -4.56602800 & -0.30680200 & -1.18974000 \\ \mathrm{H} & -2.72639800 & 0.87278800 & 0.62861900 \\ \mathrm{C} & -3.26340300 & -1.49004900 & 0.04960800 \\ \mathrm{H} & -2.89630900 & -1.55978300 & 1.08534500 \\ \mathrm{H} & -3.63949600 & -2.41317500 & -0.39991100\end{array}$

${ }^{3} \mathrm{~A} 4$

V

1.12126500

$-0.01080100$

$-0.91312500$

3.20060400

0.00013700

$-0.95561800$

1.19090100

0.09811400

0.98361200

0

0.05251000

$-1.47490800$

$-1.18046900$

0

$-0.03375400$

1.34765300

$-1.34406200$

Si

$-0.26933300$

0.10211600

1.73265400

Si

$-1.40093500$

$-1.47705400$

$-0.38523400$

Si

$-1.48052700$

1.38065600

$-0.52300600$

$-1.17233200$

1.37890200

1.11409900

$-1.10679100$

$-1.27347100$

1.25005400

$-2.22327100$

$-0.08910400$

$-0.84368900$

$-2.42705100$

2.66562100

$-0.89205500$

$-2.48526200$

2.89391500

$-1.82827600$

$-0.11538300$

0.19356100

3.36247100

$-0.93671900$

0.25532200

3.86635800

$-2.24057600$

$-2.84328600$

$-0.71560300$

$-3.12427300$

$-2.90745600$

$-0.33176700$

3.95466700

$-0.73640600$

0.06086000

4.78861900

$-1.30496900$

$-0.36213200$

3.31824900

$-1.23880700$

0.79657100

3.97581000

0.72478100

0.05312000

3.35254900

1.25250400

0.78184000

4.82716500

1.26183900

$-0.37632000$

${ }^{3} \mathrm{~A} 5$

$\mathrm{V}$

0

1.12890400

$-0.83327000$

$-0.18217000$

3.10305400

$-1.28792500$

$-0.18282000$

0.43319200

$-0.75120100$

1.52713600

$-0.25791800$

$-1.59425000$

$-1.12036700$

1.01999900

0.95779700

$-0.70939800$

Si

$-1.04524500$

$-0.03362900$

1.68329500

$-1.73516100$

$-0.87079600$

$-0.97113300$

$\mathrm{Si}$

$-0.46096600$

1.66543100

$-0.54924300$

$-0.93756100$

1.51812500

1.05325200

$-2.11734700$

$-0.82587300$

0.66260500 


$\begin{array}{lrrr}0 & -1.57179000 & 0.74184100 & -1.40475000 \\ 0 & -0.42822000 & 3.22637200 & -1.04864400 \\ H & -1.26422300 & 3.70730500 & -1.00602700 \\ 0 & -1.54701300 & -0.04653800 & 3.24277500 \\ H & -2.44711700 & 0.26200000 & 3.40575300 \\ 0 & -2.86806500 & -1.64784600 & -1.86237000 \\ H & -3.76025000 & -1.27989100 & -1.83801300 \\ C & 4.14981300 & -0.64117800 & -0.07735300 \\ H & 4.98091100 & 1.21459700 & -0.66134700 \\ H & 5.09662500 & -1.21962300 & -0.06888200 \\ C & 4.23282300 & 0.82951000 & 0.05289200 \\ H & 3.25689800 & 1.31396400 & -0.09551100 \\ H & 4.62908900 & 1.05874800 & 1.06047900\end{array}$

${ }^{3} \mathrm{~B} 2$

C

$\mathrm{H}$

$\mathrm{H}$

C

$\mathrm{H}$

$\mathrm{H}$

0

0

0

0

Si

Si

0

0

$\mathrm{H}$

0

$\mathrm{H}$

0

0

0

0

0

0

Si

0

$\mathrm{H}$

0

$\mathrm{H}$

V

4. 54248300

1.20208900

0.28189900

5.33729700

1.91715800

$-0.02356400$

4.66116200

1.05412100

1.37271200

4.67351100

$-0.08320300$

$-0.44901600$

5.40229200

$-0.82401200$

$-0.11145000$

4.17519300

$-0.22026500$

$-1.41176900$

$-2.25146500$

3.36084000

$-0.27752600$

$-1.93162100$

1.10293900

1. 27195100

0.29795800

2. 38705700

$-0.08845000$

$-1.79507600$

0.91639100

$-1.45552200$

$-2.12922300$

$-0.53384000$

1.51932500

$-2.03225400$

$-0.74312800$

$-1.51117200$

$-2.41432600$

$-1.19737300$

0.02729300

$-3.37680600$

$-0.74615400$

2. 54932800

$-3.54577600$

$-1.64132700$

2.86989500

$-3.26837500$

$-1.16254000$

$-2.49144000$

$-3.12651800$

$-1.08049300$

$-3.44331700$

3.30270800

1.87008400

0.05289400

1.41948200

0.21338100

1.42651700

1.49721200

0.08869800

$-1.35758900$

0.88263000

$-2.06899900$

0.12825600

$-0.74255300$

$-1.19341300$

2.11502900

$-0.62286300$

$-1.44448600$

$-1.99231100$

0.93118600

$-1.46154700$

$-1.41536300$

1.69037200

$-2.23410900$

2.65606700

1.56013900

$-2.05135300$

3.59531200

1.90515600

$-2.34127100$

$-2.39125500$

1.67963800

$-3.27022900$

$-2.52787300$

1.67645900

1.26435400

$-0.00581100$ 
V

Si

${ }^{3}$ B2 - 3TS

0

0

0

Si

Si

0

0

$\mathrm{H}$

0

$\mathrm{H}$

0

0

0

0

0

Si

0

$\mathrm{H}$

0

$\mathrm{H}$

Si

V

0

C

$\mathrm{H}$

$\mathrm{H}$

C

$\mathrm{H}$

$\mathrm{H}$

0

v

${ }^{3}$ B2-4TS

$\mathrm{V}$

0

C

$\mathrm{H}$

$\mathrm{H}$

C

$\mathrm{H}$
$-1.43868900$

2.03685800

$-0.14334400$

0.82689400

$-1.32813900$

1.60202600

1.21842700

1.55970700

$-1.26063900$

$-0.92867500$

1.99958600

0.63649500

1.74039500

1.16582400

1.39951700

1.81541500

0.07965700

$-1.77228100$

2.37400300

$-0.37102700$

1.18344500

2.57506300

$-0.55713800$

$-0.44459500$

2.81647600

0.22850100

$-3.05325200$

3.59700300

0.78663500

$-2.94399900$

3.83726000

$-0.53629900$

1.89170900

3.85335200

$-0.63147200$

2.85262200

$-1.80080500$

$-0.43900300$

$-1.11491800$

$-1.26582000$

$-0.61380300$

1.52973200

$-0.27224800$

$-2.42014100$

$-0.17617600$

0.58180400

$-0.89177200$

$-2.23723100$

1.29701900

$-1.46470500$

1.76966100

$-0.26194100$

$-1.90635900$

1.40596600

$-1.37400100$

$-2.71267300$

$-2.59467800$

$-1.31534500$

$-2.49352100$

$-3.53340300$

$-0.75796000$

$-3.08793800$

2.42625300

$-0.22449800$

$-3.89238400$

2.45412100

$-0.72995400$

$-1.63186000$

$-1.54996800$

$-1.96651100$

0.57921300

0.34438300

$-3.40919800$

1.75335100

$-0.87972400$

$-4.42354900$

1.10486600

$-0.09449300$

$-5.19588000$

0.58174900

$-0.67467600$

$-4.32067800$

2. 36025800

$-0.28286400$

$-3.90313400$

0.61120100

1.15878800

$-3.59661900$

1.37843400

1.89413000

$-4.37478600$

$-0.25876300$

1.62856500

1.20371700

3.70306900

0.47775000

0.79610200

2. 20315200

0.33041000

$-1.74777900$

0.92469300

$-0.62569500$

$-3.53924200$

1.37901800

$-0.76314700$

$-4.70240600$

1.23472100

0.79325000

$-5.23729000$

2.18466700

0.85802900

$-4.04661600$

0.91802000

1.60778200

$-4.64818600$

0.51267600

$-0.47838400$

$-4.36970400$

$-0.55089200$

$-0.42905300$ 


$\begin{array}{lrrr}H & -5.47135400 & 0.68256400 & -1.18639600 \\ O & -1.79605900 & 0.17938100 & 1.06285900 \\ \text { O } & -0.44122800 & 2.17807200 & -0.74288100 \\ \text { O } & -1.29623600 & -0.48971500 & -1.66301900 \\ \text { Si } & -1.01465600 & -1.15126200 & 1.61808100 \\ \text { Si } & -0.49022800 & -1.88392100 & -1.28402900 \\ \text { O } & -0.67000600 & -2.15207900 & 0.33684400 \\ \text { O } & -1.96911800 & -1.90049600 & 2.72421100 \\ \text { H } & -1.58753800 & -2.66324600 & 3.17708600 \\ \text { O } & -1.06497900 & -3.19190400 & -2.08860300 \\ H & -0.64501800 & -3.38468600 & -2.93640600 \\ \text { O } & 1.92781900 & 3.50804800 & -0.58957300 \\ \text { O } & 1.53105400 & 1.47264000 & 1.24038800 \\ \text { O } & 2.05761800 & 0.85776700 & -1.38459700 \\ \text { O } & 2.48624400 & -0.90970400 & 0.54138900 \\ O & 0.43516800 & -0.73881600 & 2.31451700 \\ O & 1.11208200 & -1.67790700 & -1.64793000 \\ \text { Si } & 2.35836100 & -0.76148100 & -1.10578000 \\ O & 2.95745100 & -0.06561900 & 3.01300400 \\ \text { H } & 2.66082700 & 0.24476000 & 3.87789200 \\ \text { O } & 3.70114000 & -1.28234300 & -1.87904400 \\ \text { H } & 4.52337500 & -0.81093100 & -1.69401400 \\ \text { V } & 1.26896700 & 2.10336400 & -0.39533700 \\ \text { Si } & 1.84908500 & -0.06624400 & 1.80917500\end{array}$

$\begin{array}{lrrr}{ }^{3} \text { B2-5TS } & & & \\ \text { V } & -1.78940700 & 0.94351800 & -0.49667100 \\ \text { O } & -3.51779500 & 1.54365700 & -0.77259000 \\ \text { C } & -4.69228200 & 1.25625600 & -0.21754300 \\ \text { H } & -5.56175300 & 1.45834500 & -0.87048100 \\ \text { H } & -4.91399600 & 1.93781000 & 0.72977800 \\ \text { C } & -4.88448700 & 0.27808200 & 0.82287700 \\ \text { H } & -4.02571500 & -0.08536200 & 1.39824100 \\ \text { H } & -5.90543900 & 0.04858400 & 1.13967700 \\ \text { O } & 1.85938500 & 3.57904000 & -0.28173300 \\ \text { O } & 1.96647000 & 1.00790200 & -1.32363600 \\ \text { O } & -0.48765700 & 2.21990200 & -0.40984900 \\ \text { O } & 1.61233000 & 1.37402600 & 1.37946900 \\ \text { Si } & 2.35078400 & -0.61023900 & -1.18958200 \\ \text { Si } & 1.89963600 & -0.21646500 & 1.78916500 \\ 0 & 2.51597100 & -0.92798600 & 0.42327000 \\ \text { O } & 3.77633400 & -0.97031600 & -1.91042300 \\ \text { H } & 3.79901600 & -0.91965600 & -2.87446400 \\ \text { O } & 2.91590100 & -0.35937500 & 3.06335400\end{array}$




$\begin{array}{lrrr}H & 3.78489500 & 0.05192600 & 2.97284400 \\ 0 & -1.33247000 & -0.35236600 & -1.69780300 \\ 0 & -1.77902300 & -0.00368400 & 1.07184100 \\ 0 & -0.57892000 & -2.19137400 & 0.10328200 \\ 0 & 1.12583700 & -1.50053500 & -1.83478600 \\ 0 & 0.49855200 & -0.95318400 & 2.22242900 \\ \text { Si } & -0.93037100 & -1.35291500 & 1.48897400 \\ 0 & -1.07414600 & -2.90691100 & -2.48553600 \\ \text { H } & -0.49929400 & -3.66712800 & -2.63902800 \\ 0 & -1.84698200 & -2.23868400 & 2.52467200 \\ \text { H } & -1.37120000 & -2.84079600 & 3.11061400 \\ \text { V } & 1.23270300 & 2.14891300 & -0.17657600 \\ \text { Si } & -0.48233500 & -1.74013700 & -1.49499900\end{array}$

${ }^{3} \mathrm{~B} 3$

0

0

$-1.32774000$

1.66070000

1.18175500

0.74600900

1.99824500

$-0.79404200$

$-1.87474800$

0.99198100

$-1.43076600$

Si

$-1.78818100$

0.19117200

1.82413100

Si

$-2.40959000$

$-0.54490700$

$-1.05981100$

$-2.53369200$

$-0.62131100$

0.58481300

$-2.76758300$

0.36223200

3.12316700

$-3.57097000$

0.88286700

2.99666400

$-3.89192800$

$-0.85189700$

$-1.68301300$

$-3.94837200$

$-0.94951400$

$-2.64203100$

1.87362300

$-0.15559100$

1.04308200

1.29120900

$-0.82951000$

$-1.70014300$

0.37193700

$-2.26674600$

0.37432900

$-0.47416900$

$-0.64787700$

2. 33158700

$-1.30420400$

$-1.63432800$

$-1.60924300$

Si

0.26700900

$-2.04395200$

$-1.26776000$

1.68243200

$-2.18755000$

2.77377900

1.13614900

$-2.64768000$

3.42360100

0.67270400

$-3.40401500$

$-2.09033600$

0.04855100

$-4.13893100$

$-2.04071900$

0.87593500

$-1.31574300$

1.63967700

1.87324800

0.55857100

$-0.67375300$

4.13503400

1.61032400

0.98066500

4.25229500

0.74506800

$-0.02660000$

4.50388500

$-0.29321400$

0.22885500

3.88501800

1.12909600

1.78867500

4.05480600

1.12179300

$-1.32349100$

3.91356200

2.17937100

$-1.57568700$

4.29824100

0.41942200

$-2.12510400$ 
0

V

${ }^{3} \mathrm{~B} 4$

V

0

C

$\mathrm{H}$

$\mathrm{H}$

C

$\mathrm{H}$

$\mathrm{H}$

0

0

0

Si

Si

0

0

$\mathrm{H}$

0

$\mathrm{H}$

0

0

0

0

0

0

Si

0

$\mathrm{H}$

0

$\mathrm{H}$

V

Si

${ }^{3} \mathrm{~B} 5$

V

0

C

$\mathrm{H}$

$\mathrm{H}$

C

$\mathrm{H}$
$-1.41423100$

3.64371500

$-0.75558400$

$-0.95230700$

2.17440500

$-0.47848200$

$-1.73532900$

0.87046800

$-0.63623200$

$-3.72549800$

1.33636900

$-0.70879400$

$-4.50999700$

1.58099600

0.50397800

$-5.18728400$

2.43422000

0.40088600

$-3.92249100$

1.50692600

1.42480300

$-4.73253600$

0.36639800

$-0.27963500$

$-4.31204000$

$-0.57703800$

0.08354600

$-5.57674700$

0.29998200

$-0.97261800$

$-1.85359900$

0.13458200

1.07090900

$-0.43300900$

2.16336200

$-0.76116700$

$-1.34104900$

$-0.57243600$

$-1.68003300$

$-1.01220200$

$-1.15265600$

1.63609600

$-0.47885800$

$-1.91914200$

$-1.27041700$

$-0.63620500$

$-2.15082100$

0.36012300

$-1.92527200$

$-1.93727100$

2.75486700

$-1.50242500$

$-2.68007600$

3.20414900

$-1.00337500$

$-3.26974400$

$-2.04190000$

$-0.51570100$

$-3.50922200$

$-2.83999300$

1.90734100

3.53263700

$-0.63356800$

1.53335500

1.50833100

1.22655600

2.06645900

0.86444300

$-1.39747000$

2.47390300

$-0.88855700$

0.54779700

0.42695200

$-0.68729200$

2. 32254000

1.11692900

$-1.67413700$

$-1.64562600$

2.35781700

$-0.75122800$

$-1.10173300$

2.95293600

$-0.02214100$

3.00941700

2.65754100

0.30346200

3.86900300

3.70731000

$-1.28229100$

$-1.85935600$

4.52324300

$-0.79708300$

$-1.68255700$

1.26693000

2.11982900

$-0.42120800$

1.84230500

$-0.02573500$

1.80558600

$-1.76326300$

0.94753300

$-0.41578400$

$-3.64077800$

1.58565600

$-0.72681000$

$-4.75657900$

1.33543900

$-0.26039400$

$-5.61495900$

1.87827000

$-0.70627300$

$-5.65362600$

0.86429000

1.59336600

$-5.03257200$

0.37185400

0.82481400

$-4.11150500$

$-0.04023400$

1.26044800 
H

0

0

0

0

Si

Si

0

0

$\mathrm{H}$

0

$\mathrm{H}$

0

0

0

0

0

Si

0

$\mathrm{H}$

0

$\mathrm{H}$

V

Si

${ }^{3} \mathrm{C} 2$

C

$\mathrm{H}$

$\mathrm{H}$

C

$\mathrm{H}$

$\mathrm{H}$

0

0

0

Si

Si

0

0

$\mathrm{H}$

0

$\mathrm{H}$

0

0
$-5.65825700$

1.88731900

1.97450000

$-0.45808400$

1.65334500

2.33936000

1.93188100

2.52017200

3.75226100

3.76072800

2. 97188000

3.83857500

$-1.37849800$

$-1.77923300$

$-0.56685800$

1.09728100

0.53546700

$-0.90756800$

$-1.10167900$

$-0.55170300$

$-1.77420800$

$-1.25986600$

1.25864800

$-0.50305900$

$-4.49782100$

$-5.27421300$

$-4.44575600$

$-4.84881300$

$-4.06667400$

$-5.87221600$

1.86852500

$-0.25892100$

1.69730100

2.22389600

2.03737700

2. 52661400

3.50663800

3.70455900

3.21604400

2. 93235100

$-3.25634000$

$-1.34429600$
$-0.43910800$

3.59474500

1.06199100

2. 24121200

1.32566800

$-0.56404800$

$-0.28137200$

$-0.94617300$

$-0.91394000$

$-0.83319200$

$-0.47654700$

$-0.06070400$

$-0.30027500$

$-0.05581300$

$-2.17617900$

$-1.41469900$

$-1.02499900$

$-1.40651800$

$-2.82498200$

$-3.60823800$

$-2.36707700$

$-2.95486800$

2.16185700

$-1.67910800$

1.13717300

1.90584500

0.53245400

0.29200800

$-0.06485700$

$-0.07549900$

1.21879500

2. 33078500

1.12149400

$-0.38133900$

$-0.49530100$

$-0.99857700$

$-0.50256000$

$-1.37754300$

$-0.77399200$

$-1.09035800$

1.81920200

0.11253400
0.40615500

$-0.16791600$

$-1.30855500$

$-0.32214400$

1.41303000

$-1.23893700$

1.75966400

0.35786400

$-1.99060100$

$-2.95276300$

3.00852000

2. 91716100

$-1.70290000$

1.12922200

0.03989100

$-1.90444000$

2.19180400

1.47084400

$-2.55829300$

$-2.68482200$

2.48313300

3.05069600

$-0.10813900$

$-1.54547700$

$-0.37425200$

$-0.55569200$

$-1.30830600$

0.79455700

1.46952900

0.90398800

$-1.21483300$

$-0.01155200$

1.43282500

$-1.41925700$

1.58712400

0.08994300

$-2.42350800$

$-2.78046700$

2.68208400

3.54906200

$-0.21923400$

$-1.50836200$ 
0

0

0

0

Si

0

$\mathrm{H}$

0

$\mathrm{H}$

V

Si

Si

0

$\mathrm{H}$

${ }^{3} \mathrm{C} 2-3 \mathrm{TS}$

0

0

0

Si

Si

0

0

$\mathrm{H}$

0

$\mathrm{H}$

0

0

0

0

0

Si

0

$\mathrm{H}$

0

$\mathrm{H}$

Si

v

0

C

$\mathrm{H}$

$\mathrm{H}$

C

$\mathrm{H}$
$-1.50594400$

$-0.76552100$

0.92594500

0.66573300

$-0.84842600$

$-1.42037700$

$-1.24308600$

$-1.70291200$

$-2.41721200$

$-1.62936000$

$-0.65787100$

1.38142400

2.16662000

1.77486700

$-1.14497800$

0.82714400

$-1.66345800$

$-1.81480300$

$-2.36791700$

$-2.62443900$

$-2.79211900$

$-3.55374700$

$-3.80437400$

$-3.78315700$

1.75684300

1.22139300

0.23681500

$-0.63938300$

$-1.33910000$

0.21428300

1.30933700

1.22658400

0.69192600

0.21692300

0.68000800

1.90711400

3.37950200

4.37313300

5.12782800

4. 30101900

3.83509800

3.54910600
0.05644300

$-2.11096600$

$-1.19124500$

$-1.29863400$

$-1.46081000$

$-2.37247000$

$-2.21057000$

$-2.43241900$

$-2.94246100$

1.21491200

$-1.39560100$

2.07215700

3.49583600

4.26362900

1.25662300

$-0.12612700$

$-2.04344000$

2.02961900

1.39928500

$-2.68424800$

$-3.61950700$

2.40217300

2.00005100

$-0.11493100$

$-1.61325600$

0.12152200

0.28293600

$-0.15211300$

1.69005600

1.10643800

1.97463400

1.17568000

$-0.70124300$

0.30025400

$-1.44530300$

$-0.30454800$

1.72247100

$-0.40036300$

$-1.17591700$

0.59626800

0.45463500

1.16809900

2.99915600

2.84050500

$-0.44582900$

$-1.94436100$

$-0.83210300$

$-2.82882300$

$-0.32918400$

1.13597100

$-0.65799800$

$-1.50571700$

$-2.37321200$

0.31009600

$-0.71053800$

2.25906100

$-1.49101200$

$-1.67070800$

$-1.93829600$

$-1.29462600$

$-2.50567300$

2.75245700

$-2.22824200$

3.67365200

$-3.17977000$

$-2.25289100$

$-4.01402600$

$-2.14929200$

$-1.48964500$

1.63317100

0.60584300

$-0.39017100$

1.82784300

0.75758000

1.11468400

$-0.00413800$

0.59056100

0.59779400

2.37796000

0.12926700

0.58228700

$-1.23435700$

1.32210600

$-2.00438900$ 
$\mathrm{H}$

Si

0

$\mathrm{H}$

${ }^{3} \mathrm{C} 2-4 \mathrm{TS}$

$\mathrm{V}$

0

C

$\mathrm{H}$

$\mathrm{H}$

C

$\mathrm{H}$

$\mathrm{H}$

0

0

0

Si

Si

0

0

$\mathrm{H}$

0

$\mathrm{H}$

0

0

0

0

0

Si

0

$\mathrm{H}$

0

$\mathrm{H}$

Si

0

$\mathrm{H}$

Si

${ }^{3} \mathrm{C} 2-5 \mathrm{TS}$

$\mathrm{V}$

0

C

$\mathrm{H}$
4.28138900

$-0.80391600$

$-1.24113200$

$-0.57794000$

$-1.68672500$

$-3.48474400$

$-4.63489100$

$-5.17729500$

$-3.98098400$

$-4.58550100$

$-4.29556600$

$-5.40799100$

$-1.75963200$

$-0.39445600$

$-1.23297700$

$-0.94321500$

$-0.37622000$

$-0.56953000$

$-1.87312800$

$-1.47703700$

$-0.87729000$

$-0.40675800$

1.41728100

1.97105300

2.57048100

0.49362100

1.22370500

2. 39985200

2. 91717000

2. 53940300

3.76351300

4.55084100

1.84875900

1.89209800

1. 39105500

1.20825500
$-0.32232700$

2.16797800

3.71982100

4.39558700

0.84829700

1.30546000

1. 35123800

2. 29704800

1.14090900

0.46556600

$-0.57928300$

0.53696300

0.25114600

2.08787300

$-0.66282100$

$-0.99648800$

$-1.97793900$

$-2.10779800$

$-1.66769700$

$-2.39708300$

$-3.38123100$

$-3.64284300$

1.67011000

0.86938800

$-0.68200700$

$-0.47427700$

$-1.74037200$

$-0.67975900$

0.50289500

0.64545500

$-1.16264200$

$-0.62170400$

0.25670000

3.53063200

4.11644000

2.06771100
$-1.66164300$

$-0.44642300$

$-0.72931100$

$-0.54052300$

$-0.72846500$

$-0.90357900$

0.63740100

0.58037300

1.48681900

$-0.52490400$

$-0.33789200$

$-1.25036300$

1.02228100

$-0.96938800$

$-1.63113800$

1.70268300

$-1.11413900$

0.52411600

2.87953200

3.37295200

$-1.80247700$

$-2.60389800$

1.01351100

$-1.44670700$

0.61097900

2. 35320700

$-1.47270900$

$-1.04102600$

2.98112300

3.85781900

$-1.80570200$

$-1.66542900$

1.76504200

$-0.87038300$

$-1.45129400$

$-0.58189500$

$-1.66900100$

1.07668200

$-0.43847300$

$-3.38853700$

1.72708600

$-0.68207800$

$-4.58547800$

1.33362400

$-0.26848200$

$-5.41863900$

1.63205400

$-0.93085400$ 


\begin{tabular}{|c|c|c|c|}
\hline $\mathrm{H}$ & -4.88588300 & 1.82145700 & 0.78181000 \\
\hline $\mathrm{C}$ & -4.82496000 & 0.21139400 & 0.60564500 \\
\hline $\mathrm{H}$ & -4.00191900 & -0.20432500 & 1.19606100 \\
\hline $\mathrm{H}$ & -5.85785300 & -0.09252300 & 0.79387300 \\
\hline 0 & 1.95482100 & 1.11628300 & -1.27298700 \\
\hline 0 & -0.32066500 & 2.27829000 & -0.36291800 \\
\hline 0 & 1.58794900 & 1.38251100 & 1.33424200 \\
\hline Si & 2.33630400 & -0.48890900 & -1.23669500 \\
\hline Si & 1.92761900 & -0.18922600 & 1.72942100 \\
\hline 0 & 2.53922000 & -0.88929800 & 0.36254900 \\
\hline 0 & 3.70585600 & -0.71429700 & -2.10628900 \\
\hline $\mathrm{H}$ & 3.85231700 & -1.60585800 & -2.44607500 \\
\hline 0 & 3.03852900 & -0.28980800 & 2.92939300 \\
\hline $\mathrm{H}$ & 2.68697000 & -0.41828100 & 3.81906400 \\
\hline 0 & -1.27717800 & -0.23237400 & -1.65802400 \\
\hline 0 & -1.71212900 & 0.08573900 & 1.11627300 \\
\hline 0 & -0.60032400 & -2.15120500 & 0.10001100 \\
\hline 0 & 1.12117200 & -1.41761800 & -1.85092300 \\
\hline 0 & 0.54456300 & -0.95479400 & 2.20270100 \\
\hline Si & -0.91419600 & -1.29902100 & 1.49063700 \\
\hline 0 & -1.07919200 & -2.87456700 & -2.40870300 \\
\hline $\mathrm{H}$ & -0.94721300 & -2.77703000 & -3.36006300 \\
\hline 0 & -1.85455900 & -2.17411400 & 2.51767400 \\
\hline $\mathrm{H}$ & -1.53111200 & -3.05359200 & 2.75034700 \\
\hline Si & -0.48030300 & -1.65922700 & -1.47804000 \\
\hline Si & 1.29320400 & 2.12726000 & -0.12592100 \\
\hline 0 & 1.95987100 & 3.62360500 & -0.18892100 \\
\hline $\mathrm{H}$ & 2.92100300 & 3.66850800 & -0.10988900 \\
\hline \multicolumn{4}{|l|}{${ }^{3} \mathrm{C} 3$} \\
\hline 0 & -1.15510100 & 1.85725900 & 0.93769700 \\
\hline 0 & 0.73818100 & 1.89644400 & -1.02258600 \\
\hline 0 & -1.75785000 & 0.98967300 & -1.48727000 \\
\hline $\mathrm{Si}$ & -1.74277900 & 0.54161000 & 1.75723800 \\
\hline $\mathrm{Si}$ & -2.43994500 & -0.43938900 & -1.00352500 \\
\hline 0 & -2.59161700 & -0.34789800 & 0.64143200 \\
\hline 0 & -2.66895500 & 0.97209200 & 3.03626000 \\
\hline $\mathrm{H}$ & -3.42773300 & 1.53796400 & 2.84583500 \\
\hline 0 & -3.92341900 & -0.66917800 & -1.65897000 \\
\hline $\mathrm{H}$ & -3.95106500 & -1.19317100 & -2.46910600 \\
\hline 0 & 1.84813300 & -0.10979100 & 1.01443300 \\
\hline 0 & 1.16670600 & -1.00528600 & -1.64774800 \\
\hline 0 & 0.24230300 & -2.20365800 & 0.57488500 \\
\hline 0 & -0.50729800 & -0.35526000 & 2.35986600 \\
\hline
\end{tabular}


0

Si

0

$\mathrm{H}$

0

$\mathrm{H}$

Si

$\mathrm{V}$

0

C

$\mathrm{H}$

$\mathrm{H}$

C

$\mathrm{H}$

$\mathrm{H}$

Si

0

$\mathrm{H}$

${ }^{3} \mathrm{C} 4$

$\mathrm{V}$

0

C

$\mathrm{H}$

$\mathrm{H}$

C

$\mathrm{H}$

$\mathrm{H}$

0

0

0

Si

Si

0

0

$\mathrm{H}$

0

$\mathrm{H}$

0

0

0

0

0

Si
$-1.45216700$

0.11125400

1.57509500

1.00795000

0.44240900

$-0.17655400$

0.80467500

1.82124400

4.06905700

4.19134000

4.44617800

3.75441500

4.00627000

3.85129700

4.26218900

$-0.85203000$

$-1.32778000$

$-0.75899100$

$-1.68496300$

$-3.67583800$

$-4.68480900$

$-5.55019500$

$-4.26797600$

$-4.42069100$

$-3.80811800$

$-5.08818300$

$-1.25926100$

$-0.42527100$

$-1.82480200$

$-0.36442700$

$-0.91477700$

$-0.49768700$

$-0.88191200$

$-0.37402500$

$-1.75951200$

$-1.22501400$

1.91360200

1.45092000

2.56435000

1. 24512700

0.49482400

1.84359900
$-1.69077100$

$-2.13104100$

$-1.94687400$

$-2.29983600$

$-3.58205300$

$-4.29773700$

$-1.15269200$

0.45566900

1.63608300

0.72121100

$-0.30192100$

1.19603100

1.03094400

2.07224500

0.29272500

2.12251300

3.64754000

4.11979400

$-0.75772900$

$-0.76156600$

$-1.26293400$

$-0.83119100$

$-0.35775900$

$-0.28509300$

$-0.24072000$

$-0.94468300$

0.55340200

0.15631600

$-1.63719200$

0.37219200

$-1.63930100$

1.27934400

$-2.48827900$

0.20609400

0.79059100

$-1.64803600$

$-2.05525400$

$-1.03220100$

$-0.19574600$

1.01615400

2.03779000

$-1.06431500$

0.95612000

1.74207500

2.08322800

0.59113700

3.44256800

$-1.74240100$

4.23277400

$-1.51984500$

1.64718100

2.97225500

2.02529000

3.68176000

$-0.80055600$

$-1.49496300$

$-1.76631400$

0.92563700

0.62124900

0.64216400

1.81375700

$-1.41416700$

0.32922600

2. 35431100

$-0.38322100$ 


\begin{tabular}{|c|c|c|c|}
\hline 0 & 3.83090600 & 1.13958200 & -1.65828800 \\
\hline $\mathrm{H}$ & 3.78246600 & 1.66993500 & -2.46323400 \\
\hline 0 & 2.81985200 & -0.68529100 & 3.03059700 \\
\hline $\mathrm{H}$ & 3.65100800 & -1.13683700 & 2.83718700 \\
\hline Si & 2.39370000 & 0.70501000 & -1.00160600 \\
\hline Si & 1.17978300 & -2.05788500 & -0.68689900 \\
\hline 0 & 1.87054300 & -3.49834300 & -1.06845900 \\
\hline $\mathrm{H}$ & 1.41014100 & -4.01173800 & -1.74360300 \\
\hline \multicolumn{4}{|l|}{ C5 } \\
\hline V & -1.62209800 & 1.13587200 & -0.25233300 \\
\hline 0 & -3.48416500 & 1.85395600 & -0.47757100 \\
\hline C & -4.62047300 & 1.45918400 & -0.20024000 \\
\hline $\mathrm{H}$ & -5.46546500 & 2.09383300 & -0.53774500 \\
\hline $\mathrm{H}$ & -5.72869400 & 0.39148100 & 1.26139200 \\
\hline C & -4.93334900 & 0.20614900 & 0.52084400 \\
\hline $\mathrm{H}$ & -4.04019400 & -0.22849000 & 0.99139000 \\
\hline $\mathrm{H}$ & -5.35505900 & -0.50647900 & -0.21431900 \\
\hline 0 & 1.96895200 & 1.19996900 & -1.22831100 \\
\hline 0 & -0.24647500 & 2.32547700 & -0.14596400 \\
\hline 0 & 1.69334700 & 1.24768700 & 1.40287200 \\
\hline Si & 2.28450300 & -0.41538500 & -1.34190800 \\
\hline Si & 1.98881200 & -0.36177400 & 1.65358200 \\
\hline 0 & 2.51844000 & -0.96457100 & 0.20781400 \\
\hline 0 & 3.61748800 & -0.61451600 & -2.27386700 \\
\hline $\mathrm{H}$ & 3.72794200 & -1.48482500 & -2.67645300 \\
\hline 0 & 3.14441600 & -0.59735100 & 2.79203000 \\
\hline $\mathrm{H}$ & 2.82262600 & -0.80257900 & 3.67852700 \\
\hline 0 & -1.38370800 & -0.07342800 & -1.62275600 \\
\hline 0 & -1.69986800 & 0.02129200 & 1.22961200 \\
\hline 0 & -0.63275600 & -2.10418400 & -0.03223200 \\
\hline 0 & 1.01494700 & -1.23947300 & -1.99383600 \\
\hline 0 & 0.60172100 & -1.11947800 & 2.12386600 \\
\hline Si & -0.89195800 & -1.38743400 & 1.44623400 \\
\hline 0 & -1.20613900 & -2.65005900 & -2.56792000 \\
\hline $\mathrm{H}$ & -1.04619600 & -2.51657500 & -3.51048600 \\
\hline 0 & -1.77815100 & -2.36735500 & 2.42590900 \\
\hline $\mathrm{H}$ & -1.39377600 & -3.23129500 & 2.62078400 \\
\hline Si & -0.57250300 & -1.50203900 & -1.57393400 \\
\hline Si & 1.36604500 & 2.12345500 & 0.02309300 \\
\hline 0 & 2.07483200 & 3.60206800 & 0.06577300 \\
\hline $\mathrm{H}$ & 3.03836600 & 3.61208900 & 0.12467700 \\
\hline
\end{tabular}




\begin{tabular}{|c|c|c|c|}
\hline v & 0.59336700 & 0.01595000 & 0.55353300 \\
\hline 0 & 2.13012900 & 0.03041000 & 1.01430100 \\
\hline 0 & 0.06311000 & 1.36641900 & -0.43081800 \\
\hline 0 & -0.59852700 & 0.00932900 & 1.84433400 \\
\hline 0 & 0.09472900 & -1.36444300 & -0.40712600 \\
\hline Si & -1.55385200 & 1.42722300 & -0.95311400 \\
\hline Si & -2.23971400 & -0.00880100 & 1.43296200 \\
\hline Si & -1.51205400 & -1.43990000 & -0.95621700 \\
\hline 0 & -1.76221400 & -0.01263900 & -1.76824600 \\
\hline 0 & -2.45579700 & 1.31311700 & 0.43770100 \\
\hline 0 & -2.41450400 & -1.34115800 & 0.44352000 \\
\hline 0 & -1.82756700 & -2.71366400 & -1.90098100 \\
\hline $\mathrm{H}$ & -1.50136600 & -3.58878800 & -1.65124400 \\
\hline 0 & -1.93320000 & 2.75982900 & -1.78594100 \\
\hline $\mathrm{H}$ & -1.40925900 & 3.00496600 & -2.56089300 \\
\hline 0 & -3.14189300 & -0.01614500 & 2.77479200 \\
\hline $\mathrm{H}$ & -4.10039100 & 0.09256600 & 2.71260300 \\
\hline C & 4.73858600 & 1.65455000 & 0.66937500 \\
\hline $\mathrm{H}$ & 4.94951600 & 1.60284700 & 1.75304800 \\
\hline $\mathrm{H}$ & 5.70778800 & 1.83860600 & 0.16752400 \\
\hline $\mathrm{H}$ & 4.11885200 & 2.55402500 & 0.49892500 \\
\hline C & 4.59093000 & -1.75656800 & 0.53470500 \\
\hline $\mathrm{H}$ & 3.89264300 & -2.58048400 & 0.29782800 \\
\hline $\mathrm{H}$ & 5.54041900 & -1.98805200 & 0.01521600 \\
\hline $\mathrm{H}$ & 4.80318800 & -1.80900000 & 1.61813700 \\
\hline C & 3.05327800 & 0.11134200 & -1.83597000 \\
\hline $\mathrm{H}$ & 2.37999300 & -0.73923800 & -2.05024000 \\
\hline $\mathrm{H}$ & 2.46981800 & 1.03807300 & -1.98685100 \\
\hline $\mathrm{H}$ & 3.83424800 & 0.09806900 & -2.61968100 \\
\hline $\mathrm{Al}$ & 3.87806900 & 0.00814400 & -0.01826200 \\
\hline
\end{tabular}

${ }^{1} \mathrm{A6}$-7TS

$\begin{array}{lrrr}\text { V } & 0.57002400 & 0.00573300 & -0.36865800 \\ 0 & 2.33811900 & 0.15832700 & -0.00634300 \\ 0 & 0.09143400 & 0.17407700 & 1.42818500 \\ 0 & -0.25260100 & -1.51419300 & -0.82617400 \\ 0 & -0.32111500 & 1.34424100 & -1.15507000 \\ \text { Si } & -1.53802100 & 0.17748100 & 1.68421800 \\ \text { Si } & -1.90718600 & -1.53082400 & -0.58849700 \\ \text { Si } & -1.98267700 & 1.31734500 & -0.90978800 \\ 0 & -2.20757400 & 1.39764400 & 0.73945600 \\ 0 & -2.16559000 & -1.24052600 & 1.03239900 \\ 0 & -2.52381100 & -0.18874900 & -1.38043000 \\ 0 & -2.78798500 & 2.46422400 & -1.74731100\end{array}$


$\mathrm{H}$

0

$\mathrm{H}$

0

$\mathrm{H}$

C

$\mathrm{H}$

$\mathrm{H}$

$\mathrm{H}$

C

$\mathrm{H}$

$\mathrm{H}$

$\mathrm{H}$

C

$\mathrm{H}$

$\mathrm{H}$

$\mathrm{H}$

Al

${ }^{1 B} 6-7 T S$

0

0

0

0

Si

Si

0

0

$\mathrm{H}$

0

$\mathrm{H}$

0

0

0

0

0

Si

0

$\mathrm{H}$

0

H

V

V

Si

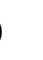

$-2.42348800$

$-1.89911600$

$-2.83457900$

$-2.54473000$

$-3.50760900$

3.03281800

3.81532200

3.32467400

2.07494000

4.70287900

4.78602400

5.70496300

4.01436100

5.10201600

5.98150400

5.49117400

4.49627500

4.08567400
3.35830500

0.34946600

0.35877500

$-2.93182900$

$-2.99288100$

0.57224200

$-0.12565400$

1.62662600

0.38570900

$-1.93879700$

$-2.36617700$

$-2.02391400$

$-2.58650800$

1.45096300

1.64225100

1.28678200

2. 37281700

$-0.09014800$
$-1.72825700$

3. 26948800

3.50885200

$-1.12812900$

$-1.17399100$

1.73377200

2.08036600

1.69620200

2.22703400

$-0.17147700$

$-1.18775500$

0.28591400

0.39758100

$-0.87465100$

$-0.23298400$

$-1.89581900$

$-0.89366900$

$-0.24167800$

2.13241600

$-3.64805100$

0.60194200

2.20516800

$-1.04785000$

1.55661000

$-0.17696000$

$-2.19564400$

0.37960000

2.18286800

$-1.50067800$

$-1.15340400$

2.65928200

0.55260900

1.44169600

2.59994500

0.06540700

$-1.56656900$

3.07517900

0.78064500

$-0.14672800$

3.87868100

0.92068300

2.46692000

4.63358600

0.31919300

2.48623900

3.77918500

0.10224500

$-2.69885300$

4.55495400

$-0.45122000$

$-2.54467600$

$-1.01391300$

0.51523500

1. 36377700

$-1.16705300$

0.09305000

$-1.33686300$

$-0.01901600$

2. 28186200

$-0.35589000$

1.40873800

1.54479000

1.83163500

1.31328300

0.85959800

$-2.19131800$

$-0.19978100$

1.35399200

$-1.73034600$

$-0.74092000$

3.14808700

2.08077400

$-0.39116400$

3.30093600

2.96779300

$-0.87979500$

2. 21547200

$-2.94692800$

$-0.28381800$

2.77120200

$-3.46498700$

$-1.31558800$

$-0.82166600$

0.25743800

1.57938200

$-2.20699100$

0.35700500

$-0.09166300$

1.90371600

1.24231800 


$\begin{array}{lrrr}\text { O } & -3.03769300 & -1.26093200 & 0.03176800 \\ \mathrm{C} & -4.24926700 & 1.60410800 & 0.35264500 \\ \mathrm{H} & -5.17788900 & 2.19473000 & 0.27430800 \\ \mathrm{H} & -3.75272400 & 1.89996700 & 1.29449200 \\ \mathrm{H} & -3.57607900 & 1.91305100 & -0.46548200 \\ \mathrm{C} & -3.84211400 & -1.28340000 & -1.74558000 \\ \mathrm{H} & -2.98828100 & -0.88143000 & -2.29304400 \\ \mathrm{H} & -4.01858100 & -2.35863000 & -1.81021300 \\ \mathrm{H} & -4.75870100 & -0.68160800 & -1.90173500 \\ \mathrm{C} & -6.09317400 & -1.35532600 & 0.87832900 \\ \mathrm{H} & -6.26069500 & -1.25838200 & 1.96651400 \\ \mathrm{H} & -7.01665500 & -1.00070400 & 0.38632100 \\ \mathrm{H} & -5.98276500 & -2.43020800 & 0.65675600 \\ \text { Al } & -4.53934200 & -0.32294800 & 0.31688100\end{array}$

${ }^{1}$ B7

0

0

0

0

Si

Si

0

0

$\mathrm{H}$

0

$\mathrm{H}$

0

0

0

0

0

Si

0

$\mathrm{H}$

0

$\mathrm{H}$

V

V

Si

0

C

$\mathrm{H}$

$\mathrm{H}$
2.15974900
1.58598200
-0.07955500
2.56947600
1.81202600
2.92025100
2.73888400
2.49862300
3.26136100
4.41646000
5.14300600

$-1.73581300$

$-0.61508800$

$-0.19409200$

0.36802100

1.86717200

0.26182700

$-1.85430400$

$-1.53226900$

$-0.02859300$

0.63354700

$-1.16994100$

1.55438300

$-0.84375700$

$-3.04701300$

$-4.68344100$

$-5.73689400$

$-4.69145300$
$-3.49052100$

$-1.78078700$

$-1.93767200$

$-0.77578200$

$-0.36410500$

0.73923600

0.60588400

$-0.62987900$

$-1.22104400$

1. 24185500

0.61604400

0.09776300

1.02790700

2. 25141100

0.36135300

1.85169300

2.22653200

1.84252600

1.94851600

3.64649900

4. 34315000

$-0.52363700$

$-2.09737700$

1.15329200

$-0.99328500$

1.21563600

0.97749800

1.80760100
$-0.96142800$

1.14480000

$-1.22362200$

$-1.21982100$

2.00015500

$-0.61555000$

1.02830200

3.45807500

3.48984300

$-1.03693700$

$-0.92454500$

0.57791000

$-1.88346400$

0.45390000

2.28370500

$-1.20192000$

$-1.13882300$

2.58756000

3.49167200

$-1.88962200$

$-1.79561900$

$-1.24482500$

$-0.59909500$

1.50047200

$-1.16141100$

0.35860500

0.12357300

1. 29140400 
${ }^{1} \mathrm{C} 6$

$\begin{array}{lll}-4.31449400 & 1.88424600 & -0.44065700 \\ -3.85548200 & -1.50359700 & -2.21062600 \\ -3.23678700 & -1.68304000 & -3.10588400 \\ -4.31824800 & -2.45857500 & -1.90855200 \\ -4.65045100 & -0.78420000 & -2.47214500 \\ -3.90153800 & -1.86210600 & 1.76373600 \\ -3.11335500 & -2.63406600 & 1.69922000 \\ -3.94136700 & -1.52812800 & 2.81686600 \\ -4.86514400 & -2.36386600 & 1.55915500 \\ -3.54930800 & -0.37242300 & 0.53998400\end{array}$

\subsection{0}

1.69421800

$-1.37348100$

$-0.79267500$

1.37432500

$-0.59359600$

1.13016300

1.88884500

1.19901400

2.87038700

0.46696400

$-1.19127100$

2.19972100

0.74290800

1.74969800

3.14220000

0.35549600

0.44043100

4.22389800

0.71479200

$-2.06177200$

4.66091000

1.57308000

$-1.99309100$

3.05604800

1.25002900

3.03872800

3.50956000

2.10044100

2.98149300

$-0.43529700$

$-1.24850700$

$-1.59606200$

$-0.98149400$

$-0.93268300$

1.03158500

1.07288900

$-2.40895500$

0.24522500

2. 22155600

$-0.94862400$

$-1.72441100$

1.37757800

$-0.59813000$

2. 23540300

0.39611200

$-1.74239200$

1.59124500

1.16053500

$-3.39450000$

$-2.23427100$

1.45236600

$-3.32800800$

$-3.15299500$

0.02927800

$-2.92302900$

2.64328400

$-0.18900100$

$-2.68113600$

3. 55309900

$-1.38409200$

$-0.26226700$

$-0.52015800$

1.04638800

$-2.03170900$

$-1.35833000$

$-2.95880100$

$-0.33010000$

$-0.82353200$

$-5.53186400$

1.21635900

$-1.55481000$

$-5.81397200$

0.44827400

$-2.29788600$

$-6.46660200$

1.73360900

$-1.26528300$

$-4.90761900$

1.96348200

$-2.07885400$

$-5.35717200$

$-1.18548900$

0.87231400

$-5.66219600$

$-1.94712400$

0.13169400

$-4.62838200$

$-1.66505500$

1.55181700 
$\mathrm{H}$

C

$\mathrm{H}$

$\mathrm{H}$

$\mathrm{H}$

Al

Si

0

$\mathrm{H}$

${ }^{1} \mathrm{C} 6$-7TS

0

0

0

Si

Si

0

0

$\mathrm{H}$

0

$\mathrm{H}$

0

0

0

0

0

Si

0

$\mathrm{H}$

0

H

V

Si

0

C

$\mathrm{H}$

$\mathrm{H}$

$\mathrm{H}$

C

$\mathrm{H}$

$\mathrm{H}$

$\mathrm{H}$

C

$\mathrm{H}$
$-6.25370300$

$-3.68891900$

$-2.95358800$

$-3.15008700$

$-4.41299900$

$-4.61600700$

0.61167700

0.35292700

$-0.52640600$
$-0.95708100$

1.73773800

1.24967700

2. 52452900

2. 25348900

0.44723800

2. 24585500

3.83322500

4.18423600
1.47973400

1.23869100

1.90579000

0.67954300

1.89754400

0.02599800

$-0.33087900$

$-0.57314400$

$-0.37782700$

2.10866000

$-0.09767800$

1.05147300

$-1.59234400$

2.18229600

$-0.49095800$

2.11012400

1.67357200

0.98378600

2.67724200

$-0.49795900$

$-1.41314900$

2. 61090500

0.18538900

1.52565400

3.14284700

$-0.62147000$

0.17353200

3.89307300

$-0.82564700$

$-2.45699600$

4.61374400

$-0.18519300$

$-2.50311400$

3.77771700

0.31730000

2.66510700

4.53738700

0.87168500

2.44801800

$-0.95647000$

$-0.59518000$

$-1.34696100$

$-1.13932100$

0.00409600

1.35031800

0.06730600

$-2.21948900$

0.49302800

1.49373200

$-1.59395900$

$-1.72897300$

1.37246000

$-0.63551700$

2. 21141300

$-0.13199100$

$-1.20316800$

1.80316400

$-0.60811500$

$-3.26765300$

$-1.88523500$

$-0.24227000$

$-3.46295600$

$-2.75718600$

$-0.76764000$

$-2.01002200$

3.08207000

$-0.15183600$

$-2.55043800$

3.59289000

$-1.23632300$

0.81514600

$-0.31772700$

$-0.00513400$

$-1.94865100$

$-1.12701600$

$-2.96553100$

1.28498400

$-0.07379800$

$-4.15657400$

$-1.60504400$

$-0.33702000$

$-3.46191700$

$-1.88599100$

0.47308400

$-5.08114000$

$-2.19533700$

$-0.21721500$

$-3.68217800$

$-1.92862400$

$-1.28091700$

$-3.77350500$

1.34469200

1.66570500

$-4.61769800$

0.65579000

1.87029900

$-2.89140300$

1.06128700

2. 24170200

$-4.06407900$

2.39715700

1.68457600

$-6.01881400$

1. 31804600

$-0.94095400$

$-5.91784800$

2.40231900

$-0.76501200$ 
$\mathrm{H}$

$\mathrm{H}$

Al

Si

0

$\mathrm{H}$

${ }^{1} \mathrm{C} 7$

0

0

0

Si

Si

0

0

$\mathrm{H}$

0

$\mathrm{H}$

0

0

0

0

0

Si

0

$\mathrm{H}$

0

H

V

Si

0

C

$\mathrm{H}$

$\mathrm{H}$

$\mathrm{H}$

C

$\mathrm{H}$

$\mathrm{H}$

$\mathrm{H}$

C

$\mathrm{H}$

$\mathrm{H}$

$\mathrm{H}$

Al
$-6.19219100$

$-6.93661300$

$-4.45204500$

1.55589100

2.15045200

1.54369500

$-1.57747300$

$-0.01600500$

$-2.50105900$

$-1.84964500$

$-2.94104500$

$-2.80476500$

$-2.52826700$

$-3.31671900$

$-4.44516900$

$-5.13540100$

1.69381100

0.56607200

0.12925900

$-0.43349700$

$-1.92951200$

$-0.32715000$

1.77164800

1.41707500

$-0.07052400$

$-0.76597900$

1.08909100

0.78070200

2.98682000

4.59236300

5.64946400

4.59562800

4.18896900

3.78689700

3.15223400

4.30273000

4.54201900

3.95765200

3.19687300

4.01898200

4.93228600

3.51056300
1.17419800

0.97634100

0.32195100

2.17076000

3.64424200

4.38846800

1.97963900

1.78842500

0.70479700

0.74200900

$-0.69026700$

$-0.35020700$

1.26758800

1.81947000

$-1.17492300$

$-0.50028300$

0.03341100

$-1.24649300$

$-2.11304300$

0.02638200

$-1.92377200$

$-2.30803500$

$-1.41465400$

$-1.43673100$

$-3.83286000$

$-4.48005900$

0.39340900

$-0.87886100$

0.83254400

$-1.19837000$

$-1.02963000$

$-1.66635600$

$-1.95067300$

1.14009400

1.21474900

2.10546700

0.35274400

2.06381500

2.85227200

1.86971100

2.49432500

0.43801600
$-2.02307700$

$-0.42932000$

$-0.34535700$

$-0.49888700$

$-0.89917800$

$-0.79896300$

0.79296500

$-1.39471000$

$-1.34629700$

1.86182800

$-0.56098300$

1.05813300

3.25274200

3.17842300

$-0.97829200$

$-0.99358100$

0.59336700

$-1.73315300$

0.75652900

2. 28420500

$-0.94641900$

$-0.82605400$

2.82743400

3. 72510400

$-1.35494400$

$-1.18231700$

$-1.29378700$

1.63864800

$-1.30603400$

0.46730500

0.19156400

1.46822400

$-0.23523000$

$-2.43465200$

$-3.33401200$

$-2.29104200$

$-2.60420900$

1.45264900

1.30792000

2.53935500

1.15729000

0.45056600 
0

$\mathrm{H}$

Si

${ }^{3} \mathrm{~A} 6$

$\mathrm{V}$

0

0

0

0

Si

Si

Si

0

0

0

0

$\mathrm{H}$

0

$\mathrm{H}$

0

$\mathrm{H}$

C

$\mathrm{H}$

$\mathrm{H}$

$\mathrm{H}$

C

$\mathrm{H}$

$\mathrm{H}$

$\mathrm{H}$

C

$\mathrm{H}$

$\mathrm{H}$

$\mathrm{H}$

Al

${ }^{3} \mathrm{~A} 6-7 \mathrm{TS}$

$\mathrm{V}$

0

0

0

0

Si

Si
$-2.23475700$

$-1.62753500$

$-1.57080200$

3.36146200

4.08628700

1.99133300

$-1.46933300$

$-1.66486400$

$-0.86802800$

$-0.56588900$

$-2.23340000$

$-0.09769500$

0.85353600

$-0.14289300$

1.38931900

2. 39501100

1.34796700

1.54553100

2.51145900

2.45594600

1.57942900

1.46704300

1.59186400

2.40287400

3.50869300

4.43956300

$-4.77127200$

$-5.25904900$

$-5.56345400$

$-4.09513700$

$-4.55094100$

$-3.76951300$

$-5.33234200$

$-5.01419900$

$-2.83201900$

$-2.21976000$

$-2.32484100$

$-3.73684700$

$-3.80657000$

$-0.00794200$

0.84618600

0.00107100

1.21413300

$-1.42737400$

$-0.21135700$

0.05602700

1.93263100

1.35361400

$-1.45651900$

0.06584900

1.38407400

$-0.06001000$

$-1.28717000$

1.36207300

2.62250400

3.51598400

$-2.81642700$

$-2.89288000$

0.12469700

0.09474100

$-1.67464700$

$-1.79273500$

$-1.73718000$

$-2.53600800$

1.82966400

2.56891800

1.88403400

2.14804800

$-0.18600900$

0.72081400

$-1.14143100$

$-0.17492700$

0.02858000

$-0.30651700$

$-0.93493600$

1.27477400

$-1.03764900$

$-1.84627800$

0.30266300

0.22350900

$-2.07621000$

$-1.72710100$

$-1.81344800$

$-2.33232400$

2.46297800

2. 20694000

0.42086700

1.40673900

$-0.34502300$

0.28715900

0.16485000

$-0.08027400$

$-0.61259200$

1.11764600

$-1.83927600$

$-1.78918800$

$-1.66878500$

$-2.45494400$

0.36641100

$-0.57432900$

0.61875300

$-0.47192000$

$-0.54333200$

2.39577400

$-0.79598800$

1.21251400

0.15927600

$-0.74919700$

$-1.42631100$

$-0.73864400$

$-1.31881400$

$-0.80066500$

0.11180800

1.27298800

1.67002300

$-0.33315000$

$-0.97931100$

$-0.92778600$ 


$\begin{array}{lrrr}\text { Si } & -1.41764800 & 1.71406000 & -0.31576300 \\ \text { O } & -1.60781100 & 1.28314600 & 1.28104500 \\ \text { O } & -2.42926000 & -1.16724500 & 0.66956200 \\ \text { O } & -2.45884200 & 0.72629100 & -1.18557000 \\ \text { O } & -1.75288500 & 3.30546700 & -0.49046500 \\ \text { H } & -1.59767000 & 3.68697600 & -1.36368900 \\ \text { O } & -1.62367800 & -0.63614700 & 3.25693100 \\ \text { H } & -2.47010300 & -0.34651900 & 3.62044500 \\ \text { O } & -3.37205500 & -1.79403900 & -1.85037000 \\ \text { H } & -4.30708200 & -1.64771900 & -1.65824400 \\ \text { C } & 3.10532100 & -1.95176400 & 0.99107900 \\ \text { H } & 3.23913600 & -2.85017000 & 0.38803300 \\ \text { H } & 4.02889600 & -1.51232700 & 1.39700700 \\ \text { H } & 2.20966300 & -1.85868300 & 1.60844200 \\ \text { C } & 5.44147800 & -0.09104800 & -1.06793600 \\ \text { H } & 5.58987600 & 0.59257100 & -1.92421300 \\ \text { H } & 6.30639300 & 0.05631700 & -0.39700300 \\ \text { H } & 5.48268900 & -1.12067800 & -1.46088700 \\ \text { C } & 3.24430000 & 1.77883900 & 0.97631200 \\ \text { H } & 2.41873000 & 2.33457500 & 0.49586200 \\ \text { H } & 2.83426800 & 1.40009100 & 1.92973000 \\ \text { H } & 4.05464500 & 2.48984400 & 1.20603300 \\ \text { Al } & 3.75686900 & 0.29679100 & -0.17420700\end{array}$

${ }^{3} \mathrm{~A} 6 \mathrm{~b}$

$\begin{array}{lrrr}\text { V } & -0.36685000 & -0.18195600 & 0.71098700 \\ \text { O } & -2.05217100 & -0.36982600 & 0.98027200 \\ \text { O } & -0.11523900 & -0.26566000 & -1.10499000 \\ \text { O } & 0.86800500 & -1.37386800 & 1.24339300 \\ \text { O } & 0.53913700 & 1.33651000 & 1.06777800 \\ \text { Si } & 1.40515900 & -0.10572800 & -1.73636000 \\ \text { Si } & 2.43247400 & -1.26780700 & 0.66815600 \\ \text { Si } & 2.08481100 & 1.56302200 & 0.46835700 \\ \text { O } & 2.02391000 & 1.34878900 & -1.18143100 \\ \text { O } & 2.34726800 & -1.28192100 & -1.00079600 \\ \text { O } & 2.96523300 & 0.26809600 & 1.06189500 \\ \text { O } & 2.73424000 & 3.01593000 & 0.81990000 \\ \text { H } & 2.57424000 & 3.37177600 & 1.70348500 \\ \text { O } & 1.37966400 & -0.21114800 & -3.36376500 \\ \text { H } & 2.17341600 & 0.06437900 & -3.84021500 \\ \text { O } & 3.34810900 & -2.46310500 & 1.29093900 \\ \text { H } & 4.27239700 & -2.51142100 & 1.01461600 \\ \text { C } & -3.23148600 & -1.01391700 & -1.79259600 \\ \text { H } & -3.15716500 & -2.09788900 & -1.65722000\end{array}$


${ }^{3} \mathrm{~A} 7$

V

0

0

0

0

Si

Si

Si

0

0

0

0

$\mathrm{H}$

0

$\mathrm{H}$

0

$\mathrm{H}$

C

$\mathrm{H}$

$\mathrm{H}$

$\mathrm{H}$

C

$\mathrm{H}$

$\mathrm{H}$

$\mathrm{H}$

Al

C

$\mathrm{H}$

$\mathrm{H}$

$\mathrm{H}$

${ }^{3} A 7 b$

$\mathrm{V}$

0
-4.09664800
-2.28609000
-4.96285400
-5.34283200
-5.81642800
-4.48566100
-3.67911700
-4.33074000

$-0.64031700$

$-0.45650900$

$-1.67568400$

$-1.43244900$

$-1.70569300$

$-2.66776400$

$-0.27678200$

1.72432100

$-2.35046400$

$-1.85302400$

0.79700500

1.80611900

0.10195900

0.83101800

0.31407600

$-0.01918400$
$-1.33990700$

$-1.36912200$

$-1.26993900$

0.63940100

$-1.40039700$

$-0.18233500$

1.68048200

$-0.31375000$

$-0.43858500$

1.35337700

1.22368200

$-0.52394700$

$-0.82856500$

$-0.31623000$

0.20299700

3.20937500

3.93648300

1.02677500

2. 12401600

0.71087800

0.59454800

0.96796200

0.48535600

0.67788500

2.05814200

0.49804300

$-2.45984400$

$-3.41433100$

$-2.40180200$

$-2.45414100$
$-0.03358300$

$-0.01809900$

$-1.47179600$

0.06123100

1.37105000

$-1.45999700$

0.09016700

1.40066000

$-0.05111500$

$-1.26984000$

1.38562600

2.63844500

3.47446800

$-2.80486200$

$-2.83716800$

0.17028100

0.17164800

$-1.72477200$

$-1.85812300$

$-1.87051500$

$-2.55384300$

1. 74110800

2.58308300

1.81322000

1.92046300

0.01781900

$-0.09543500$

$-0.09555900$

$-1.02253800$

0.77124000

3.96763500

$$
0.77124000
$$

$-0.59300300$

$-0.88741600$

$-0.79959200$

$-2.23810400$

$-0.41245800$

$-1.25015500$ 
${ }^{3} \mathrm{~B} 6$

0

0

0

0

Si

Si

0

0

$\mathrm{H}$

0

$\mathrm{H}$

0

0

0

0

0

Si

0.61785400
-0.82791700
0.56661200
2.00343900
0.52065900
1.92361300
2.78871500
1.46120800
1.37042700
2.86312500
2.44011800
2.91210700
3.83518200
-0.00186200
0.62545700
-3.90436100
-3.87795600
-4.93638200
-3.69771300
-2.48420100
-1.66619100
-3.41971600
-2.27781100
-2.59772400
-2.33231700

$-1.82047100$

0.47942500

0.07207900

$-1.18206500$

1.22564200

0.83096800

$-0.34784800$

$-0.00257300$

1.87314600

1.55168700

2.12617800

$-2.33552000$

$-2.13385400$

2. 31536800

2.93877700

0.36234100

1.00249400

0.38990500

$-0.67432300$

2.65897700

2.66919500

2. 92420100

3.45267400

0.92423800

$-2.68552800$
0.14328500

0.60294600

$-1.80715700$

0.81562200

1.26602000

$-1.19816000$

$-0.39879400$

1.87326900

$-0.00851200$

$-2.31317200$

$-2.96458400$

1. 51055300

1.71248600

2. 35329600

2.74175000

1. 38010600

2. 27985100

0.98817400

1.69723900

$-0.86614300$

$-1.60565400$

$-1.38787700$

$-0.12470800$

0.04743500

$-0.39174700$
$-0.91674600$

$-2.21753800$

0.63429200

$-1.26027500$

$-3.05887000$

$-2.07557600$

$-3.09288900$

$-4.55547100$

$-5.08114700$

$-2.87938700$

$-3.43164400$

0.39180600

1.20280100

$-0.67629600$

$-2.27968100$

$-1.02359000$

0.10623100
$-3.71794800$

$-1.31552000$

$-1.49110400$

$-1.89844200$

0.03929400

$-0.66353600$

$-0.02558700$

0.10022100

$-0.70917200$

$-1.18350600$

$-1.96920000$

1.35275600

0.53668900

2. 39945200

1.40321600

0.48822000

1.45196100
$-0.96239600$

$-1.42744700$

$-1.07448100$

1.08804900

$-0.94157600$

1.85984900

0.71512900

$-1.59606300$

$-1.56929300$

3.18503100

3.08698400

$-1.67585800$

0.83059000

0.52750700

$-1.42023800$

2.37080600

1.64505800 
${ }^{3} \mathrm{~B} 6-7 \mathrm{TS}$

0

0

0

0

Si

Si

0

0

$\mathrm{H}$

0

$\mathrm{H}$

0

0

0

0

0

Si

0

$\mathrm{H}$

0

$\mathrm{H}$
$-0.97141600$

$-1.45448800$

0.88830600

0.37520400

1.43770400

$-0.89280400$

$-0.89875700$

3.11706600

6.22741500

6.33162900

7.03949500

6.39001900

4.48343900

4.90872700

3.40017300

5.12795100

3.80138200

2. 91205200

3.48749400

4.55446500

4.45567000
3.73474500

3.81682400

2. 34406000

2.62872400

0.13032700

$-2.19200800$

2. 24405800

0.07517600

$-0.37966400$

0.61468700

$-0.50140700$

$-1.13259300$

1.05447600

1.86264900

1.04572200

0.59401900

$-2.10930800$

$-1.79688000$

$-2.94959700$

$-2.49292000$

$-0.64688600$
$-1.76366700$

$-2.59584500$

2.77401400

3.54106300

$-0.92529800$

$-0.62454800$

$-1.09821600$

$-1.18295800$

$-1.00778800$

$-1.47377100$

$-0.27056700$

$-1.80191500$

1.42613600

0.82186800

1.59270500

2.18219800

0.91334300

1.48672600

0.26585600

1.62215300

$-0.21904700$
$-0.10908100$

$-1.77496700$

0.98613300

$-1.02740400$

$-2.85600900$

$-2.11350600$

$-3.08089300$

$-4.25808000$

$-4.65434600$

$-2.97887100$

$-3.38527800$

0.39974200

1.05693400

$-1.12762400$

$-2.24088500$

$-1.32803500$

$-0.31927100$

$-1.39149900$

$-1.81679900$

0.05737400

$-0.65503500$
$-3.75102800$

$-1.67216100$

$-1.27484700$

$-1.89776800$

$-0.43144900$

$-0.76419600$

$-0.39079100$

$-0.65891500$

$-1.53763200$

$-1.33001400$

$-2.19964100$

1.48791100

1.03227700

2.40008700

1.00031300

0.57436400

1.71456100

3.52698400

3.45444000

2.82915800

3.08801700
$-0.64630600$

$-1.44818000$

$-0.78257400$

1.19589100

$-1.17385100$

1.76544500

0.46894300

$-1.98623100$

$-1.93337000$

3.03409600

2.93208800

$-1.64267100$

1.06282200

0.34959900

$-1.68409200$

2. 28829500

1.63340000

$-2.05730300$

$-2.92104600$

2.77579800

3.37405200 
${ }^{3} \mathrm{~B} 6 \mathrm{~b}$

$$
0
$$$$
0
$$$$
0
$$$$
0
$$$$
\text { Si }
$$$$
\text { Si }
$$$$
0
$$$$
0
$$$$
\mathrm{H}
$$$$
0
$$$$
\mathrm{H}
$$$$
0
$$$$
0
$$$$
0
$$$$
0
$$$$
0
$$

0

$\mathrm{H}$

0

H

V

V

Si

0
1.48753200

$-0.41759700$

$-1.09498400$

3.26963300

6.11488000

6.37681000

6.93715100

6.08635700

4.20289600

4.49027900

3.29448200

5.02208800

3.65366000

2.93325200

3.08661800

4.41159900

4.39912900

0.47876800

$-2.22978900$

2.11961800

0.52754800

$-0.76954700$

0.21960000

$-1.09951900$

$-1.47704600$

1.79744500

2.53796900

1.95354400

1.25084400

$-1.96640200$

$-1.44044000$

$-2.76595100$

$-2.45323500$

$-0.75314500$

$-0.57253200$

$-2.17698500$

0.65469700

$-1.23289300$

$-3.17488500$

$-2.19985600$

$-3.24491400$

$-4.64901700$

$-5.08016700$

$-2.98320700$

$-3.51454000$

0.13217200

0.96694600

$-1.13988800$

$-2.53103100$

$-1.28191200$

$-0.26885600$

$-1.52788800$

$-1.98473100$

0.32825400

$-0.25218900$

1.27675700

$-0.77923500$

$-1.29096300$

2.96122600
$-3.77973400$

$-1.64826100$

$-1.38417900$

$-1.79248300$

$-0.34357700$

$-0.56630000$

$-0.19736800$

$-0.53528500$

$-1.39174700$

$-0.99824400$

$-1.80380400$

1.30874500

0.91985600

2.44303100

1.02422200

0.73922500

1.76291500

3.45776300

3.38337400

2.88439000

3.17975900

0.32157100

$-2.23631200$

2.08035300

0.25330100
$-0.63680500$

$-0.43973700$

$-1.27464700$

$-0.63433000$

$-1.02578000$

$-1.43749900$

$-0.36716800$

$-1.87512500$

0.76399800

0.01760700

1.34835200

1.25110300

1.21510600

1.86512800

0.70447100

1.85159000

$-0.10958100$

$-0.46894600$

$-1.25410700$

$-0.81505800$

1.33089300

$-0.96043800$

1.92438100

0.68982500

$-1.63795000$

$-1.52493700$

3.29168200

3.26563300

$-1.79071700$

0.79461500

0.24515800

$-1.60192700$

2.29872900

1.48719500

$-2.19524800$

$-3.04285300$

2.51346600

3.22657500

$-0.88092800$

$-0.32585400$

$-1.35264700$

$-1.17331300$ 
5.95074000

$-2.46809600$

$-0.52619900$

4.54693000

$-2.66500100$

$-1.63011500$

3.28548000

$-1.64438500$

1.25840300

2.73619400

$-0.78808500$

1.67086900

2.72126900

$-2.33722400$

0.62474300

4.11131700

$-2.05330500$

1.84923000

4.37699400

$-0.43347800$

$-0.36253800$

5.45017300

1.00418400

0.77549300

3B7

0

0

0

0

Si

Si

0

0

$\mathrm{H}$

0

$\mathrm{H}$

0

0

0

0

0

Si

0

$\mathrm{H}$

0

$\mathrm{H}$

V

V

Si

0

C

$\mathrm{H}$

$\mathrm{H}$

$\mathrm{H}$

C

$\mathrm{H}$

$\mathrm{H}$

$-3.48233200$

$-1.80755200$

$-1.87450000$

$-0.75506200$

$-0.41172400$

0.74528600

0.58059600

$-0.69545300$

$-1.26024800$

1.24239300

0.59483700

0.03836300

1.02117300

2.20188200

0.30047900

1.88084800

2.20778200

1.75566000

1.90606200

3.65373300

4.34668700

$-0.51991700$

$-2.07364400$

1.09638000

$-0.85693200$

1.29678100

1.12027800

1.78817900

2.02949200

$-1.32720900$

$-1.47064500$

$-2.29227600$
$-1.04237700$

1.10344800

$-1.22370800$

$-1.25260400$

1.98264300

$-0.61618200$

1.02717300

3.43587500

3.44910200

$-1.02893800$

$-0.95591600$

0.59909200

$-1.88906100$

0.48467200

2.28945300

$-1.16854500$

$-1.11644300$

2. 61840200

3.50865800

$-1.81895900$

$-1.69420900$

$-1.28435400$

$-0.65423800$

1.51930600

$-1.19992000$

0.56164500

0.26710900

1.55003600

$-0.15142200$

$-2.19577700$

$-3.15069300$

$-1.88816800$ 
$\mathrm{H}$

C

$\mathrm{H}$

$\mathrm{H}$

$\mathrm{H}$

Al

${ }^{3} \mathrm{~B} 7 \mathrm{~b}$

0

0

0

0

Si

Si

0

0

$\mathrm{H}$

0

$\mathrm{H}$

0

0

0

0

0

Si

0

$\mathrm{H}$

0

H

V

V

Si

0

C

$\mathrm{H}$

$\mathrm{H}$

$\mathrm{H}$

C

$\mathrm{H}$

$\mathrm{H}$

$\mathrm{H}$

Al

$\mathrm{Cl}$

$$
\begin{array}{ll}
-4.88812900 & -0.59476800 \\
-3.92832800 & -1.93550400 \\
-3.18132300 & -2.72996100 \\
-3.90937200 & -1.71215900 \\
-4.92291100 & -2.36814800 \\
-3.54855400 & -0.34340900
\end{array}
$$

$-2.35081700$

1.67343400

1.49512400

2.75576500

1.46049700

0.60108900

$-2.02405800$

3.71851400

0.05638500

$-1.81332100$

1.42926500

1.58643800

0.08657300

2.08181800

$-0.47457300$

$-2.61305800$

1.23857900

$-1.04201700$

$-2.13081700$

$-0.16668500$

1.94197200

$-3.07272900$

$-0.36406200$

$-0.95180700$

$-3.00435100$

$-0.75153500$

0.66200600

$-2.90792400$

$-0.34762100$

3.36733700

$-3.67088700$

0.22029600

3.53355900

$-4.55794500$

$-0.61453800$

$-1.58568300$

$-5.25338700$

0.00995300

$-1.34398100$

1.54090500

$-0.38833400$

0.68179800

0.51166700

$-0.42576500$

$-2.03742400$

$-0.10742700$

$-2.27742500$

$-0.20598000$

$-0.71922900$

$-0.99889800$

2.07283500

$-2.03139500$

$-1.31351000$

$-1.78891300$

$-0.43620500$

$-1.74407600$

$-1.74747400$

1.40952500

$-2.64465600$

2.05118200

1.00575100

$-3.03729900$

2.83570000

$-0.13471400$

$-2.90211800$

$-2.86036900$

$-0.84407700$

$-3.53103800$

$-3.04366800$

1.27696700

0.78279700

$-0.93208900$

$-1.57599800$

2.22209900

0.01319100

0.50562300

$-1.60753300$

1.16330700

3.16624600

0.92159800

$-0.59105000$

4.35624800

$-1.81693700$

0.34926100

5.39713300

$-1.58301800$

0.06115300

4.40060100

$-2.60245500$

1.12329100

3.86623300

$-2.26086900$

$-0.53590700$

3.78852200

0.88059600

2.51522000

3.07226500

1.71678800

2.60299300

3.73764700

0.30678600

3.45820300

4.80100900

1.32010500

2.46505500

3.39420400

$-0.23360200$

0.96254100

4.40223500

1.73723500

$-1.44361200$ 


\begin{tabular}{|c|c|c|c|}
\hline 0 & 2.18575900 & 1.43764000 & -1.16527100 \\
\hline 0 & 0.13660200 & 2.26230800 & 0.41813000 \\
\hline 0 & 2.33962200 & 1.05221000 & 1.44740700 \\
\hline Si & 2.59007300 & -0.11071900 & -1.60899100 \\
\hline Si & 2.72830200 & -0.56029300 & 1.36820900 \\
\hline 0 & 3.13395800 & -0.83794900 & -0.21795800 \\
\hline 0 & 3.70922700 & -0.13275300 & -2.80182600 \\
\hline $\mathrm{H}$ & 4.51823000 & 0.37357700 & -2.66114000 \\
\hline 0 & 3.93938100 & -0.95704000 & 2.39329100 \\
\hline $\mathrm{H}$ & 4.78085600 & -0.49746400 & 2.28611900 \\
\hline 0 & -0.92695000 & 0.22580500 & -1.27428600 \\
\hline 0 & -0.91885900 & -0.21393700 & 1.46698800 \\
\hline 0 & -0.17617100 & -2.13538600 & -0.24647000 \\
\hline 0 & 1.28811700 & -0.92772000 & -2.18434500 \\
\hline 0 & 1.44330900 & -1.48714900 & 1.79231400 \\
\hline Si & -0.13940300 & -1.65968100 & 1.34534700 \\
\hline 0 & -1.10303200 & -2.08968200 & -2.73117900 \\
\hline $\mathrm{H}$ & -1.09854300 & -1.75595300 & -3.63738600 \\
\hline 0 & -0.88682200 & -2.75527400 & 2.30768400 \\
\hline $\mathrm{H}$ & -0.36406500 & -3.51674800 & 2.58926300 \\
\hline V & -1.22174500 & 1.11568700 & 0.26809900 \\
\hline Si & -0.24040200 & -1.23423200 & -1.62975600 \\
\hline 0 & -2.87621500 & 1.53269600 & 0.30893100 \\
\hline C & -4.46619500 & 0.06015800 & -1.96058400 \\
\hline $\mathrm{H}$ & -3.50960300 & -0.39953700 & -2.25740700 \\
\hline $\mathrm{H}$ & -5.27329800 & -0.67268900 & -2.13048100 \\
\hline $\mathrm{H}$ & -4.64596300 & 0.91629200 & -2.63812700 \\
\hline C & -3.89515400 & -1.42540600 & 0.88539700 \\
\hline $\mathrm{H}$ & -3.30911300 & -1.88281100 & 0.08290300 \\
\hline $\mathrm{H}$ & -3.35135700 & -0.99458100 & 1.73221400 \\
\hline $\mathrm{H}$ & -4.91058200 & -1.79976100 & 1.04933500 \\
\hline C & -5.91148500 & 1.23339400 & 1.03507200 \\
\hline $\mathrm{H}$ & -5.63764200 & 1.22661300 & 2.10404500 \\
\hline $\mathrm{H}$ & -6.22814800 & 2.26431800 & 0.78948000 \\
\hline $\mathrm{H}$ & -6.79136900 & 0.58012500 & 0.90345200 \\
\hline Al & -4.41147700 & 0.71125100 & -0.10896300 \\
\hline Si & 1.77399000 & 2.11276800 & 0.29829000 \\
\hline 0 & 2.40738600 & 3.61120700 & 0.48318200 \\
\hline $\mathrm{H}$ & 3.36861000 & 3.69308100 & 0.48255700 \\
\hline
\end{tabular}

${ }^{3} \mathrm{C6}-7 \mathrm{TS}$

0

0

-1.71488600
0.83883900

$-1.65508500$

$-1.40140900$

$-1.52901800$

$-0.56889400$ 
0

Si

Si

0

0

$\mathrm{H}$

0

$\mathrm{H}$

0

0

0

0

0

Si

0

$\mathrm{H}$

0

H

V

Si

0

C

$\mathrm{H}$

$\mathrm{H}$

$\mathrm{H}$

C

$\mathrm{H}$

$\mathrm{H}$

$\mathrm{H}$

C

$\mathrm{H}$

$\mathrm{H}$

$\mathrm{H}$

Al

Si

0

$\mathrm{H}$

${ }^{3} \mathrm{C} 6 \mathrm{~b}$

0

0

0

Si

Si
$-1.23034900$

$-2.82933300$

$-2.32480800$

$-3.22117000$

$-4.13814100$

$-4.57869100$

$-3.25685400$

$-3.66941600$

0.47806200

0.94025300

$-1.05245500$

$-2.19151900$

$-1.56017800$

$-0.40837600$

$-1.03646500$

$-1.40949700$

$-0.03573100$

$-0.77883400$

1.44737100

$-0.94354400$

3.23353200

6.28920200

7.14864500

6.52956000

6.23377000

4.03325000

4.43407300

3.06136600

4.76011400

4.24610100

4.30340900

3.21104900

4.92891600

4.63728000

$-0.62052800$

$-0.50438100$

0.22382000

$-2.46258300$

$-0.37433000$

$-2.41704000$

$-2.79920800$

$-2.69798400$
$-1.83511400$

$-0.43237700$

$-0.68069100$

$-0.33091200$

$-0.71520000$

$-1.56587100$

$-1.20499200$

$-2.07103600$

1.17239600

0.86729200

2.38935000

0.99045500

0.66905500

1.69507700

3.37339100

3. 28126800

2. 82999700

3.20457900

0.17715900

1.99423800

0.36983100

$-0.10489100$

$-0.19927800$

$-0.72252900$

0.94431000

1. 39901900

2. 24175100

1.50615600

0.75235700

$-2.40593500$

$-2.30095200$

$-2.71375800$

$-3.22360900$

$-0.71219100$

$-2.23530200$

$-3.87142900$

$-4.21206900$

0.53392200

1.64738800

2.14478200

0.97068000

1.65224500

$-0.74955000$

$-1.01318900$

0.25313800

1.13686400

$-1.60205400$

$-1.28984000$

1.67466900

0.32008200

$-2.23244300$

2.91464400

2.80868500

$-1.61295100$

1.17518100

0.22060300

$-1.79988200$

2.20255100

1.58962900

$-2.26049500$

$-3.14612800$

2.71613600

3.20577100

$-0.46235100$

$-1.37807700$

$-0.35645200$

$-1.05332600$

$-0.36535700$

$-1.93802800$

$-1.38784400$

1.25564800

0.69114200

1.74034800

1.76767500

0.65622800

1.75531700

0.42557000

0.37039700

$-0.22183400$

$-0.28949600$

$-0.37023800$

$-0.90414700$ 


\begin{tabular}{|c|c|c|c|}
\hline 0 & -3.18604300 & -0.86016100 & -0.47275500 \\
\hline 0 & -4.00875800 & -1.69083700 & 2.00331700 \\
\hline $\mathrm{H}$ & -4.83443300 & -1.19735500 & 2.07554800 \\
\hline 0 & -3.80863900 & 0.46329000 & -2.78392800 \\
\hline $\mathrm{H}$ & -4.69218500 & 0.74552000 & -2.51845300 \\
\hline 0 & 0.67716400 & -0.45433100 & 1.38734800 \\
\hline 0 & 0.89374200 & 0.68109600 & -1.14939900 \\
\hline 0 & 0.15443200 & -1.88189100 & -0.81681800 \\
\hline 0 & -1.49727900 & -1.99488200 & 1.30357900 \\
\hline 0 & -1.32615100 & -0.25521900 & -2.34546900 \\
\hline Si & 0.21966100 & -0.63112000 & -1.89646200 \\
\hline 0 & 0.87605900 & -3.18716200 & 1.47171300 \\
\hline $\mathrm{H}$ & 1.83666000 & -3.14662100 & 1.35941800 \\
\hline 0 & 1.14171300 & -0.98562500 & -3.20334700 \\
\hline $\mathrm{H}$ & 0.81528700 & -1.66655200 & -3.80520700 \\
\hline V & 1.02542400 & 1.11289600 & 0.60107800 \\
\hline Si & 0.08269400 & -1.90469600 & 0.84219100 \\
\hline 0 & 2.69468700 & 1.48682800 & 0.86146100 \\
\hline C & 4.14630300 & 1.13649100 & -1.83668900 \\
\hline $\mathrm{H}$ & 4.86514100 & 0.42638100 & -2.25882000 \\
\hline $\mathrm{H}$ & 3.07637700 & 0.90679100 & -1.93684200 \\
\hline $\mathrm{H}$ & 4.43808300 & 2.19178100 & -1.82027000 \\
\hline C & 5.90193300 & 1.31382600 & 0.83830300 \\
\hline $\mathrm{H}$ & 6.70521000 & 0.83077800 & 0.26056200 \\
\hline $\mathrm{H}$ & 5.95141900 & 2.40425200 & 0.69224600 \\
\hline $\mathrm{H}$ & 6.06017800 & 1.09448700 & 1.91010600 \\
\hline Al & 4.13862600 & 0.60700500 & 0.37001600 \\
\hline Si & -2.00631600 & 1.91209900 & 0.83975700 \\
\hline 0 & -2.71810100 & 3.25355700 & 1.45024400 \\
\hline $\mathrm{H}$ & -3.68218200 & 3.28954700 & 1.45061400 \\
\hline $\mathrm{Cl}$ & 3.85231300 & -1.52592700 & 0.28626800 \\
\hline \multicolumn{4}{|l|}{ C7 } \\
\hline 0 & -1.39657900 & 1.99794000 & 0.68744400 \\
\hline 0 & 0.00918000 & 1.59712800 & -1.60984600 \\
\hline 0 & -2.53467600 & 0.74413700 & -1.34839600 \\
\hline Si & -1.78282200 & 0.86926600 & 1.83500400 \\
\hline Si & -3.00714000 & -0.59813500 & -0.49889600 \\
\hline 0 & -2.81999000 & -0.19669800 & 1.10366000 \\
\hline 0 & -2.41661500 & 1.54465800 & 3.18274800 \\
\hline $\mathrm{H}$ & -3.12690200 & 2.18637600 & 3.05829100 \\
\hline 0 & -4.54402600 & -1.03532800 & -0.85161600 \\
\hline $\mathrm{H}$ & -5.21069800 & -0.33790000 & -0.82424100 \\
\hline 0 & 1.63975500 & -0.01597400 & 0.56100500 \\
\hline
\end{tabular}




$\begin{array}{lrrr}\text { O } & 0.44388300 & -1.36087600 & -1.73452000 \\ \text { O } & 0.01604800 & -2.10740700 & 0.80653800 \\ \text { O } & -0.42899500 & 0.07017900 & 2.31948900 \\ \text { O } & -2.06225200 & -1.88995300 & -0.85569200 \\ \text { Si } & -0.46877500 & -2.33089800 & -0.77227300 \\ \text { O } & 1.74741500 & -1.42213700 & 2.81752000 \\ \text { H } & 1.42402200 & -1.39844100 & 3.72708200 \\ \text { O } & -0.30223900 & -3.89525400 & -1.22666200 \\ \text { H } & -1.01388400 & -4.49591600 & -0.97192500 \\ \text { V } & 1.21552700 & 0.25454300 & -1.38988400 \\ \text { Si } & 0.72900300 & -0.88899400 & 1.64934300 \\ \text { O } & 3.10254300 & 0.63324700 & -1.33978500 \\ \mathrm{C} & 4.60003000 & -1.13964600 & 0.76974500 \\ \text { H } & 5.65898200 & -0.95251600 & 0.51383200 \\ \text { H } & 4.57853000 & -1.48339800 & 1.81886600 \\ \text { H } & 4.25912400 & -1.98855500 & 0.14943700 \\ \mathrm{C} & 4.01047900 & 0.92505800 & -2.39364500 \\ \mathrm{H} & 3.48697000 & 0.89396300 & -3.36411100 \\ \mathrm{H} & 4.43969300 & 1.93219800 & -2.26041000 \\ \mathrm{H} & 4.82587000 & 0.18283200 & -2.41184300 \\ \mathrm{C} & 3.75629300 & 2.19067300 & 1.32501200 \\ \mathrm{H} & 2.95837200 & 2.90046600 & 1.04093800 \\ \mathrm{H} & 3.76592200 & 2.14230900 & 2.42925300 \\ \mathrm{H} & 4.71897400 & 2.64324700 & 1.02391800 \\ \mathrm{Al} & 3.45833700 & 0.43098200 & 0.51837200 \\ \mathrm{O} & -2.01036700 & 3.40683100 & -1.51068200 \\ \mathrm{H} & -1.56437600 & 3.76337200 & -2.28874800 \\ \mathrm{Si} & -1.46038000 & 1.95834900 & -0.97233200\end{array}$

${ }^{3} \mathrm{C} 7 \mathrm{~b}$

0

0

0

Si

Si

0

0

$\mathrm{H}$

0

$\mathrm{H}$

0

0

0

0
$-1.57746000$

0.03411300

$-2.52878300$

$-2.06328000$

$-3.11665100$

$-3.05662200$

$-2.78307500$

$-3.49460500$

$-4.62664900$

$-5.27110700$

1.46688400

0.39435000

$-0.23575500$

$-0.75482600$
1.59073800

2.07490100

1. 31921200

0.10443000

$-0.21819600$

$-0.51528600$

0.17867900

0.82180500

$-0.37837700$

0.29600900

$-0.38122700$

$-0.56038400$

$-2.28822800$

$-0.87625500$
1.41241900

$-0.72447100$

$-1.04373600$

1.95498600

$-0.85283600$

0.78241400

3.42031900

3.52989900

$-1.45965400$

$-1.21150300$

0.65172600

$-2.03332800$

$-0.08791600$

2.13975700 
0

Si

0

$\mathrm{H}$

0

$\mathrm{H}$

V

Si

0

C

$\mathrm{H}$

$\mathrm{H}$

$\mathrm{H}$

C

$\mathrm{H}$

$\mathrm{H}$

$\mathrm{H}$

Al

0

$\mathrm{H}$

$\mathrm{Si}$

$\mathrm{Cl}$

${ }^{3} \mathrm{~A} 8$

V

0

0

0

Si

Si

Si

0

0

0

0

$\mathrm{H}$

0

$\mathrm{H}$

0

$\mathrm{H}$

C

$\mathrm{H}$

$\mathrm{H}$

C

$\begin{array}{rrr}-2.17850600 & -1.30961000 & -1.64106000 \\ -0.60194100 & -1.81039700 & -1.64195000 \\ 1.33643100 & -2.56650000 & 2.14219300 \\ 0.95079500 & -2.88651100 & 2.96773900 \\ -0.40924800 & -3.04176800 & -2.70153400 \\ -1.16582700 & -3.62828300 & -2.82694700 \\ 1.19257400 & 0.71636500 & -1.01747600 \\ 0.42819100 & -1.55743000 & 1.22471300 \\ 3.07861100 & 0.92928300 & -0.63526900 \\ 4.31489100 & -1.75908600 & 0.38500200 \\ 5.36133300 & -1.51701300 & 0.12508200 \\ 4.34565200 & -2.53135600 & 1.17321300 \\ 3.85827600 & -2.22511600 & -0.50674000 \\ 3.66248600 & 0.95681600 & 2.49105100 \\ 2.94121700 & 1.79106200 & 2.55144500 \\ 3.58592200 & 0.39114500 & 3.43749900 \\ 4.67517800 & 1.39788900 & 2.46613500 \\ 3.30924100 & -0.18136400 & 0.94428900 \\ -1.95807900 & 3.79522900 & -0.06896200 \\ -1.43539300 & 4.41231800 & -0.59599800 \\ -1.48698200 & 2.22671800 & -0.11847000 \\ 4.32312800 & 1.65753000 & -1.55255100\end{array}$

1.52808100

$-0.00138700$

$-0.35861600$

0.42078100

$-0.03484100$

$-1.79588000$

0.87529300

1.45215300

0.57444200

0.87366300

$-1.40954700$

0.64595800

$-1.21726200$

$-0.03305800$

$-1.51364800$

$-0.74670700$

1.45355800

0.89286600

$-0.75932800$

$-1.41248400$

0.94441300

$-1.55090000$

$-1.34286800$

$-0.52747300$

$-1.55059300$

1.31215800

$-0.57665900$

$-1.11577800$

0.03639000

1.70842900

$-1.29347300$

$-2.71851300$

1.77433300

$-0.74364900$

$-3.01078500$

2.51194800

$-2.04266800$

$-0.06620500$

$-2.92329800$

$-3.00188600$

$-0.16252900$

$-2.86686400$

$-1.18517900$

2.79444700

1.72144500

$-2.12315800$

2.88464500

1.93253100

3.82649100

0.68048100

$-0.17736100$

3.80871700

1.27001800

0.74542000

3.93423600

1.23982300

$-1.11491700$

3.82570800

$-0.67771200$

$-0.15713100$ 
$\mathrm{H}$

$\mathrm{H}$

${ }^{3}$ A8-9TS

V

0

0

0

Si

$\mathrm{Si}$

$\mathrm{Si}$

0

0

0

0

$\mathrm{H}$

0

$\mathrm{H}$

0

$\mathrm{H}$

C

$\mathrm{H}$

$\mathrm{H}$

C

$\mathrm{H}$

$\mathrm{H}$

${ }^{3} \mathrm{~A} 8-12 \mathrm{TS}$

V

0

0

0

$\mathrm{Si}$

$\mathrm{Si}$

$\mathrm{Si}$

0

0

0

0

$\mathrm{H}$

0

$\mathrm{H}$

0

$\mathrm{H}$
$3.93393800-1.26486900$

$3.80973400-1.23862300$

$-1.07749100$

0.78328000

$-1.18318600$

0.65063500

$-1.10135000$

$-0.20248900$

1.91041700

$-0.19930800$

0.13208200

$-0.32425900$

$-1.96747700$

$-1.52391100$

$-0.62033600$

0.36775800

1.09007500

1.40010200

0.69471600

1.40880300

$-0.84696000$

$-1.06758900$

$-0.22311100$

$-1.08461700$

1.28792400

0.52915600

0.30052300

1.83723200

2.07912300

0.49366900

$-0.30746400$

0.84104600

$-1.78320900$

0.21064700

$-0.70166300$

$-2.07472500$

2.49849800

$-0.04629400$

$-2.30210300$

3.17029800

1.87216500

2.66425600

1.37776900

2.72092100

2.47559700

1.79742300

2.49414800

$-1.65868300$

$-1.98479500$

3.26493600

$-2.01979100$

$-1.52931900$

$-3.39045700$

0.59202000

$-0.94410300$

$-3.79775500$

0.12914500

$-1.85292200$

$-3.64856800$

1.65184400

$-0.80803000$

$-3.31859700$

$-0.21161700$

0.22446000

$-3.41567000$

0.25634300

1.20768300

$-3.62796500$

$-1.25864700$

0.19018300
$-1.34232700$

$-0.52847900$

0.05374900

$-1.30713800$

0.90513000

1.49310800

0.15322300

0.67293300

1.95710400

1.21669800

$-0.00176500$

0.80204100

1.48664600

2.34609300

2.61104200

3.50380200
$-0.09059700$

1.55037200

$-1.16094400$

$-0.56038500$

1.65922100

$-1.06712100$

$-0.46717500$

1.10089000

0.54305000

$-1.42486400$

$-0.89787000$

$-0.93552000$

3.18636500

3.34125100

$-2.04947200$

$-2.01809600$
$-1.05061800$

$-0.90505800$

$-1.57787700$

0.92435200

$-0.08942300$

$-0.77244000$

1.69927800

1.47793800

$-0.76713000$

0.84572800

3. 26601500

3.79994200

$-0.13036100$

0.28129700

$-1.44827700$

$-1.08211700$ 
$-0.07868000$

$-0.53923500$

$-1.49345800$

0.13917200

0.18418400

$-0.99481000$

1.67607300

0

0

$-0.26447000$

$-1.66565800$

$-1.15113300$

1.55510800

0.43316000

$-0.48874900$

Si

$-1.01758400$

0.12105600

1.58224400

Si

$-1.44726600$

$-0.53546400$

$-1.19993600$

Si

0.33684900

1.60445700

$-0.47426600$

0

$-0.33720000$

1.57245200

1.03706900

$-2.06098500$

$-0.32238400$

0.35144600

$-0.75607700$

0.97364800

$-1.53602500$

0.98409400

3.04520100

$-0.87788500$

0.54759200

3.84603500

$-0.55992500$

$-1.78065800$

0.31676000

3.01539200

$-2.60535400$

0.81864900

3.00613600

$-2.61626900$

$-0.91179800$

$-2.28065200$

$-3.36946200$

$-0.31047100$

$-2.33557800$

3.17762900

$-1.15606100$

0.06015100

3.66847500

$-1.61227400$

$-0.81674400$

3.71808500

$-1.44591800$

0.97636100

2.97452900

0.33871500

$-0.08386000$

3.09877800

0.88501300

0.86436200

3.56147800

0.83932700

$-0.87018500$

${ }^{3} \mathrm{~A} 10$

V
0
0
0
Si
Si
Si
0
0
0
0
H

$$
\begin{array}{r}
1.53269700 \\
0.79662700 \\
0.80648100 \\
-0.10539100 \\
-0.74210600 \\
-0.73716800 \\
-1.69145100 \\
-1.79122200 \\
-0.98599700 \\
-1.79554500 \\
-2.72031500 \\
-3.55051600
\end{array}
$$

0.31721700

$-0.28936700$

$-0.80994100$

1.69170300

$-0.84454500$

$-1.32950300$

1.16731700

0.44601500

$-1.88923300$

$-0.01431900$

2.42265400

2. 39159800
$-0.09301700$

1.51092500

$-1.41618800$

$-0.42138500$

1.57212600

$-1.27935200$

$-0.23151900$

1.25695300

0.28693800

$-1.37992900$

$-0.40761800$

0.08505100 


\begin{tabular}{|c|c|c|c|}
\hline 0 & -1.07645600 & -1.54101800 & 3.01646900 \\
\hline $\mathrm{H}$ & -1.94020500 & -1.96326900 & 3.10330600 \\
\hline 0 & -1.10097700 & -2.46756600 & -2.40047300 \\
\hline $\mathrm{H}$ & -2.00368100 & -2.80958000 & -2.38780000 \\
\hline C & 2.10697600 & 2.34862600 & -0.09895600 \\
\hline $\mathrm{H}$ & 2.59233100 & 2.66075400 & -1.04024200 \\
\hline $\mathrm{H}$ & 2.75344200 & 2.62058300 & 0.75218500 \\
\hline C & 0.68247400 & 2.84638900 & 0.02892000 \\
\hline $\mathrm{H}$ & 0.40718000 & 3.07805300 & 1.07081600 \\
\hline $\mathrm{H}$ & 0.40320800 & 3.70033200 & -0.61038200 \\
\hline C & 3.88042600 & -0.39548800 & -0.31932100 \\
\hline $\mathrm{H}$ & 4.45175100 & 0.38959000 & 0.18512000 \\
\hline $\mathrm{H}$ & 3.88525300 & -0.38234900 & -1.41592700 \\
\hline C & 3.29741300 & -1.40136300 & 0.36661500 \\
\hline $\mathrm{H}$ & 2.77743900 & -2.21515000 & -0.14752900 \\
\hline $\mathrm{H}$ & 3.34054400 & -1.45272300 & 1.45813500 \\
\hline \multicolumn{4}{|c|}{ AA10-11TS } \\
\hline v & 1.40582400 & -0.61820700 & -0.21641300 \\
\hline 0 & 0.63584900 & -0.62209500 & 1.51864400 \\
\hline 0 & 0.02087800 & -1.51584600 & -1.13797000 \\
\hline 0 & 0.67058900 & 1.27872100 & -0.90466000 \\
\hline Si & -0.94104400 & -0.22831500 & 1.70539000 \\
\hline Si & -1.55593200 & -1.19196500 & -0.91558700 \\
\hline Si & -0.97321200 & 1.58985300 & -0.54686000 \\
\hline 0 & -1.16102400 & 1.33008300 & 1.07341500 \\
\hline 0 & -1.89804400 & -1.18704200 & 0.72507500 \\
\hline 0 & -1.78915500 & 0.42986200 & -1.37206000 \\
\hline 0 & -1.26195700 & 3.13651200 & -0.98416100 \\
\hline $\mathrm{H}$ & -1.99477300 & 3.59087500 & -0.54806800 \\
\hline 0 & -1.46842100 & -0.25607300 & 3.25868200 \\
\hline $\mathrm{H}$ & -0.82880700 & -0.60042400 & 3.89457500 \\
\hline 0 & -2.55630600 & -2.18603000 & -1.74740800 \\
\hline $\mathrm{H}$ & -3.49145100 & -2.15071600 & -1.50955500 \\
\hline C & 2.66213200 & 1.23467700 & 0.42839000 \\
\hline $\mathrm{H}$ & 3.68800900 & 1.61592900 & 0.42234800 \\
\hline $\mathrm{H}$ & 2.30745700 & 1.15880200 & 1.46551200 \\
\hline C & 1.77791200 & 2.10783100 & -0.43272400 \\
\hline $\mathrm{H}$ & 1.37652400 & 2.98939700 & 0.09860100 \\
\hline $\mathrm{H}$ & 2.29763500 & 2.46652000 & -1.33458100 \\
\hline C & 3.84971100 & -0.67861700 & -0.08884900 \\
\hline $\mathrm{H}$ & 4.24362700 & -0.56547200 & 0.92353300 \\
\hline $\mathrm{H}$ & 4.37645400 & -0.11030700 & -0.85874200 \\
\hline C & 3.15198200 & -1.83728900 & -0.44373600 \\
\hline
\end{tabular}


H

$\mathrm{H}$

${ }^{3} \mathrm{~A} 11$

V

0

0

0

Si

Si

Si

0

0

0

0

$\mathrm{H}$

0

$\mathrm{H}$

0

$\mathrm{H}$

C

$\mathrm{H}$

$\mathrm{H}$

C

$\mathrm{H}$

$\mathrm{H}$

C

$\mathrm{H}$

$\mathrm{H}$

C

$\mathrm{H}$

$\mathrm{H}$

${ }^{3} \mathrm{~A} 12$

V

0

0

0

Si

Si

Si

0

0

0

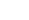

3.10954300

2.97476300

$-2.15133000$

$-1.49494200$

$-2.62286800$

0.30267100

0.87537800

$-1.24930800$

$-0.20625400$

$-0.46964800$

$-1.80167500$

0.97162000

$-0.02664900$

$-0.71237500$

$-1.76065800$

1.20352200

0.70770800

0.51120000

$-1.78395400$

$-0.82894200$

1.07989800

$-1.37450900$

0.19679500

$-1.59157000$

$-0.25986700$

1.59922700

0.65666500

$-1.21988600$

0.67694700

1.61524800

$-2.38027300$

$-0.50475400$

$-0.44714600$

$-0.91072400$

1.65739300

$-0.85772400$

0.16083800

3.05362500

1.26450200

$-0.48153100$

3.77356800

1.21955900

$-2.98471100$

$-1.37770700$

2.04635800

$-2.74143500$

$-2.09397800$

2.64648700

$-2.14737500$

0.46340900

$-3.00770000$

$-3.05876400$

0.77729400

$-2.94965600$

3.66045700

0.58765000

0.35290500

4. 50904900

1.26096600

0.14063600

3.66643200

0.40842400

1.44266500

2.40527500

1.36867600

0.00189900

2.43613200

2.37223600

0.45030200

2. 29364100

1.47699200

$-1.09119000$

3.85552300

$-0.73323800$

$-0.40736900$

4.89238500

$-1.07775800$

$-0.22851800$

3.79908700

$-0.52580600$

$-1.49306200$

2.85031800

$-1.82946700$

$-0.03958000$

3.05427800

$-2.76329200$

$-0.59874000$

2.92345900

$-2.07597900$

1.04271500

1.48579200

$-0.87734700$

0.06536400

0.67497100

$-0.38109100$

1.66811000

0.12232200

$-1.76417400$

$-0.84052300$

1.16127200

0.96640200

$-0.89939500$

$-0.85183700$

0.20530100

1.66605400

$-1.39290600$

$-1.14600000$

$-0.82473400$

$-0.43620800$

1.57907300

$-0.83106900$

$-0.83547500$

1.64469400

0.76572000

$-1.83133400$

$-0.80941000$

0.75978300

$-1.32708800$

0.39126900

$-1.53729600$ 
0

$\mathrm{H}$

0

$\mathrm{H}$

0

$\mathrm{H}$

C

$\mathrm{H}$

$\mathrm{H}$

C

$\mathrm{H}$

$\mathrm{H}$

${ }^{3} \mathrm{~A} 13$

$\mathrm{V}$

0

0

0

Si

Si

Si

0

0

0

0

$\mathrm{H}$

0

$\mathrm{H}$

0

$\mathrm{H}$

C

$\mathrm{H}$

$\mathrm{H}$

C

$\mathrm{H}$

$\mathrm{H}$

C

$\mathrm{H}$

$\mathrm{H}$

C

$\mathrm{H}$

$\mathrm{H}$
$-0.41112600$

$-1.07611900$

$-1.43669700$

$-2.39166800$

$-2.47102700$

$-3.40449200$

4.17863200

5.27337000

3.66914200

3.50643700

1.92117200

4.09172900

1.48606700

0.79356300

0.40985700

0.04117300

$-0.79486000$

$-1.16568100$

$-1.59496000$

$-1.65898600$

$-1.33390100$

$-2.00239000$

$-2.39718700$

$-3.21546300$

$-1.09134600$

$-2.00261100$

$-1.81021100$

$-2.73639900$

3.06881600

3.82259800

2.48101500

2.88833100

0.45544100

3.52749400

2.65084500

2. 58447900

1.94234000

3.58072500

3.67276800

4. 32891900
3.01844300

3.67905200

0.45043100

0.56279900

$-2.11359000$

$-1.88027300$

0.22130100

0.32539600

1.03914300

$-0.82643500$

1.53273000

$-1.61006900$

0.06772000

$-0.18577600$

$-1.00799200$

1.71076300

$-0.46204900$

$-1.26648500$

1.45623500

0.93793800

$-1.59330100$

0.19208600

2.85221200

3.00427400

$-0.91814100$

$-1.15310800$

$-2.44857600$

$-2.66933100$

2. 52116500

3. 31345400

2. 58786000

1. 52049000

2.57934900

1.51232200

$-2.17746300$

$-2.62834100$

$-2.53562600$

$-1.24866500$

$-0.83349900$

$-0.92961700$
$-1.59253200$

$-1.35871800$

3.17224000

3.25856800

$-1.57992300$

$-1.50086300$

0.22159600

0.17396600

0.75625600

$-0.28829000$

$-1.10630000$

$-0.79907500$

${ }^{3} \mathrm{~A} 13-14 \mathrm{TS}$

$-0.30873000$

1.41150800

$-1.41675500$

$-0.66243300$

1.67167900

$-1.08156200$

$-0.29670700$

1. 27174200

0.55779700

$-1.26983300$

$-0.56049800$

$-0.07022700$

3.21614600

3.43146200

$-2.01295400$

$-1.85346600$

0.15163500

0.02737000

1.08020300

$-0.72938700$

$-0.53973200$

$-1.63008200$

$-0.04243500$

$-1.03607600$

0.70943500

0.25491300

1.26399600

$-0.47594900$ 
$1.44555300-0.29599700$

$-0.39199100$

0

0.82541000

$-0.14726700$

1.34757700

0

0.13359100

$-1.36099800$

$-1.23939400$

0

0.13185500

1.55084800

$-1.03482900$

Si

$-0.76950100$

$-0.15398900$

1.72828200

Si

Si

$-1.42955500$

$-1.35343800$

$-0.80526400$

$-1.47921500$

1.50659400

$-0.51147600$

$-1.45546000$

1.27045000

1.12733600

$-1.54087500$

$-1.36572800$

0.87324100

$-2.08851600$

0.15329900

$-1.22003300$

$-2.19166700$

2.91577800

$-0.93434200$

$-2.99336700$

3.16823700

$-0.45801300$

$-0.99946200$

$-0.28711500$

3. 34452200

$-1.90211500$

$-0.46388300$

3.63767000

$-2.29214100$

$-2.56816300$

$-1.48656400$

$-3.23513600$

$-2.59595100$

$-1.28236100$

3.27121800

2.08293900

0.10113600

3.62435100

3.04186800

$-0.29881400$

3.08564800

2.04231000

1.18112300

3.08687600

0.99734100

$-0.68101300$

0.64150100

2. 34290200

$-0.80109800$

3.33653000

1.07112700

$-1.75448200$

2.85266300

$-1.86287200$

$-0.06923800$

2.91798600

$-2.48593800$

$-0.96920600$

2.47275800

$-2.35833900$

0.82940200

3.72308500

$-0.74845200$

0.06629900

3.95362700

$-0.39910000$

1.07786300

4.56133300

$-0.64621200$

$-0.63109400$

${ }^{3} \mathrm{~A} 14$

$\mathrm{V}$

0.83714900

$-1.26012600$

$-0.06572500$

0.21474500

$-0.72427800$

1.61068000

$-0.71131300$

$-1.74943900$

$-1.00109900$

0.96584600

0.66236700

$-0.89936800$

$-1.12964500$

0.20341400

1.69245200

$-2.02560600$

$-0.79030800$

$-0.87079800$

$-0.44005100$

1.61904800

$-0.71928900$

$-0.78472200$

1.66145200

0.89440400

$-2.33678700$

$-0.47949300$

0.75102400

$-1.60332200$

0.73872600

$-1.47594800$

$-0.09586200$

3.07740500

$-1.36224100$

$-0.64503400$

3.82938700

$-1.10499700$

$-1.61750000$

0.46907700

3.23088800

$-2.50762400$

0.82319200

3.35035400 


$\begin{array}{lrrr}\text { O } & -3.33343200 & -1.39790700 & -1.64169400 \\ \mathrm{H} & -4.17859500 & -0.96071400 & -1.47900600 \\ \mathrm{C} & 3.67745600 & 1.57105100 & 0.15398700 \\ \mathrm{H} & 3.86460300 & 2.48209200 & -0.42361000 \\ \mathrm{H} & 3.27435100 & 1.69636100 & 1.16602800 \\ \mathrm{C} & 3.97088600 & 0.35500300 & -0.33411500 \\ \mathrm{H} & 1.84856900 & 1.07287200 & -0.74265800 \\ \mathrm{H} & 4.37265400 & 0.27915000 & -1.35413800 \\ \mathrm{C} & 2.79507400 & -1.91396700 & -0.32730600 \\ \mathrm{H} & 3.02563200 & -1.98639400 & -1.40956300 \\ \mathrm{H} & 2.89904200 & -2.93419100 & 0.09233400 \\ \mathrm{C} & 3.77683900 & -0.95241300 & 0.38167100 \\ \mathrm{H} & 3.44942200 & -0.76026400 & 1.42002000 \\ \mathrm{H} & 4.77403200 & -1.43007900 & 0.46522700\end{array}$

${ }^{3} \mathrm{~B} 8$

0

0

0

0

Si

Si

0

0

$\mathrm{H}$

0

$\mathrm{H}$

0

0

0

0

0

Si

0

$\mathrm{H}$

0

$\mathrm{H}$

V

V

Si

C

$\mathrm{H}$

$\mathrm{H}$

C
$-1.85207000$

$-1.68817800$

0.56545800

$-1.67652300$

$-1.94879300$

$-1.92286100$

$-2.27567700$

$-3.20054100$

$-3.43836700$

$-3.17525800$

$-2.96408000$

1.74659500

1.82221700

0.90258300

$-0.59227000$

$-0.53939100$

0.98711500

1.69531100

1.53647400

1.66092200

2. 39216200

1.90432200

$-1.17107200$

0.95263600

4.16675700

4.55616500

3.99477300

4.00213800
3.53108600

1.11692200

2. 28565100

1.14408300

$-0.52036200$

$-0.50054700$

$-1.06426400$

$-0.76469800$

$-1.68065700$

$-0.83917800$

$-0.95070100$

$-0.09531700$

$-0.06950100$

$-2.16440800$

$-1.28570100$

$-1.18556300$

$-1.49068000$

$-2.64900800$

$-2.56436600$

$-2.54162700$

$-3.07492700$

1.04778100

2.12361900

$-1.54030200$

1.52502800

0.51164500

2.11598200

2.05641400
0.03014100

1.39166500

0.02609300

$-1.36342800$

1.50989900

$-1.53342400$

$-0.02237900$

2.53316600

2.72537100

$-2.52813300$

$-3.46378700$

1.44568400

$-1.41769500$

$-0.03356500$

2.04626000

$-2.09965300$

$-1.54926300$

2.45609400

3.40461200

$-2.61319400$

$-2.27783900$

0.02208500

0.02298200

1.49931900

0.66740400

0.81230800

1.57501400

$-0.56960000$ 
H

$\mathrm{H}$

${ }^{3}$ B8-9TS-01

0

0

0

0

Si

Si

0

0

$\mathrm{H}$

0

$\mathrm{H}$

0

0

0

0

0

Si

0

$\mathrm{H}$

0

$\mathrm{H}$

V

V

Si

C

$\mathrm{H}$

$\mathrm{H}$

C

$\mathrm{H}$

$\mathrm{H}$

${ }^{3}$ B8-9TS-02

0

0

0

0

Si

Si

0

0

0
3.69211700

4.25383600

3.09966100

1.49116500

$-0.70555200$

$-1.47338800$

$\begin{array}{rrrr}-0.70491700 & 3.76265300 & 0.31981300 \\ 0.41821000 & 1.83817000 & -1.28814700 \\ -1.97282600 & 1.34328100 & 0.31039200 \\ 0.67766600 & 1.64386200 & 1.43005700 \\ 1.65585100 & 0.75318400 & -1.63937000 \\ 1.96289700 & 0.55985700 & 1.38451800 \\ 2.44044000 & 0.53264800 & -0.19742200 \\ 2.65191300 & 1.32684500 & -2.79980400 \\ 3.13673700 & 2.13882900 & -2.60436000 \\ 3.20486500 & 1.08229700 & 2.30890600 \\ 3.31731900 & 0.64542300 & 3.16284300 \\ -1.45569500 & -1.17148100 & -1.26767200 \\ -1.15738900 & -1.49740200 & 1.50862600 \\ 0.67199800 & -2.42679600 & -0.20862000 \\ 1.02923500 & -0.65246800 & -2.18304500 \\ 1.44340800 & -0.90926900 & 1.88095000 \\ 0.37445700 & -2.08098400 & 1.38969700 \\ -0.14276000 & -3.00515900 & -2.74616600 \\ 0.63197500 & -3.16744500 & -3.29893800 \\ 0.69771100 & -3.37731700 & 2.34389000 \\ 0.23375200 & -4.19499800 & 2.12565100 \\ -2.23867300 & -0.60179900 & 0.28817000 \\ -0.43494100 & 2.22816500 & 0.19947900 \\ 0.00715600 & -1.83264100 & -1.60942100 \\ -3.65439700 & 1.60207100 & 0.05567800 \\ -3.58911300 & 2.13911600 & -0.89634900 \\ -3.82871800 & 2.26393800 & 0.91079900 \\ -4.23437500 & 0.28215500 & 0.05988200 \\ -4.84901100 & 0.02565500 & 0.93483500 \\ -4.64710600 & -0.07303700 & -0.89379000\end{array}$

2. 39821500

$-3.08210800$

$-0.97403700$

1.45561900

$-1.47542500$

1.09481300

$-0.13592500$

$-2.16321700$

$-1.21060500$

2.09182500

$-0.34345600$

$-1.33926400$

1.49604200

$-0.04232100$

1.92018300

2.08669200

1.22353000

$-0.77245100$

2.04254700

1.12369800

0.87740000

2.46921100

$-0.20341200$

3.22386500 


$\begin{array}{lrrr}H & 2.70975300 & 0.60181100 & 3.69926900 \\ \text { O } & 3.43274000 & 2.05084000 & -1.19493600 \\ H & 3.44388700 & 2.43588900 & -2.08044500 \\ \text { O } & -1.93766100 & -0.69496000 & 0.79244400 \\ \text { O } & -1.51517000 & 0.54546900 & -1.80751200 \\ \text { O } & -1.21970900 & 1.87253000 & 0.49413000 \\ \text { O } & -0.03748600 & 0.37717000 & 2.38685100 \\ \text { O } & 0.74138300 & 1.98701900 & -1.33564600 \\ \text { Si } & -0.89430000 & 1.91941600 & -1.14217100 \\ \text { O } & -2.66371600 & 1.03127400 & 2.65646300 \\ \text { H } & -2.50009300 & 1.70745800 & 3.32591700 \\ \text { O } & -1.49013800 & 3.28919900 & -1.81947700 \\ \text { H } & -2.43954300 & 3.30589400 & -1.99116800 \\ \text { V } & -1.65401700 & -1.12685700 & -1.07646500 \\ \text { V } & 1.47464200 & -1.86257500 & -0.64824000 \\ \text { Si } & -1.45508600 & 0.67734900 & 1.60598100 \\ \text { C } & -3.66405000 & -1.88934500 & -0.72301500 \\ \text { H } & -3.61912800 & -2.97813300 & -0.86157300 \\ \text { H } & -4.31274900 & -1.35931700 & -1.43337000 \\ \text { C } & -3.63332600 & -1.40410100 & 0.61721800 \\ \text { H } & -4.18358900 & -0.49717500 & 0.88285100 \\ \text { H } & -3.50940100 & -2.10744700 & 1.44400900 \\ & & & \end{array}$

${ }^{3}$ B8 - 12TS-01

$\begin{array}{lrrr}\text { C } & -4.26221800 & 2.28961500 & 0.18604800 \\ H & -5.22987000 & 2.79926600 & 0.07804600 \\ H & -3.52398500 & 2.81232500 & 0.81039900 \\ \mathrm{C} & -3.97845700 & 1.10346600 & -0.38659200 \\ \mathrm{H} & -3.09729200 & 0.40134900 & 0.55192900 \\ \mathrm{H} & -4.75851000 & 0.60984900 & -0.98584600 \\ \mathrm{O} & 1.71517500 & 3.49687800 & -0.75088200 \\ \mathrm{O} & 1.89150800 & 0.79662600 & -1.37605700 \\ \mathrm{O} & -0.62716800 & 2.14701600 & -0.96163700 \\ \mathrm{O} & 1.23437000 & 1.57269300 & 1.18079100 \\ \mathrm{Si} & 2.25651700 & -0.77083400 & -0.93913700 \\ \mathrm{Si} & 1.55597700 & 0.09893400 & 1.88843900 \\ \mathrm{O} & 2.28517100 & -0.81174600 & 0.71149400 \\ \mathrm{O} & 3.72942100 & -1.23927200 & -1.47465400 \\ \mathrm{H} & 3.82268000 & -1.40338500 & -2.42144400 \\ \mathrm{O} & 2.47703700 & 0.22708600 & 3.23192100 \\ \mathrm{H} & 3.34112600 & 0.64702200 & 3.13376800 \\ \mathrm{O} & -1.36752500 & -0.69771100 & -1.76977300 \\ \mathrm{O} & -1.99786100 & 0.15336900 & 0.94491600 \\ \mathrm{O} & -0.79706400 & -2.17595000 & 0.39634800\end{array}$


0

0

Si

0

$\mathrm{H}$

0

$\mathrm{H}$

V

V

Si

${ }^{3}$ B8-12TS-02

C

$\mathrm{H}$

$\mathrm{H}$

C

$\mathrm{H}$

$\mathrm{H}$

0

0

0

0

Si

Si

0

0

$\mathrm{H}$

0

$\mathrm{H}$

0

0

0

0

0

Si

0

$\mathrm{H}$

0

$\mathrm{H}$

V

V

Si
1.07715700

0.15203900

$-1.22307300$

$-1.02114800$

$-0.74767300$

$-2.23961400$

$-1.87386400$

$-1.89177100$

1.05105600

$-0.53542900$

$-1.77002800$

$-0.61875200$

$-1.15405200$

$-3.41907800$

$-3.56350900$

$-1.84927300$

$-2.51439200$

0.82084000

2.10203900

$-2.02176900$
$-1.51185700$

2.35265900

1.62199500

$-1.93362900$

$-2.84800800$

2.69580100

3.29306200

$-0.93107500$

$-0.50710500$

$-1.22944200$

\begin{tabular}{|c|c|c|}
\hline 4.49950100 & 1.92404000 & 0.45520100 \\
\hline 5.54438200 & 2.26420600 & 0.48570700 \\
\hline 3.94244000 & 2.04066000 & 1.39585500 \\
\hline 3.91707800 & 1.40242500 & -0.64198100 \\
\hline 2.51040300 & 1.96607100 & -0.60029500 \\
\hline 4.51493000 & 1.33017800 & -1.56394300 \\
\hline-0.44887000 & 3.84195000 & -0.39629600 \\
\hline-0.97159400 & 1.72970200 & 1.28847600 \\
\hline 1.39373900 & 1.79489000 & -0.37679200 \\
\hline-1.28115600 & 1.42505100 & -1.41439600 \\
\hline-1.76258200 & 0.30678000 & 1.70246000 \\
\hline-2.15949000 & -0.00238400 & -1.30056900 \\
\hline-2.49514300 & -0.19619200 & 0.30353000 \\
\hline-2.83590100 & 0.53631700 & 2.91150000 \\
\hline-3.54702900 & 1.17150300 & 2.75804000 \\
\hline-3.58082000 & 0.09648500 & -2.10108700 \\
\hline-3.55154300 & 0.01123200 & -3.06266000 \\
\hline 1.86704100 & -0.82362200 & 1.23383900 \\
\hline 1.47155600 & -1.04241900 & -1.58882900 \\
\hline-0.01225900 & -2.40072000 & 0.18447000 \\
\hline-0.67584800 & -0.79786300 & 2.21808800 \\
\hline-1.23105900 & -1.22592300 & -1.87414200 \\
\hline 0.16168600 & -2.01814300 & -1.42302300 \\
\hline 0.94514200 & -2.88146400 & 2.70018900 \\
\hline 0.23615600 & -3.17934400 & 3.28383700 \\
\hline 0.35564100 & -3.35184300 & -2.35513500 \\
\hline-0.38804300 & -3.96607400 & -2.39641200 \\
\hline 2.26832600 & 0.00927000 & -0.33229200 \\
\hline-0.30300600 & 2.29722300 & -0.23939800 \\
\hline 0.54843800 & -1.73492200 & 1.59985300 \\
\hline
\end{tabular}

${ }^{3} \mathrm{~B} 9-01$ 


\begin{tabular}{lrrr} 
O & 2.48252800 & -2.95583200 & -1.14735600 \\
O & 1.52629300 & -1.51066500 & 1.02484500 \\
O & -0.08021800 & -2.10328800 & -1.29754900 \\
O & 2.08117200 & -0.20482400 & -1.34177000 \\
Si & 1.46109900 & -0.12809700 & 1.93691500 \\
Si & 2.04073000 & 1.31897200 & -0.67241400 \\
O & 1.99197400 & 1.10360300 & 0.96819200 \\
O & 2.30042400 & -0.23384300 & 3.33511700 \\
H & 3.24008300 & -0.44675600 & 3.26756300 \\
O & 3.37431000 & 2.19602100 & -1.02838900 \\
H & 3.34931700 & 2.70891100 & -1.84617400 \\
O & -1.98270200 & -0.75259100 & 0.67381900 \\
O & -1.53276000 & 0.66584800 & -1.82776600 \\
O & -1.29747400 & 1.80015600 & 0.58182900 \\
O & -0.10435000 & 0.17240600 & 2.37873900 \\
O & 0.68673100 & 2.09706800 & -1.18967600 \\
Si & -0.95157400 & 1.98680100 & -1.04564800 \\
O & -2.70443300 & 0.83306600 & 2.70670300 \\
H & -2.46169200 & 0.90474100 & 3.63887300 \\
O & -1.55611900 & 3.40420600 & -1.60334900 \\
H & -2.50685100 & 3.43309600 & -1.76689700 \\
V & -1.63231400 & -1.09625600 & -1.30966500 \\
V & 1.52316600 & -1.78727800 & -0.74279700 \\
Si & -1.50543100 & 0.56404200 & 1.62987200 \\
C & -3.26370900 & -1.53651500 & 0.70056900 \\
H & -4.04059300 & -0.87044800 & 1.10182500 \\
H & -3.09524200 & -2.36663500 & 1.40129100 \\
C & -3.45195200 & -1.95878700 & -0.73716100 \\
H & -3.46927000 & -3.04976300 & -0.89003500 \\
H & -4.32192600 & -1.49474400 & -1.22951200 \\
& & & \\
\hline
\end{tabular}

3B9-02

$\begin{array}{lrrr}0 & 2.81654900 & 2.64490000 & 0.05281800 \\ 0 & 1.82289900 & 0.49397600 & -1.35380000 \\ 0 & 0.08416300 & 2.37556900 & 0.02803000 \\ 0 & 1.79792700 & 0.46716900 & 1.38711200 \\ \text { Si } & 1.53756700 & -1.15187700 & -1.52431900 \\ \text { Si } & 1.49601700 & -1.18893600 & 1.52643300 \\ 0 & 1.68980300 & -1.78136300 & 0.00018900 \\ 0 & 2.66024500 & -1.73698900 & -2.55482100 \\ \text { H } & 2.49211800 & -2.60355300 & -2.94676300 \\ 0 & 2.57562200 & -1.89995600 & 2.52244700 \\ \text { H } & 2.29847100 & -2.03399400 & 3.43797100 \\ 0 & -1.86057800 & 0.42878400 & -1.49243500\end{array}$




\begin{tabular}{lrrr}
0 & -1.96471000 & 0.41470000 & 1.42335200 \\
\hline & -1.66550100 & -1.81746000 & -0.01597800 \\
\hline & 0.01836000 & -1.41040300 & -2.07332400 \\
Si & -0.03322800 & -1.38337700 & 2.06846300 \\
\hline & -1.58565500 & -1.18122600 & 1.51882200 \\
\hline & -2.57462500 & -2.02844100 & -2.49914800 \\
$H$ & -2.63763000 & -1.73806000 & -3.41769000 \\
V V V Si & -2.51959200 & -2.02598300 & 2.56946300 \\
C & -3.47156800 & -1.99996000 & 2.41228500 \\
$H$ & -1.74218900 & 1.52725400 & -0.02248600 \\
$H$ & 1.69274500 & 1.56586300 & 0.02918200 \\
$C$ & -1.54486700 & -1.18289000 & -1.53992700 \\
$H$ & -0.43004200 & 3.79781500 & 0.02777400 \\
$H$ & 0.02405700 & 4.27010100 & -0.85519200 \\
\hline & -0.02419200 & 4.25180400 & 0.94319200 \\
\hline & -1.92468800 & 3.63622600 & -0.01348900 \\
\hline & -2.44265900 & 4.01611500 & 0.88103700 \\
\hline & -2.39455400 & 4.03162400 & -0.92762300
\end{tabular}

${ }^{3}$ B10-01

0

0

0

0

Si

Si

0

0

$\mathrm{H}$

0

$\mathrm{H}$

0

0

0

0

0

Si

0

$\mathrm{H}$

0

H

V

V

Si
$-1.23503200$

$-1.80759800$

0.81167700

$-1.35860500$

$-2.36778200$

$-1.91595900$

$-2.58202700$

$-3.72958400$

$-4.46526700$

$-3.02962900$

$-3.00190300$

1.34134900

1.82201500

0.24255900

$-1.24888300$

$-0.68306500$

0.66791600

0.74565100

0.09138200

1.11411100

1.92584300

2.08313900

$-0.87095000$

0.23457200
$-3.30693100$

$-0.58855600$

$-1.65460800$

$-1.91245800$

0.79445100

$-0.67496300$

0.44234200

1.37193100

0.75295800

$-1.24915000$

$-0.87413100$

1.20298300

0.01640100

2.15421700

2.00276900

0.03184000

0.93039200

3.81169500

4.39276200

1.56304300

2.08493300

$-0.60373300$

$-1.95714600$

2.33356300
$-1.78826100$

$-1.75999400$

$-1.19658200$

0.63820100

$-1.04208800$

1.59274800

0.56115000

$-1.74194100$

$-1.83303600$

2.64637900

3.53569400

$-1.14692500$

1.40099300

1.05629100

$-1.18435600$

2.42079300

2.11327100

$-1.05340200$

$-1.46205600$

3. 56101500

3.56806900

$-0.32391300$

$-1.07972600$

$-0.57053900$ 


$\begin{array}{lrrr}\mathrm{C} & 2.53429600 & 1.40038300 & -2.01028300 \\ \mathrm{H} & 2.88158800 & 2.43421100 & -1.86491000 \\ \mathrm{H} & 2.19542400 & 1.27784800 & -3.04972000 \\ \mathrm{C} & 3.49024600 & 0.32255100 & -1.55440100 \\ \mathrm{H} & 3.84540900 & -0.34003200 & -2.36019200 \\ \mathrm{H} & 4.35019200 & 0.70317900 & -0.97839400 \\ \mathrm{C} & 3.84130100 & -2.17548300 & 0.51869400 \\ \mathrm{H} & 4.50008000 & -2.34155900 & -0.33934000 \\ \mathrm{H} & 4.20031100 & -1.49388700 & 1.29712700 \\ \mathrm{C} & 2.68558300 & -2.85491700 & 0.66198600 \\ \mathrm{H} & 2.05351200 & -2.72634300 & 1.54656500 \\ \mathrm{H} & 2.34855900 & -3.57727400 & -0.08647600\end{array}$

3B10-02

$\begin{array}{lrrr}\text { O } & -1.89655100 & 3.45062500 & -0.07504100 \\ \text { O } & -1.84431200 & 1.10270200 & 1.35977600 \\ \text { O } & 0.49466300 & 2.17115200 & -0.02397800 \\ \text { O } & -1.81572000 & 1.03945000 & -1.39195900 \\ \text { Si } & -2.17223000 & -0.52655400 & 1.54472000 \\ \text { Si } & -2.16976500 & -0.60087300 & -1.50800200 \\ \text { O } & -2.52967200 & -1.08767700 & 0.02575600 \\ \text { O } & -3.43662200 & -0.67256500 & 2.57013100 \\ \text { H } & -3.56891500 & -1.53678900 & 2.98016700 \\ \text { O } & -3.47570600 & -0.87545000 & -2.45267700 \\ \text { H } & -3.32872900 & -0.86870500 & -3.40712800 \\ \text { O } & 1.48890500 & -0.12351900 & 1.48407100 \\ \text { O } & 1.52206000 & -0.14390100 & -1.48856200 \\ \text { O } & 0.57679800 & -2.17306700 & 0.00238400 \\ \text { O } & -0.85175300 & -1.31426500 & 2.11614600 \\ \text { O } & -0.84274200 & -1.36373900 & -2.09713800 \\ \text { Si } & 0.71518700 & -1.55775800 & -1.53747400 \\ \text { O } & 1.44478000 & -2.69262800 & 2.44900200 \\ \text { H } & 1.53541300 & -2.48320000 & 3.38708600 \\ \text { O } & 1.51151400 & -2.61542500 & -2.51434300 \\ \text { H } & 1.10367300 & -3.48322500 & -2.62807200 \\ \text { V } & 2.13228300 & 0.78237200 & -0.00129900 \\ \text { V } & -1.28052800 & 2.01860800 & -0.03482900 \\ \text { Si } & 0.69051000 & -1.55036100 & 1.53095500 \\ \text { C } & 1.37695200 & 3.36558900 & -0.02148400 \\ H & 1.11082000 & 3.94591200 & 0.87515000 \\ H & 1.11261200 & 3.94816700 & -0.91727900 \\ \text { C } & 2.77230500 & 2.79770000 & -0.01724600 \\ H & 3.34965600 & 3.03389600 & -0.92631900 \\ \text { H } & 3.36039700 & 3.06819600 & 0.87469000\end{array}$


C

$\mathrm{H}$

$\mathrm{H}$

C

$\mathrm{H}$

$\mathrm{H}$

${ }^{3}$ B10-11TS-01

\begin{tabular}{|c|c|c|c|}
\hline 0 & 0.44263100 & -3.83869800 & -0.34647200 \\
\hline 0 & 1.00764600 & -1.65713300 & 1.32712400 \\
\hline 0 & -1.22160400 & -1.73926400 & -0.46156700 \\
\hline 0 & 1.49013800 & -1.48475200 & -1.40677000 \\
\hline Si & 1.93185700 & -0.33333700 & 1.69169200 \\
\hline Si & 2.39075000 & -0.09626500 & -1.30750000 \\
\hline 0 & 2.72685300 & 0.10981100 & 0.31069700 \\
\hline 0 & 2.95476000 & -0.58997900 & 2.94438900 \\
\hline $\mathrm{H}$ & 3.53803200 & -1.35654200 & 2.87903300 \\
\hline 0 & 3.76330600 & -0.18800600 & -2.19773200 \\
\hline $\mathrm{H}$ & 4.21592500 & -1.04066300 & -2.19751500 \\
\hline 0 & -1.54717900 & 0.83481400 & 1.15859200 \\
\hline 0 & -1.15654100 & 1.02107400 & -1.66374700 \\
\hline 0 & 0.21814600 & 2.47610200 & 0.08332400 \\
\hline 0 & 0.95015600 & 0.90759600 & 2.17724000 \\
\hline 0 & 1.55958900 & 1.20830300 & -1.85177200 \\
\hline Si & 0.13690200 & 1.98956100 & -1.52408500 \\
\hline 0 & -0.76052800 & 2.93482700 & 2.60396000 \\
\hline $\mathrm{H}$ & -0.11523000 & 3.29864200 & 3.22408500 \\
\hline 0 & 0.02995900 & 3.30967300 & -2.49210400 \\
\hline $\mathrm{H}$ & 0.86659600 & 3.64308300 & -2.83989400 \\
\hline V & -2.18317900 & -0.09827600 & -0.58755100 \\
\hline V & 0.37485100 & -2.27299700 & -0.24727900 \\
\hline Si & -0.22186400 & 1.84219000 & 1.51431300 \\
\hline$C$ & -2.07596700 & -0.19482000 & 2.05920200 \\
\hline $\mathrm{H}$ & -2.24064300 & 0.25634300 & 3.05411700 \\
\hline $\mathrm{H}$ & -1.32416300 & -0.99353100 & 2.14554600 \\
\hline C & -3.35131800 & -0.65706900 & 1.39032700 \\
\hline $\mathrm{H}$ & -3.65126500 & -1.63280800 & 1.79027300 \\
\hline $\mathrm{H}$ & -4.15165000 & 0.07909400 & 1.56169100 \\
\hline C & -4.23700500 & -1.39671400 & -0.51752300 \\
\hline $\mathrm{H}$ & -5.15670700 & -1.20769200 & 0.03701800 \\
\hline $\mathrm{H}$ & -3.84237700 & -2.41319700 & -0.43971200 \\
\hline C & -3.93572700 & -0.63271400 & -1.65441500 \\
\hline $\mathrm{H}$ & -3.37801300 & -1.10260600 & -2.47889600 \\
\hline $\mathrm{H}$ & -4.55456700 & 0.23204100 & -1.92335800 \\
\hline
\end{tabular}
4.58464000
0.28701800
0.07682900
4.81802200
0.75902600
1.03685300
4.90843100
0.82037400
$-0.82252100$
4.04504700
$-0.94747300$
0.00924700
3.88339000
$-1.44822100$
$-0.94959400$
3.79422200
$-1.51085800$
0.91248600 
${ }^{3}$ B10-11TS-02

\begin{tabular}{|c|c|c|c|}
\hline 0 & -0.89895000 & 3.70202800 & 0.45546100 \\
\hline 0 & -1.65899000 & 1.25472500 & 1.40447900 \\
\hline 0 & 1.22380600 & 1.97406100 & 0.60405800 \\
\hline 0 & -0.96355600 & 1.57774800 & -1.20989000 \\
\hline Si & -2.55023400 & -0.15977000 & 1.13639100 \\
\hline Si & -1.78015000 & 0.21441100 & -1.78771900 \\
\hline 0 & -2.71717700 & -0.25074400 & -0.50465600 \\
\hline 0 & -4.03884900 & -0.04396700 & 1.80030400 \\
\hline $\mathrm{H}$ & -4.13423600 & -0.36514700 & 2.70615800 \\
\hline 0 & -2.68467100 & 0.57626600 & -3.09865200 \\
\hline $\mathrm{H}$ & -3.46771700 & 1.12280900 & -2.95462900 \\
\hline 0 & 0.82666200 & -0.64650400 & 1.48237100 \\
\hline 0 & 1.79326200 & -0.92041700 & -1.18745400 \\
\hline 0 & -0.14648200 & -2.53435000 & -0.15020100 \\
\hline 0 & -1.71816600 & -1.43570300 & 1.72835900 \\
\hline 0 & -0.69094500 & -0.91104100 & -2.21363500 \\
\hline Si & 0.49788900 & -1.86393000 & -1.52164500 \\
\hline 0 & 0.26466400 & -3.11141700 & 2.47640700 \\
\hline $\mathrm{H}$ & -0.15026600 & -3.97323400 & 2.34363500 \\
\hline 0 & 0.83996300 & -3.11206400 & -2.53561700 \\
\hline $\mathrm{H}$ & 1.23830400 & -2.86808600 & -3.38025700 \\
\hline V & 2.11959500 & 0.10942900 & 0.33320500 \\
\hline V & -0.58052900 & 2.17671700 & 0.38711400 \\
\hline Si & -0.15126700 & -1.93739500 & 1.40291500 \\
\hline C & 2.24312800 & 2.79931500 & -0.04940000 \\
\hline $\mathrm{H}$ & 2.83999400 & 3.25993700 & 0.75483400 \\
\hline $\mathrm{H}$ & 1.74613300 & 3.60998300 & -0.61307900 \\
\hline C & 3.03220500 & 1.82387300 & -0.87946800 \\
\hline $\mathrm{H}$ & 2.48167600 & 1.49408600 & -1.77537800 \\
\hline $\mathrm{H}$ & 4.00019000 & 2.23288600 & -1.18603000 \\
\hline C & 4.51457500 & 0.40666400 & 0.26746200 \\
\hline $\mathrm{H}$ & 4.91897400 & 1.34061400 & 0.66382600 \\
\hline $\mathrm{H}$ & 4.90486800 & 0.10160500 & -0.70638300 \\
\hline C & 3.98759800 & -0.54523400 & 1.14132300 \\
\hline $\mathrm{H}$ & 3.95875100 & -1.60367500 & 0.85358800 \\
\hline $\mathrm{H}$ & 3.92789200 & -0.33621800 & 2.21701800 \\
\hline
\end{tabular}

${ }^{3}$ B11-01

$\begin{array}{rrrr}0 & 1.17819700 & -3.46408400 & -1.55685400 \\ 0 & 0.92728000 & -1.97935200 & 0.78938900 \\ 0 & -0.73986000 & -1.56831300 & -1.51122000 \\ 0 & 2.03296900 & -0.80208800 & -1.46236400\end{array}$




\begin{tabular}{|c|c|c|c|}
\hline Si & 1.53076800 & -0.84810700 & 1.83728100 \\
\hline Si & 2.65745000 & 0.50564600 & -0.64927500 \\
\hline 0 & 2.54895100 & 0.12162000 & 0.96592100 \\
\hline 0 & 2.25581800 & -1.50056900 & 3.15031300 \\
\hline $\mathrm{H}$ & 2.96057200 & -2.14112900 & 2.99159900 \\
\hline 0 & 4.19935600 & 0.82705500 & -1.09450600 \\
\hline $\mathrm{H}$ & 4.76983100 & 0.06591600 & -1.25940800 \\
\hline 0 & -1.80212400 & 0.08446600 & 0.71816100 \\
\hline 0 & -0.81940200 & 1.63689200 & -1.68345900 \\
\hline 0 & -0.19381200 & 2.19519500 & 0.83109600 \\
\hline 0 & 0.28580200 & 0.06609700 & 2.43259800 \\
\hline 0 & 1.78767600 & 1.86606300 & -0.93355100 \\
\hline Si & 0.24953300 & 2.45488200 & -0.76694600 \\
\hline 0 & -1.87566700 & 1.58603800 & 2.91383500 \\
\hline $\mathrm{H}$ & -1.52191400 & 1.72728300 & 3.80162000 \\
\hline 0 & 0.25206800 & 4.05973600 & -1.08922800 \\
\hline $\mathrm{H}$ & 1.11085600 & 4.49933600 & -1.05370300 \\
\hline V & -1.68651900 & 0.04238100 & -1.34275700 \\
\hline V & 0.82641000 & -2.04033100 & -1.00511400 \\
\hline Si & -0.84976100 & 1.04168200 & 1.76508500 \\
\hline C & -2.56737900 & -1.03511600 & 1.27972600 \\
\hline $\mathrm{H}$ & -2.50203700 & -0.97041600 & 2.37577400 \\
\hline $\mathrm{H}$ & -2.06015900 & -1.95710700 & 0.95401100 \\
\hline C & -4.01589400 & -0.98219400 & 0.82501800 \\
\hline $\mathrm{H}$ & -4.54783700 & -1.76323800 & 1.39532900 \\
\hline $\mathrm{H}$ & -4.44191700 & -0.01357800 & 1.13951800 \\
\hline C & -4.24673900 & -1.20299400 & -0.67914700 \\
\hline $\mathrm{H}$ & -5.33414300 & -1.33256600 & -0.83724700 \\
\hline $\mathrm{H}$ & -3.79227600 & -2.16875800 & -0.97095800 \\
\hline C & -3.71532900 & -0.08219300 & -1.58580300 \\
\hline $\mathrm{H}$ & -3.97642600 & -0.26528800 & -2.64551100 \\
\hline $\mathrm{H}$ & -4.13986400 & 0.89988400 & -1.29586100 \\
\hline \multicolumn{4}{|c|}{${ }^{3} \mathrm{~B} 11-02$} \\
\hline 0 & -0.76231300 & -1.59253800 & -1.43658200 \\
\hline 0 & 1.98406600 & -1.14191500 & -0.37781900 \\
\hline 0 & -0.39402900 & -1.80148800 & 1.24816900 \\
\hline $\mathrm{Si}$ & -2.23713700 & -0.76733500 & -1.33467700 \\
\hline Si & -1.79203800 & -0.94826200 & 1.67709000 \\
\hline 0 & -2.64780300 & -0.85368800 & 0.26217900 \\
\hline 0 & -3.39524300 & -1.52375000 & -2.20298900 \\
\hline $\mathrm{H}$ & -3.49720000 & -1.24854900 & -3.12331200 \\
\hline 0 & -2.62914100 & -1.72332500 & 2.84385700 \\
\hline $\mathrm{H}$ & -3.08683100 & -2.53717100 & 2.59722200 \\
\hline
\end{tabular}




$\begin{array}{lrrr}\text { O } & 0.59335400 & 1.45384900 & -1.57307100 \\ \text { O } & 1.01659700 & 1.36247700 & 1.33786600 \\ \text { O } & -1.25133700 & 2.28776300 & 0.21483400 \\ \text { O } & -2.00182500 & 0.76647000 & -1.83876900 \\ \text { O } & -1.36876700 & 0.51846000 & 2.23526400 \\ \text { Si } & -0.52464100 & 1.82912700 & 1.63013200 \\ \text { O } & -1.22352900 & 3.30831400 & -2.30737000 \\ \text { H } & -2.04885600 & 3.77740700 & -2.13105600 \\ \text { O } & -0.67520400 & 3.08203300 & 2.67997100 \\ \text { H } & -0.40259000 & 2.91020700 & 3.58992600 \\ \text { V } & 1.79323300 & 0.89153000 & -0.27471900 \\ \text { Si } & -0.93581300 & 1.97937400 & -1.38881000 \\ \text { C } & 3.33533800 & -1.70646800 & -0.36531900 \\ \text { H } & 3.72341100 & -1.62684400 & -1.39313300 \\ \text { H } & 3.23786600 & -2.77107200 & -0.10948400 \\ \text { C } & 4.20315200 & -0.96598100 & 0.64342900 \\ \text { H } & 3.59641900 & -0.84134600 & 1.55892400 \\ \text { H } & 5.03597400 & -1.63328400 & 0.92262000 \\ \text { C } & 4.75820700 & 0.39399500 & 0.16272900 \\ \text { H } & 5.68162500 & 0.20718500 & -0.41787700 \\ \text { H } & 5.07567900 & 0.95877700 & 1.05445300 \\ \text { C } & 3.77677600 & 1.24288200 & -0.66117100 \\ \text { H } & 4.04970100 & 2.31322700 & -0.64881600 \\ \text { H } & 3.78558400 & 0.93031900 & -1.72962400 \\ \text { O } & 0.77998900 & -3.61134300 & -0.41437100 \\ \text { V } & 0.42697000 & -2.09921000 & -0.26395400\end{array}$

\section{${ }^{3}$ B12-01}

$\begin{array}{lrrr}\text { C } & -4.32482400 & 2.25005100 & 0.34485500 \\ \text { H } & -5.34452400 & 2.63185300 & 0.50274400 \\ \text { H } & -3.55398500 & 2.73865400 & 0.96114300 \\ \text { C } & -4.02521000 & 1.29112400 & -0.54880800 \\ \text { H } & -2.73672100 & 0.13052700 & 1.74672800 \\ \text { H } & -4.84331500 & 0.85156900 & -1.14297400 \\ \text { O } & 1.44275700 & 3.64721800 & -0.59676200 \\ \text { O } & 1.72486800 & 0.99689200 & -1.44324000 \\ \text { O } & -0.77432100 & 2.10129900 & -0.55020900 \\ \text { O } & 1.40044400 & 1.56601600 & 1.24574500 \\ \text { Si } & 2.15824600 & -0.58447500 & -1.21034700 \\ \text { Si } & 1.77595200 & 0.03489800 & 1.74884800 \\ O & 2.35286500 & -0.77946100 & 0.43026400 \\ 0 & 3.54631200 & -0.88475500 & -2.02231100 \\ \text { H } & 3.71758400 & -1.80431600 & -2.26172100 \\ 0 & 2.81198100 & 0.00474400 & 3.01289300\end{array}$


$\mathrm{H}$

0

0

0

0

0

Si

0

$\mathrm{H}$

0

$\mathrm{H}$

V

V

Si

${ }^{3}$ B12-02

C

$\mathrm{H}$

$\mathrm{H}$

C

$\mathrm{H}$

$\mathrm{H}$

0

0

0

Si

Si

0

0

$\mathrm{H}$

0

$\mathrm{H}$

0

0

0

0

0

Si

0

$\mathrm{H}$

0

$\mathrm{H}$

V

Si
3.63125400

$-1.55187600$

$-1.99712100$

$-0.66075200$

0.97391300

0.40353100

$-0.96510800$

$-1.04804000$

$-1.05275500$

$-1.82586600$

$-1.34938000$

$-2.06450000$

0.93299500

$-0.59286800$
0.50765900

$-0.69801300$

$-0.10540700$

$-2.22817400$

$-1.61607100$

$-0.72129400$

$-1.36920200$

$-3.38760900$

$-3.41630600$

$-2.14891500$

$-2.53362900$

0.77932400

2.18516100

$-1.97464600$
2.91928700

$-1.67791500$

1.16069500

0.33257600

$-1.70960800$

2.28843800

1.68062600

$-2.00730800$

$-2.97239300$

2.82512100

3.57252800

$-0.71942000$

$-0.37081300$

$-1.31436200$

\begin{tabular}{rrrr}
$C$ & 4.47084800 & 2.12874700 & 0.31844800 \\
$H$ & 5.47150000 & 2.58568100 & 0.34901100 \\
$H$ & 3.73625200 & 2.60218300 & 0.98991900 \\
$H$ & 4.16284500 & 1.05902500 & -0.43714100 \\
\hline & 1.87141900 & 2.84949300 & -0.59425200 \\
\hline & 4.95883200 & 0.63060200 & -1.06887500 \\
0 & -1.11240900 & 1.57285900 & 1.25496700 \\
Si & 1.30000200 & 2.08664500 & -0.39022900 \\
Si & -1.33821700 & 1.26580400 & -1.42767400 \\
0 & -1.87249700 & 0.11087000 & 1.66654500 \\
0 & -2.13278800 & -0.23167200 & -1.35663700 \\
$H$ & -2.52872800 & -0.40229400 & 0.23560600 \\
0 & -3.00398900 & 0.33669200 & 2.81787000 \\
$H$ & -3.80345200 & 0.82099000 & 2.57487300 \\
0 & -3.51018100 & -0.20204700 & -2.23045900 \\
0 & -3.44765500 & -0.46601600 & -3.15764900 \\
0 & 1.72482900 & -0.44504700 & 1.33723000 \\
0 & 1.51624500 & -0.77369600 & -1.56136000 \\
0 & 0.23559900 & -2.37517500 & 0.19735600 \\
Si & -0.75617700 & -0.92474700 & 2.23080900 \\
0 & -1.09827900 & -1.37829300 & -1.87704900 \\
$H$ & 0.40144800 & -1.95332900 & -1.40184100 \\
0 & 1.13449400 & -2.79763900 & 2.65792100 \\
$H$ & 1.35232900 & -2.51232100 & 3.55418700 \\
Si & 0.79140800 & -3.25431400 & -2.31992100 \\
\hline$i$ & 0.29212500 & -4.06393500 & -2.15390900 \\
& 2.27278000 & 0.29933300 & -0.26228500 \\
\hline & 0.63258000 & -1.62952200 & 1.62184900
\end{tabular}


0

V

3B13-01

C

$\mathrm{H}$

$\mathrm{H}$

C

$\mathrm{H}$

$\mathrm{H}$

0

0

0

0

Si

Si

0

0

$\mathrm{H}$

0

$\mathrm{H}$

0

0

0

0

0

Si

0

$\mathrm{H}$

0

H

V

V

Si

C

$\mathrm{H}$

$\mathrm{H}$

C

$\mathrm{H}$

$\mathrm{H}$

${ }^{3}$ B13-02

C

$\mathrm{H}$

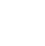

$-0.93382800$

$-0.53373900$

3.74722200

$-0.38109300$

2. 24980100

$-0.23996500$

$-4.01413100$

$-0.12007000$

$-2.36433500$

$-4.90547100$

0.09512300

$-2.97229600$

$-3.24673200$

$-0.72708900$

$-2.86749500$

$-3.87420900$

0.29029400

$-1.09144400$

$-1.63954800$

1.33006300

$-2.18295000$

$-4.68691800$

0.88309800

$-0.63868100$

0.52025700

$-3.68584000$

$-1.10706500$

1.05053700

$-1.86425300$

0.96472400

$-1.11994600$

$-1.56660400$

$-0.85597400$

1.60742800

$-1.19371500$

$-1.68567300$

1.91143500

$-0.60404200$

1.60962300

2.44070600

0.17731600

$-1.27758400$

2.68394800

0.16036400

0.35276900

2.96817800

$-1.20888900$

2.70288400

3.50912600

$-0.57185300$

3.18658200

3.89929200

0.29012900

$-2.00746200$

3.90775000

0.35952400

$-2.97047800$

$-1.60741900$

0.68282800

1.41648000

$-1.17454200$

1.24352400

$-1.33443600$

0.24435200

2.41884700

0.54386500

0.90835000

0.49553900

2. 31889700

1.49901000

1.48602700

$-1.67405600$

0.18167900

2.24225600

$-1.07667600$

$-0.56288800$

2.63920400

3.04535500

$-0.65386500$

2. 28873600

3.94012100

$-0.01130300$

3.64469900

$-1.89342000$

$-0.49032200$

4.35259200

$-1.44290300$

$-2.16914400$

$-0.22322700$

$-0.09402200$

0.47337700

$-2.17315500$

$-0.70202600$

$-0.29131600$

1.54095000

1.85627400

$-2.68085200$

$-2.17490400$

1.48626800

$-1.88961200$

$-1.89628400$

2.18858800

$-2.51027900$

$-3.06895400$

0.88031400

$-3.82702400$

$-1.47273100$

1.39658400

$-4.63607100$

$-1.78402400$

0.73014300

$-4.01522000$

$-0.60529800$

2.03769600

3.40083000

3.26591100

0.42174200

4.06183900

4.14416800

0.37162100 


\begin{tabular}{|c|c|c|c|}
\hline & 2.75856900 & 3.22530700 & 1.31515300 \\
\hline & 3.39295500 & 2.28725700 & -0.50332200 \\
\hline & 1.09282900 & 3.00082900 & -0.48574600 \\
\hline & 4.08013900 & 2.39875000 & -1.35970300 \\
\hline & -1.76138400 & 3.53818900 & -0.42561800 \\
\hline & -1.74141700 & 1.37482400 & 1.22995800 \\
\hline & 0.61594500 & 2.15659300 & -0.36305900 \\
\hline & -1.86818600 & 1.02250700 & -1.46917100 \\
\hline & -2.20006000 & -0.19956200 & 1.63046200 \\
\hline & -2.33222200 & -0.59921700 & -1.40322500 \\
\hline & -2.71502300 & -0.84495500 & 0.19164700 \\
\hline & -3.37744900 & -0.21350300 & 2.76175300 \\
\hline & -4.21262700 & 0.22027000 & 2.54744700 \\
\hline & -3.59603200 & -0.89802600 & -2.39328700 \\
\hline & -4.41353600 & -0.40852700 & -2.23910600 \\
\hline & 1.44312700 & 0.04820700 & 1.38725300 \\
\hline & 1.31051400 & -0.37231600 & -1.53443800 \\
\hline & 0.41470500 & -2.16450800 & 0.25595500 \\
\hline & -0.91773600 & -0.99864400 & 2.23759800 \\
\hline & -1.09264900 & -1.54074700 & -1.88152400 \\
\hline & 0.47117900 & -1.76318400 & -1.34770700 \\
\hline & 1.41776400 & -2.24620600 & 2.77864700 \\
\hline & 0.98551500 & -3.06569600 & 3.05110700 \\
\hline & 1.12646600 & -3.05265700 & -2.13454800 \\
\hline & 1.07843400 & -3.03108900 & -3.09864100 \\
\hline & 2.13638100 & 0.65484500 & -0.22871400 \\
\hline & -1.19220200 & 2.09772300 & -0.26531500 \\
\hline & 0.61830900 & -1.32298100 & 1.67735300 \\
\hline & 3.74181900 & -1.48559000 & 0.01864800 \\
\hline & 3.40513500 & -2.09633800 & -0.82390100 \\
\hline & 3.47206900 & -1.83019400 & 1.02103400 \\
\hline & 4.51968000 & -0.40294900 & -0.16611400 \\
\hline & 4.91937900 & 0.16918200 & 0.67622600 \\
\hline & 4.85438600 & -0.10435200 & -1.16422800 \\
\hline
\end{tabular}

${ }^{3}$ B13-14TS-01

$\begin{array}{lrrr}\mathrm{C} & -4.01145700 & -0.87267100 & -1.94235700 \\ \mathrm{H} & -4.29879500 & -0.65743700 & -2.97890300 \\ \mathrm{H} & -3.76812700 & -1.92301600 & -1.72437000 \\ \mathrm{C} & -3.93330000 & 0.08529000 & -0.99581300 \\ \mathrm{H} & -0.51153700 & -0.07924900 & -2.16927800 \\ \mathrm{H} & -4.24526100 & 1.12068600 & -1.18836000 \\ \mathrm{O} & 0.80596100 & -3.82024300 & -0.32236500 \\ \mathrm{O} & 1.16277300 & -1.60542900 & 1.35603100\end{array}$


0

0

Si

Si

0

0

$\mathrm{H}$

0

$\mathrm{H}$

0

0

0

0

0

Si

0

$\mathrm{H}$

0

$\mathrm{H}$

V

V

Si

C

$\mathrm{H}$

$\mathrm{H}$

C

$\mathrm{H}$

$\mathrm{H}$

${ }^{3}$ B13-14TS-02

C

$\mathrm{H}$

$\mathrm{H}$

C

$\mathrm{H}$

$\mathrm{H}$

0

0

0

Si

Si

0

0

$\mathrm{H}$

$$
\begin{array}{r}
-1.04725600 \\
1.59446300 \\
1.93760300 \\
2.54444100 \\
2.79643200 \\
2.91098700 \\
3.50073400 \\
3.94347700 \\
4.46982100 \\
-1.68283500 \\
-0.83200100 \\
0.09032800 \\
0.86548000 \\
1.72584500 \\
0.25136500 \\
-0.82170800 \\
-0.12219700 \\
-0.07382900 \\
-0.62028600 \\
-2.17467100 \\
0.58631300 \\
-0.41415000 \\
-3.37051300 \\
-2.77984500 \\
-3.48461000 \\
-4.33390000 \\
-5.27748500 \\
-4.40973200
\end{array}
$$

4.18233100

4.12998800

4.64404000

3.71042500

0.64700600

3.84326700

$-1.95402800$

0.38481300

$-1.81357500$

$-2.30995900$

$-2.18466100$

$-2.65721400$

$-3.56626800$

$-4.43933800$
$-1.87486100$

$-1.34656700$

$-0.19041500$

$-0.01037800$

0.24873800

$-0.35742200$

$-1.12210800$

$-0.21027500$

0.58127300

0.86675800

0.62986400

2. 50600800

0.99510100

1.30920900

1.94205800

3.04116900

3.33837700

3.09384300

3.83472500

$-0.39407300$

$-2.27212000$

1.83622900

$-1.50888600$

$-1.27730900$

$-2.56923100$

$-0.58096500$

$-0.93844000$

0.38553400

$-0.45257700$

$-1.38476900$

1.75059300

$-1.21918500$

0.39642500

3.05663000

3.05685000

$-2.04131700$

$-2.21149900$

1.18639500

$-1.58609400$

0.04025400

2.11685300

$-1.81755000$

$-1.48251300$

2.52886600

3.12422500

$-2.59627700$

$-2.30438200$

$-0.07217600$

$-0.20067500$

1.49280300

1.35929700

2. 25067700

1.11322700

0.90734100

0.49201100

1.41677300

$$
\begin{array}{rr}
-1.18836800 & -0.74014800 \\
-0.89411700 & -1.79611800 \\
-2.16269100 & -0.52942200 \\
-0.39152500 & 0.23686200 \\
3.36207900 & -0.12982600 \\
-0.70918300 & 1.28211900 \\
1.18568500 & 1.21237600 \\
2.42748800 & -0.12792100 \\
0.95564300 & -1.49518600 \\
-0.43897500 & 1.50184700 \\
-0.69062500 & -1.53922200 \\
-1.05147200 & 0.00190000 \\
-0.61113200 & 2.53079000 \\
-0.35321700 & 2.20865100
\end{array}
$$




\begin{tabular}{lrrr}
0 & -3.43298200 & -0.98769500 & -2.55083500 \\
\hline & -3.20194800 & -1.25810900 & -3.44890300 \\
0 & 1.23388300 & 0.21428900 & 1.53777500 \\
0 & 1.26720400 & 0.01260300 & -1.36013700 \\
0 & 0.58631800 & -2.03732300 & 0.22494500 \\
0 & -1.00940000 & -1.15915500 & 2.16905900 \\
Si & -0.84603500 & -1.51512000 & -1.98678400 \\
$O$ & 0.69523100 & -1.52359900 & -1.35188100 \\
$H$ & 1.40162500 & -2.15788100 & 2.82485300 \\
$O$ & 0.96647200 & -2.96400000 & 3.13022100 \\
$H$ & 1.52884900 & -2.60461800 & -2.26804700 \\
$V$ & 2.43645300 & -2.74788100 & -1.96917600 \\
$S i$ & 2.03337700 & 0.94445800 & 0.03872500 \\
$O$ & 0.58938900 & -1.27493200 & 1.70161600 \\
$V$ & -2.16817600 & 3.39729900 & -0.34818600 \\
$C$ & -1.39667600 & 2.05165900 & -0.20027300 \\
$H$ & 4.33672400 & 1.61938100 & 0.04781100 \\
$H$ & 4.87954200 & 1.41066300 & -0.87656700 \\
$C$ & 4.91388600 & 1.42971700 & 0.95734500 \\
$H$ & 3.36068600 & 2.63776000 & 0.04075200 \\
$H$ & 3.12522100 & 3.16706400 & 0.97398700 \\
& 3.17017600 & 3.19043700 & -0.88821400
\end{tabular}

3B14-01

\begin{tabular}{|c|c|c|c|}
\hline$C$ & 4.06357000 & -1.33360900 & 1.59831200 \\
\hline $\mathrm{H}$ & 4.25225300 & -1.02785000 & 2.63241100 \\
\hline $\mathrm{H}$ & 3.47210400 & -2.24462700 & 1.44761100 \\
\hline C & 4.57488900 & -0.65189400 & 0.56009500 \\
\hline $\mathrm{H}$ & 2.54795200 & 0.00401500 & 1.17109300 \\
\hline $\mathrm{H}$ & 5.16227200 & 0.25438700 & 0.76242100 \\
\hline 0 & -0.92716900 & -3.61733700 & -1.37362700 \\
\hline 0 & -1.97536800 & -1.02391200 & -1.43747700 \\
\hline 0 & 0.84332100 & -1.58019300 & -1.35376900 \\
\hline 0 & -0.88069900 & -2.01452300 & 0.90182400 \\
\hline Si & -2.73030000 & 0.26195500 & -0.71701600 \\
\hline Si & -1.55756900 & -0.85173000 & 1.86695000 \\
\hline 0 & -2.60599100 & 0.00996400 & 0.92234200 \\
\hline 0 & -4.29413200 & 0.30833800 & -1.19815800 \\
\hline $\mathrm{H}$ & -4.71892800 & 1.17530600 & -1.19193900 \\
\hline 0 & -2.27151200 & -1.45798500 & 3.20779100 \\
\hline $\mathrm{H}$ & -2.94837800 & -2.13377100 & 3.07387000 \\
\hline 0 & 0.62781200 & 1.56126500 & -1.71156100 \\
\hline 0 & 1.76206200 & 0.44199500 & 0.75703200 \\
\hline 0 & -0.12757500 & 2.27354900 & 0.76358800 \\
\hline
\end{tabular}




$\begin{array}{rrr}-1.98677900 & 1.68603100 & -1.08041400 \\ -0.37223700 & 0.16288800 & 2.42148600 \\ 0.65413900 & 1.25454200 & 1.76380900 \\ -0.57247500 & 3.98739300 & -1.19546200 \\ -0.72209200 & 4.22629000 & -2.11884600 \\ 1.56287600 & 1.96672100 & 2.91758200 \\ 1.16560600 & 2.08645100 & 3.79019400 \\ 1.66031600 & 0.09407000 & -1.26936300 \\ -0.70550900 & -2.14840900 & -0.87813700 \\ -0.49854400 & 2.38373200 & -0.86029400 \\ 3.67425600 & 0.09988500 & -1.70870900 \\ 4.09847900 & 1.10169400 & -1.49984100 \\ 3.79482700 & -0.08900700 & -2.79335800 \\ 4.39679500 & -0.99597700 & -0.89262100 \\ 3.86622700 & -1.96092100 & -0.98477300 \\ 5.40682400 & -1.16514300 & -1.31650900\end{array}$

${ }^{3}$ B14-02

\begin{tabular}{|c|c|c|c|}
\hline C & 3.89874900 & -1.06718800 & -0.22629700 \\
\hline $\mathrm{H}$ & 3.54655500 & -1.95536300 & 0.30536900 \\
\hline $\mathrm{H}$ & 3.87387100 & -1.10385700 & -1.31973200 \\
\hline C & 4.38094400 & 0.00045700 & 0.43638200 \\
\hline $\mathrm{H}$ & 0.83361700 & 3.28484000 & -0.14240400 \\
\hline $\mathrm{H}$ & 4.38981800 & -0.02653000 & 1.53426700 \\
\hline 0 & -1.82459200 & 1.17133400 & 1.34681800 \\
\hline 0 & 0.48675900 & 2.37895100 & -0.07734700 \\
\hline 0 & -1.88856300 & 1.12947800 & -1.36639700 \\
\hline Si & -2.27172400 & -0.44790500 & 1.54956400 \\
\hline Si & -2.30872400 & -0.50491000 & -1.50432100 \\
\hline 0 & -2.72536300 & -0.93812600 & 0.03982600 \\
\hline 0 & -3.55783000 & -0.57815300 & 2.54823100 \\
\hline $\mathrm{H}$ & -3.36953700 & -0.77931900 & 3.47377700 \\
\hline 0 & -3.54292800 & -0.72227200 & -2.54921800 \\
\hline $\mathrm{H}$ & -4.41352400 & -0.40000600 & -2.28314700 \\
\hline 0 & 1.28950900 & 0.01669300 & 1.49189600 \\
\hline 0 & 1.29954000 & -0.04738700 & -1.50759000 \\
\hline 0 & 0.46608200 & -2.09580200 & 0.02040600 \\
\hline 0 & -0.98154500 & -1.27244700 & 2.11776100 \\
\hline 0 & -1.02291300 & -1.33892500 & -2.04965000 \\
\hline Si & 0.55763700 & -1.49384200 & -1.52915600 \\
\hline 0 & 1.37021500 & -2.56094200 & 2.46713700 \\
\hline $\mathrm{H}$ & 1.63511100 & -2.25951000 & 3.34503400 \\
\hline 0 & 1.34256900 & -2.54188500 & -2.52078900 \\
\hline - & 0.90256000 & -3.38350300 & -2.69487 \\
\hline
\end{tabular}


1.94317800

0.88688300

$-0.02714200$

Si

0.58163900

$-1.45167700$

1.54003400

C

3.54578000

2.23091500

$-0.01637700$

$\mathrm{H}$

3.53076900

2.69137200

0.99319600

3.50038900

3.04714600

$-0.76126700$

C

4.76805900

1. 31353100

$-0.20215400$

4.96055100

1.15654100

$-1.27643400$

5.69761400

1.71225500

0.24463700

$-2.03191200$

3.49971900

$-0.04617200$

$-1.33034400$

2.10983100

$-0.03689100$

${ }^{3} \mathrm{C} 8$

0

0

0

Si

Si

0

0

$\mathrm{H}$

0

$\mathrm{H}$

0

0

0

0

0

Si

0

$\mathrm{H}$

0

$\mathrm{H}$

V

Si

C

$\mathrm{H}$

$\mathrm{H}$

C

$\mathrm{H}$

$\mathrm{H}$

Si

0
$-1.60152400$

0.51471200

$-1.62252200$

$-1.95052200$

$-1.94634500$

$-2.33617200$

$-3.19428800$

$-3.30163300$

$-3.18204300$

$-3.04832500$

1.71990000

1.76993300

0.82143100

$-0.64523300$

$-0.62360400$

0.90476700

1.60736200

1.51752500

1.53046600

2.28682300

1.85274700

0.89359000

3.97479000

3.63919200

4.39208400

3.96956600

4.38468600

3.63151100

$-1.12135200$

$-1.75632500$
1.27427400

2. 26561600

1.22473700

$-0.32657600$

$-0.39009800$

$-0.92474300$

$-0.44536500$

$-1.29774100$

$-0.60531200$

$-1.29190000$

$-0.04960800$

$-0.12693500$

$-2.15689100$

$-1.15737700$

$-1.20913500$

$-1.52887300$

$-2.56786800$

$-2.39858100$

$-2.63700000$

$-3.13930800$

1.05408700

$-1.47271100$

1.88291600

2.75293000

1.04624500

1.89116800

1.06213300

2.76900900

2.12769000

3.63759400
1.30338500

$-0.05727400$

$-1.34626400$

1.51318100

$-1.50515600$

0.01499400

2.57171000

3.01199300

$-2.55672100$

$-3.22220500$

1.44104300

$-1.42353900$

0.03848500

2.08308100

$-2.04240400$

$-1.49945000$

2.53989400

3.48598100

$-2.53716200$

$-2.20998500$

$-0.01951800$

1.54394500

$-0.70431700$

$-1.28240800$

$-1.27359100$

0.65386300

1.23607900

1.21862900

$-0.04281300$

$-0.06448700$ 
${ }^{3} \mathrm{C} 8$-9TS-01

$\begin{array}{lrrr}\text { O } & -1.33632900 & 1.71880600 & 0.71244700 \\ \text { O } & 0.10317100 & 1.91071600 & -1.59781300 \\ \text { O } & -2.03367200 & 0.28235000 & -1.39582200 \\ \text { Si } & -1.50525200 & 0.49860900 & 1.81587000 \\ \text { Si } & -2.17496200 & -1.15504400 & -0.59076000 \\ \text { O } & -2.15725100 & -0.79441200 & 1.02497900 \\ \text { O } & -2.48573900 & 0.89349400 & 3.06391900 \\ \text { H } & -2.12982700 & 1.50167300 & 3.72360900 \\ \text { O } & -3.57193400 & -1.93387400 & -0.93836300 \\ \text { H } & -3.54808800 & -2.54033800 & -1.68880200 \\ \text { O } & 1.93130400 & 0.78224200 & 0.63833100 \\ \text { O } & 1.40246400 & -0.86362500 & -1.71106700 \\ \text { O } & 1.08478100 & -1.76521800 & 0.79875300 \\ \text { O } & -0.00112700 & 0.10401500 & 2.38917100 \\ \text { O } & -0.89277100 & -2.12462200 & -0.96455700 \\ \text { Si } & 0.75862000 & -2.07075600 & -0.81206000 \\ \text { O } & 2.59047800 & -0.62675000 & 2.77969800 \\ \text { H } & 2.45531600 & -1.30176700 & 3.45667100 \\ \text { O } & 1.39552800 & -3.51433500 & -1.26478000 \\ \text { H } & 0.92435900 & -4.30114900 & -0.96361000 \\ \text { V } & 1.60405300 & 0.91064300 & -1.27823700 \\ \text { Si } & 1.39678900 & -0.40370300 & 1.67614700 \\ \text { C } & 3.63601800 & 1.69044100 & -1.07726800 \\ \text { H } & 3.61130900 & 2.75045300 & -1.36384000 \\ \text { H } & 4.25213300 & 1.05041300 & -1.72312400 \\ \text { C } & 3.63378400 & 1.39416200 & 0.31692500 \\ \text { H } & 4.16838500 & 0.51407700 & 0.68559400 \\ \text { H } & 3.56415200 & 2.20796700 & 1.04265300 \\ \text { Si } & -1.39575500 & 1.75590800 & -0.94946300 \\ \text { O } & -2.37625900 & 2.99277500 & -1.40127800 \\ \text { H } & & 3.42358000 & -2.23287900\end{array}$

${ }^{3} \mathrm{C} 8-9 T S-02$

$\begin{array}{lrrr}0 & 1.57774500 & 1.13845400 & -1.33846100 \\ 0 & -0.55371900 & 2.14947500 & -0.03123600 \\ 0 & 1.56525200 & 1.15007800 & 1.31249100 \\ \text { Si } & 1.82510000 & -0.50086200 & -1.51797000 \\ \text { Si } & 1.83777700 & -0.47832200 & 1.50870000 \\ \text { O } & 2.21785600 & -1.04718900 & -0.00134400 \\ \text { O } & 3.00340600 & -0.81935300 & -2.60773800 \\ \text { H } & 3.89693600 & -0.53407300 & -2.38031600\end{array}$




$\begin{array}{lrrr}\text { O } & 3.07996000 & -0.69331200 & 2.55234500 \\ H & 2.92864700 & -1.33769600 & 3.25547200 \\ \text { O } & -1.98857700 & -0.21200500 & -1.44233500 \\ \text { O } & -1.94707100 & -0.20234900 & 1.45543700 \\ \text { O } & -0.91617000 & -2.19517100 & 0.00375500 \\ \text { O } & 0.46512800 & -1.22411000 & -2.06371100 \\ \text { O } & 0.49440000 & -1.22800100 & 2.07398200 \\ \text { Si } & -1.03763300 & -1.57066000 & 1.53944600 \\ \text { O } & -1.75797900 & -2.65124800 & -2.55440000 \\ \text { H } & -1.16147300 & -3.30824100 & -2.93456000 \\ \text { O } & -1.61953000 & -2.68239800 & 2.59912800 \\ \text { H } & -2.54307200 & -2.93530500 & 2.47775800 \\ \text { V } & -2.04489800 & 0.91906500 & 0.00290900 \\ \text { Si } & -1.07184100 & -1.56798200 & -1.53007600 \\ \text { C } & -1.71374300 & 3.53324000 & -0.00344900 \\ H & -1.36694600 & 4.00540600 & -0.92669300 \\ \text { H } & -1.32981300 & 4.00405100 & 0.90568400 \\ \text { C } & -2.99275300 & 2.89196000 & 0.02145500 \\ \text { H } & -3.56361400 & 2.96565000 & 0.95697200 \\ \text { H } & -3.60048400 & 2.96713300 & -0.89036900 \\ \text { Si } & 1.09730200 & 2.01369700 & -0.01889400 \\ \text { O } & 1.66502100 & 3.54955800 & -0.01572100 \\ \text { H } & 2.61580700 & 3.68173700 & 0.08668700\end{array}$

\section{${ }^{3} \mathrm{C} 8-12 \mathrm{TS}-01$}

$\mathrm{C}$
$\mathrm{H}$
$\mathrm{H}$
$\mathrm{C}$
$\mathrm{H}$
$\mathrm{H}$
$\mathrm{O}$
$\mathrm{O}$
$\mathrm{O}$
$\mathrm{Si}$
$\mathrm{Si}$
$\mathrm{O}$
$\mathrm{O}$
$\mathrm{H}$
$\mathrm{O}$
$\mathrm{H}$
$\mathrm{O}$
$\mathrm{O}$
$\mathrm{O}$

$$
\begin{array}{r}
-4.19062600 \\
-5.14927100 \\
-3.45481200 \\
-3.91294500 \\
-3.04772500 \\
-4.69302400 \\
1.84694100 \\
-0.52870500 \\
1.11766300 \\
2.28488200 \\
1.52872800 \\
2.33023700 \\
3.74333500 \\
3.92987300 \\
2.41601900 \\
3.22167100 \\
-1.28070300 \\
-1.96666400 \\
-0.74034000
\end{array}
$$$$
\text { 2. } 33924700
$$$$
-0.06736000
$$$$
2.85244600
$$$$
-0.22834100
$$$$
2.89645900
$$$$
0.52948700
$$$$
1.11061400
$$$$
-0.54528800
$$$$
0.46699500
$$$$
0.45925100
$$$$
0.58605200
$$$$
-1.11813900
$$$$
0.80497400
$$$$
-1.41182900
$$$$
2.03835100
$$$$
-1.20063900
$$$$
1.74030800
$$$$
0.95951000
$$$$
-0.69311300
$$$$
-0.87999500
$$$$
0.39268400
$$$$
1.83013900
$$

$-0.60602600$

0.77780900

$-1.07698700$

$-1.51658100$

$-2.01890500$

$-1.61626900$

0.74963900

3.15673100

1.26244600

3.01460700

$-0.84357900$

$-1.73088200$

0.23770500

0.88643500

$-2.12089000$

0.57601700 
0

0

Si

0

$\mathrm{H}$

0

H

V

Si

Si

0

$\mathrm{H}$

${ }^{3} \mathrm{C} 8-12 \mathrm{TS}-02$

C

$\mathrm{H}$

$\mathrm{H}$

C

$\mathrm{H}$

$\mathrm{H}$

0

0

0

Si

Si

0

0

$\mathrm{H}$

0

$\mathrm{H}$

0

0

0

0

0

Si

0

$\mathrm{H}$

0

$\mathrm{H}$

V

Si

Si

0

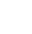

1.17368200

0.17387600

$-1.18703000$

$-0.87839100$

$-0.68865100$

$-2.20127700$

$-1.82509500$

$-1.81335100$

$-0.44347600$

1.02668300

1.74962200

1.45614000

$-1.83477800$

$-1.30967500$

$-0.36412700$

2. 37848500

$-0.98942200$

1.69311300

$-3.56290200$

$-1.64062800$

$-3.72262900$

$-2.57362200$

$-1.59400600$

2.82423600

$-2.18998800$

3.48453400

0.75390400

$-1.03953900$

$-2.09435600$

2.05065200

3.49779500

3.97412300

$-1.05286800$

$-0.66824600$

$-0.94239400$

$-1.72899900$

4.23889500

2.28885900

0.31272400

5.25198200

2.71594200

0.31992600

3.69927200

2. 35142000

1.26861300

3.67212500

1.72864700

$-0.77364900$

2. 22318900

2.12154500

$-0.72108800$

4.25894800

1.71117000

$-1.70579000$

$-0.90938700$

1.78051600

1.22657900

1.12358500

1.82572700

$-0.51195000$

$-1.36218900$

1.46154400

$-1.38844400$

$-1.67044200$

0.38155300

1.71347900

$-2.15015600$

0.00666400

$-1.25444700$

$-2.44777300$

$-0.18935000$

0.36430500

$-2.69402200$

0.65215300

2.95914300

$-3.37628300$

1.32222300

2.82607700

$-3.53443600$

0.06993900

$-2.12306100$

$-3.75141000$

$-0.72318600$

$-2.62938300$

1.94357600

$-0.67565300$

1.18305900

1.50231000

$-1.05562400$

$-1.60542300$

0.10963200

$-2.37551600$

0.24921000

$-0.56964700$

$-0.71070700$

2. 23661500

$-1.20412700$

$-1.21925600$

$-1.79636200$

0.18153000

$-2.01710000$

$-1.37244700$

1.14496900

$-2.71158500$

2.75242500

0.46283100

$-3.00514800$

3. 36937800

0.19497800

$-3.37835600$

$-2.28832300$

0.95520500

$-3.96168800$

$-2.17211900$

2.18071800

0.15561200

$-0.41809300$

0.67135200

$-1.62307500$

1.62158300

$-0.45644400$

2.28435700

$-0.27561600$

$-0.64496500$

3.90509700

$-0.37889300$ 
${ }^{3} \mathrm{C} 9-01$

0

0

0

Si

Si

0

0

$\mathrm{H}$

0

$\mathrm{H}$

0

0

0

0

0

Si

0

$\mathrm{H}$

0

H

V

Si

C

$\mathrm{H}$

$\mathrm{H}$

C

$\mathrm{H}$

$\mathrm{H}$

Si

0

$\mathrm{H}$

${ }^{3} \mathrm{C} 9-02$

0

0

0

Si

Si

0

0

$\mathrm{H}$

0

1.35831200

0.01065200

2.10395800

1.45785400

2.11298700

2.04254300

2.39388700

2.58474300

3.46966900

3.33043700

$-1.92712000$

$-1.47978600$

$-1.21487400$

$-0.06951000$

0.79575000

$-0.85060200$

$-2.65077900$

$-2.46976800$

$-1.38766400$

$-2.31753400$

$-1.55561500$

$-1.45134800$

$-3.18241700$

$-3.97923100$

$-2.99076300$

$-3.35536700$

$-3.34438500$

$-4.24088500$

1.47974900

2.50600400

2. 34395100

$-1.75617700$

$-1.71696900$

$-0.09256800$

$-0.64948100$

1.27692300

0.76156100

$-1.24652000$

$-0.66214300$

2.14911300

3.08331700

$-0.84922900$

1.04750900

1.65457100

$-0.33579200$

2. 21277000

2.14617900

0.33570400

$-0.02232700$

3.67697300

3.76416400

$-0.79811600$

0.23906200

$-1.66233900$

$-1.10429700$

$-2.60308000$

$-1.81676100$

$-2.86203000$

$-1.29666300$

$-1.61569000$

$-2.78088300$

$-3.10408100$

0.57147600

$-1.79792400$

$-1.35038800$

1.78195400

$-0.43161200$

1.14758400

2.98157700

3.72601300

$-0.70628400$

$-0.90627300$

0.48358000

$-1.68279100$

0.91763300

2. 36546000

$-0.74996200$

$-0.64526800$

2.80183100

3.68021900

$-0.89255900$

$-1.13582700$

$-1.52542900$

1.69378400

0.35346200

0.86626900

0.89000600

$-1.13870200$

$-1.48623400$

$-1.53884200$

$-1.08415300$

$-1.61375800$

$-2.50869100$

1.89834600

$-0.06305700$

$-1.32612000$

0.97982700

2.06813100

0.02432200

1.90629400

$-0.09043800$

1.32940500

1.09647600

$-1.51016200$

$-1.52343600$

1.08718700

$-1.53301100$

1.50824400

1.02970200

$-2.18467200$

$-0.01179000$

1.96367300

$-2.41823200$

$-2.57133500$

1.46663800

$-3.05533800$

$-3.09985200$ 


$\begin{array}{lrrr}\text { O } & 1.91610000 & -2.48897400 & 2.54318600 \\ \text { H } & 1.39678100 & -2.90092400 & 3.24542200 \\ \text { O } & -1.60771800 & 1.07570300 & -1.47951100 \\ \text { O } & -1.66831900 & 1.07089300 & 1.44382400 \\ \text { O } & -2.11542000 & -1.12709200 & -0.00810500 \\ \text { O } & -0.42247400 & -1.26749400 & -2.08715400 \\ \text { O } & -0.42579000 & -1.26350300 & 2.07253300 \\ \text { Si } & -1.82820500 & -0.56138500 & 1.53022400 \\ \text { O } & -3.08356300 & -1.01476900 & -2.47969800 \\ \text { H } & -3.10747700 & -0.63044400 & -3.36488900 \\ \text { O } & -2.98715700 & -1.06405900 & 2.57869300 \\ \text { H } & -3.87196500 & -0.70671000 & 2.43376800 \\ \text { V } & -1.03819000 & 2.02253700 & -0.00219900 \\ \text { Si } & -1.82301300 & -0.55119500 & -1.53248900 \\ \text { C } & 1.08549400 & 3.57789400 & 0.04324000 \\ \text { H } & 1.67542700 & 3.85343200 & -0.84225600 \\ \text { H } & 1.64426800 & 3.83266700 & 0.95476400 \\ \text { C } & -0.35419700 & 4.02378000 & 0.02406500 \\ \text { H } & -0.66088100 & 4.57587800 & 0.92661700 \\ \text { H } & -0.63171500 & 4.59227500 & -0.87780300 \\ \text { Si } & 2.15208300 & 0.86159700 & 0.01117200 \\ \text { O } & 3.56694500 & 1.67451100 & 0.01462400 \\ \text { H } & 4.39256400 & 1.17337600 & 0.01597700\end{array}$

\section{${ }^{3} \mathrm{C} 10-01$}

$\begin{array}{lrrr}0 & 1.54772500 & 0.52488700 & -1.81707600 \\ 0 & -0.92239600 & 1.59543100 & -1.27462900 \\ 0 & 1.18844200 & 2.07247400 & 0.30426400 \\ \text { Si } & 2.40469000 & -0.62967800 & -1.02365200 \\ \text { Si } & 1.87867900 & 1.06259900 & 1.40831700 \\ \text { O } & 2.65988700 & -0.11121000 & 0.52737600 \\ \text { O } & 3.79634900 & -0.91697100 & -1.83387200 \\ \text { H } & 4.39287400 & -1.57790800 & -1.46067200 \\ \text { O } & 2.92678200 & 1.88693400 & 2.35908600 \\ \text { H } & 2.84738800 & 1.71599700 & 3.30589600 \\ \text { O } & -1.10645300 & -1.32032800 & -1.00963300 \\ 0 & -1.79130200 & 0.04986800 & 1.40899900 \\ 0 & -0.02814500 & -1.97590000 & 1.29256400 \\ 0 & 1.50636200 & -2.02788100 & -0.94095500 \\ 0 & 0.73542200 & 0.35623100 & 2.36026600 \\ \text { Si } & -0.55357900 & -0.66588000 & 2.19577700 \\ 0 & -0.40491100 & -3.89446500 & -0.59767700 \\ \text { H } & 0.22527700 & -4.43391800 & -1.09162700 \\ O & -0.92597700 & -1.15318100 & 3.72088100\end{array}$


H

V

Si

C

$\mathrm{H}$

$\mathrm{H}$

C

$\mathrm{H}$

$\mathrm{H}$

C

$\mathrm{H}$

$\mathrm{H}$

C

$\mathrm{H}$

$\mathrm{H}$

Si

0

$\mathrm{H}$

${ }^{3} \mathrm{C} 10-02$

\section{0}

0

0

Si

Si

0

0

$\mathrm{H}$

0

$\mathrm{H}$

0

0

0

0

0

Si

0

$\mathrm{H}$

0

H

V

Si

C

$\mathrm{H}$
$-1.74913400$

$-2.09357200$

0.02533700

$-2.15035800$

$-2.36073200$

$-1.71690700$

$-3.28866300$

$-3.71551600$

$-4.09668300$

$-4.13104700$

$-4.69011700$

$-4.49786900$

$-3.10587900$

$-2.58397700$

$-2.77234200$

0.68710500

1.10889500

0.40891400
$-1.64806800$

0.46579300

$-2.34181500$

$-1.63282000$

$-2.71186000$

$-1.41001200$

$-0.71030600$

$-0.15934300$

$-1.21389800$

1.80016400

1.83921300

1.10691600

2.63967600

2.63301100

3.37012200

1.84312000

3.12271400

3.77056300
3.81423700

$-0.38513900$

$-0.29812000$

$-2.01404300$

$-1.94703500$

$-3.00087600$

$-1.64579300$

$-2.49893300$

$-1.08756800$

0.16688100

$-0.77273100$

0.93163100

0.41651700

1.37814900

$-0.32497400$

$-1.27194800$

$-2.21393000$

$-2.35920400$

$-1.80030000$

1.22580800

1.27898400

0.34092300

2.13286600

$-0.11128100$

$-1.79464700$

1.06387100

$-1.36681500$

$-2.15503400$

$-0.37279500$

1.55564700

$-2.18559800$

$-0.55349000$

$-1.46292900$

$-2.57015200$

$-1.00572900$

0.07948600

$-3.39549200$

$-0.43530300$

2.62177300

$-3.45055400$

$-1.23057700$

3.16633300

$-3.48067300$

$-0.78826400$

$-2.43673600$

$-3.28423300$

$-1.09299400$

$-3.33147200$

1.53659800

$-0.07983500$

1.47424100

1.49817700

$-0.19109900$

$-1.51099600$

0.55338700

$-2.15345400$

0.06874400

$-0.84807400$

$-1.16935000$

2.14514600

$-0.89483000$

$-1.39106600$

$-2.03259200$

0.67177100

$-1.59213300$

$-1.49443200$

1. 39709200

$-2.60791600$

2.53558800

1.53476400

$-2.34512300$

3.45409600

1.42116800

$-2.70174600$

$-2.45190700$

0.98214500

$-3.55826100$

$-2.52740100$

2.04790600

0.84062900

$-0.05845100$

0.68835300

$-1.47365100$

1.57198500

1.15410100

3.37806100

$-0.07666400$

0.91098800

3.90338400

0.85920300 


$\begin{array}{lrrr}\text { H } & 0.83381600 & 4.00025300 & -0.92610500 \\ \mathrm{C} & 2.57594400 & 2.87940300 & -0.14319500 \\ \mathrm{H} & 3.07362700 & 3.09326100 & -1.10532100 \\ \mathrm{H} & 3.21431800 & 3.23088100 & 0.68302200 \\ \mathrm{C} & 4.51335600 & 0.44106800 & 0.31277100 \\ \mathrm{H} & 4.49035000 & 0.59657700 & 1.39686400 \\ \mathrm{H} & 4.97443600 & 1.22987200 & -0.28832500 \\ \mathrm{C} & 4.09475400 & -0.71505600 & -0.23888300 \\ \mathrm{H} & 4.16511200 & -0.89937400 & -1.31411100 \\ \mathrm{H} & 3.70550200 & -1.53311200 & 0.37415400 \\ \mathrm{Si} & -1.32433400 & 2.00119100 & -0.09718600 \\ \mathrm{O} & -1.86288200 & 3.54458600 & -0.18164600 \\ \mathrm{H} & -2.81561900 & 3.69522100 & -0.13985300\end{array}$

${ }^{3}$ C10-11TS-01

$\begin{array}{lrrr}\text { O } & -0.89032700 & 1.67015700 & 1.26620400 \\ \text { O } & 1.19398600 & 1.83884300 & -0.45169500 \\ \text { O } & -1.38462500 & 1.54183800 & -1.32934100 \\ \text { Si } & -1.88208100 & 0.42141000 & 1.68925600 \\ \text { Si } & -2.38184600 & 0.22585000 & -1.27000500 \\ \text { O } & -2.75221500 & 0.01730000 & 0.34248200 \\ \text { O } & -2.83284400 & 0.76725900 & 2.97608600 \\ \text { H } & -3.40983400 & 1.53700400 & 2.89555600 \\ \text { O } & -3.72891300 & 0.43821600 & -2.17785000 \\ \text { H } & -4.15687000 & 1.30083600 & -2.11159200 \\ \text { O } & 1.53184900 & -0.82836600 & 1.11876700 \\ \text { O } & 1.07565900 & -0.93782100 & -1.70127900 \\ \text { O } & -0.26960000 & -2.42837000 & 0.05453800 \\ \text { O } & -0.95857300 & -0.86535200 & 2.16513000 \\ \text { O } & -1.64158400 & -1.12382500 & -1.83124700 \\ \text { Si } & -0.20637100 & -1.90998700 & -1.54733500 \\ \text { O } & 0.72037600 & -2.92254900 & 2.56641600 \\ \text { H } & 0.07232700 & -3.26633300 & 3.19487600 \\ \text { O } & -0.13341100 & -3.21919800 & -2.53586200 \\ \text { H } & -0.98428800 & -3.54695900 & -2.85285700 \\ \text { V } & 2.10312500 & 0.20106000 & -0.61772100 \\ \text { Si } & 0.19689900 & -1.81315900 & 1.48482600 \\ \text { C } & 2.14885800 & 0.13809200 & 2.02756300 \\ \text { H } & 2.33834400 & -0.35695500 & 2.99738600 \\ \text { H } & 1.44381100 & 0.96913200 & 2.17870600 \\ \mathrm{C} & 3.41966000 & 0.56718900 & 1.32300900 \\ \text { H } & 3.81507100 & 1.47054400 & 1.80305800 \\ \text { H } & 4.16678500 & -0.23934400 & 1.39073200 \\ \mathrm{C} & 4.19902100 & 1.41441800 & -0.53138200\end{array}$




$\begin{array}{lrrr}\text { H } & 5.15063200 & 1.16069800 & -0.06173300 \\ \text { H } & 3.85916000 & 2.43917700 & -0.35814100 \\ \text { C } & 3.81652300 & 0.77232400 & -1.72684400 \\ \text { H } & 3.27803100 & 1.35130200 & -2.48998300 \\ \text { H } & 4.39596100 & -0.08287300 & -2.09606000 \\ \text { Si } & -0.35833100 & 2.24216900 & -0.21783800 \\ \text { O } & -0.58126600 & 3.87449200 & -0.25067200 \\ \text { H } & 0.21076100 & 4.37830300 & -0.47453200\end{array}$

${ }^{3}$ C10-11TS-02

$\begin{array}{lrrr}\text { O } & 1.49101200 & 1.13208100 & -1.50639500 \\ \text { O } & -0.98143100 & 1.93524700 & -0.65655000 \\ \text { O } & 1.10075600 & 1.81109500 & 1.04040100 \\ \text { Si } & 2.42532800 & -0.22296200 & -1.19878600 \\ \text { Si } & 1.82625200 & 0.44685700 & 1.68736100 \\ \text { O } & 2.70977400 & -0.18365800 & 0.43207200 \\ \text { O } & 3.84620100 & -0.10953200 & -2.00479600 \\ \text { H } & 3.97618700 & -0.75110600 & -2.71460500 \\ \text { O } & 2.78020800 & 0.83431500 & 2.95917900 \\ \text { H } & 3.60604600 & 1.29184400 & 2.75885300 \\ \text { O } & -0.95522200 & -0.86527900 & -1.47799500 \\ \text { O } & -1.79800700 & -0.73859200 & 1.23321900 \\ \text { O } & 0.11560600 & -2.47890700 & 0.38878700 \\ \text { O } & 1.62294600 & -1.57829600 & -1.63375800 \\ \text { O } & 0.69112000 & -0.59043000 & 2.21445400 \\ \text { Si } & -0.49929900 & -1.63089700 & 1.67194300 \\ \text { O } & -0.29092800 & -3.41958500 & -2.12536100 \\ \text { H } & 0.11987400 & -4.24334900 & -1.83397500 \\ \text { O } & -0.82407600 & -2.73319000 & 2.84965600 \\ \text { H } & -1.16880300 & -2.36998800 & 3.67472300 \\ \text { V } & -2.08625800 & 0.16736300 & -0.37408800 \\ \text { Si } & -3.07627700 & -2.08824000 & -1.23023600 \\ \text { C } & -1.89687700 & 2.86237900 & 0.04670700 \\ \text { H } & -2.38548100 & 3.46211400 & -0.73643200 \\ \text { H } & -1.29868200 & 3.54449200 & 0.67573700 \\ \text { C } & -2.85459600 & 1.98526400 & 0.79949700 \\ \text { H } & -2.42745700 & 1.59217100 & 1.73437800 \\ \text { H } & -3.78540800 & 2.51518900 & 1.02511500 \\ \text { C } & -4.45244900 & 0.69555500 & -0.37454100 \\ \text { H } & -4.73712000 & 1.64431400 & -0.83468000 \\ \text { H } & -4.89444900 & 0.49684600 & 0.60446600 \\ \text { H } & -4.01086400 & -0.35722700 & -1.17178600 \\ \text { H } & -4.08505400 & -1.39231400 & -0.81541500 \\ \text { H } & -397000 & -0.22682100 & -2.25541100\end{array}$


Si

0

$\mathrm{H}$
0.69083600

0.89136700

1.78586400
2.15996800

3.74228800

4.10481500
$-0.51814300$

$-0.88650300$

$-0.85920100$

${ }^{3} \mathrm{C} 11-01$

0

0

0

Si

Si

0

0

$\mathrm{H}$

0

$\mathrm{H}$

0

0

0

0

0

Si

0

$\mathrm{H}$

0

$\mathrm{H}$

V

Si

C

$\mathrm{H}$

$\mathrm{H}$

C

$\mathrm{H}$

$\mathrm{H}$

C

$\mathrm{H}$

$\mathrm{H}$

C

$\mathrm{H}$

$\mathrm{H}$

Si

0

$\mathrm{H}$
0.90861500
-0.60861000
2.02754200
1.50730900
2.67526700

2. 58015500

2.17324100

2.95329700

4.21812400

4.77238900

$-1.83332100$

$-0.77846000$

$-0.13528500$

0.27297100

1.83464500

0.30804500

$-1.80831400$

$-1.43011700$

0.37195300

1.24644100

$-1.63034000$

$-0.83009800$

$-2.62650000$

$-2.59573700$

$-2.11913600$

$-4.06044000$

$-4.62245800$

$-4.47643200$

$-4.25231000$

$-5.33819300$

$-3.81187300$

$-3.65508800$

$-3.87110900$

$-4.08843300$

0.84661300

1.19082800

2.03261800
$-2.08894800$

$-1.38504300$

$-0.76426000$

$-1.13112400$

0.44890500

$-0.08773200$

$-1.98376200$

$-2.51487000$

0.78112100

0.02924100

0.02957200

1.77902200

2.04848500

$-0.27496300$

1.84694300

2.45941900

1.26693400

1.27215700

4.09049400

4.48658400

0.13219600

0.81989500

$-1.11971700$

$-1.13487800$

$-2.02216900$

$-1.01894000$

$-1.81278900$

$-0.05653800$

$-1.16967900$

$-1.26440900$

$-2.13391600$

$-0.03036600$

$-0.17188600$

0.94636300

$-1.90473600$

$-3.33014500$

$-3.74595100$
0.46154100

$-1.68463800$

$-1.53263600$

1.66599600

$-0.61540300$

0.96029800

2.89395600

2.69260100

$-1.05282300$

$-1.29433500$

0.69775200

$-1.53345600$

1.02978300

2.35646300

$-0.75660500$

$-0.53932800$

3.05984500

3.94863900

$-0.68559000$

$-0.58407600$

$-1.36329900$

1.82885100

1.14944700

2.24888900

0.77274200

0.65860000

1.18087700

1.00500700

$-0.85906000$

$-1.05233100$

$-1.17581900$

$-1.69656600$

$-2.77290800$

$-1.39646500$

$-1.20720100$

$-1.94707500$

$-1.72678200$

${ }^{3} \mathrm{C} 11-02$ 
0

0

0

Si

Si

0

0

$\mathrm{H}$

0

$\mathrm{H}$

0

0

0

0

0

Si

0

$\mathrm{H}$

0

H

V

Si

C

$\mathrm{H}$

$\mathrm{H}$

C

$\mathrm{H}$

$\mathrm{H}$

C

$\mathrm{H}$

$\mathrm{H}$

C

$\mathrm{H}$

$\mathrm{H}$

Si

0

$\mathrm{H}$

${ }^{3} \mathrm{C} 12-01$
$-0.30016700$

1.85794500

$-0.52556300$

$-1.68537700$

$-2.07378900$

$-2.54856400$

$-2.51764100$

$-2.70779300$

$-3.09014100$

$-3.42819900$

1.04307900

0.52233600

$-1.29322800$

$-1.31304600$

$-2.01094600$

$-1.04575100$

$-0.50270200$

$-1.34673700$

$-1.52641100$

$-2.47039900$

1.72891500

$-0.47722900$

2.99864000

2.82915100

2.96528400

4. 32185000

4.37276800

5.10271300

3.71801300

3.85601800

3.96750800

4.60840500

4.49879700

5.67664600

0.45140100

0.97691700

0.49325300
$-2.09516400$

$-1.12702000$

$-1.34026400$

$-1.30266100$

$-0.69813700$

$-1.04621300$

$-2.28241600$

$-1.92116700$

$-1.35682900$

$-2.24197100$

1.11204200

1.81933300

2.15467100

0.10747000

0.90342000

2.13346400

2.52937900

2.96272400

3. 54362500

3. 62509100

0.94987900

1.50035700

$-1.63778300$

$-1.33933700$

$-2.73397700$

$-1.11098900$

$-1.32298200$

$-1.72815200$

1.34432400

1.20201300

2. 39684100

0.37470200

0.56238200

0.55843600

$-2.07720000$

$-3.56465300$

$-4.32924200$

$-0.99366800$

0.38945000

1.55641000

$-1.50004300$

1.44266000

$-0.10798800$

$-2.51194700$

$-3.38694800$

2. 54054100

2.35785600

$-1.39093300$

1.44029500

$-0.52000900$

$-2.23433500$

1.72239700

1.12784000

$-3.09541300$

$-3.27274300$

1.81649500

2.00014300

0.33861600

$-1.81400700$

$-0.39410400$

$-1.44185200$

$-0.32458300$

0.13592200

1.21846800

$-0.34157500$

0.64977000

1.74343200

0.41974100

$-0.13568800$

$-1.22048200$

0.08887400

0.46891600

0.90319800

0.56504500

C

$-4.23640600$

2.23785400

0.24907100

$-5.24462100$

2.65782000

0.38446800

$-3.48243500$

2.63085600

0.94976000

$-3.92392400$

1. 34888400

$-0.71086000$

$-2.77793300$

0.22942600

1.58828200 
$\mathrm{H}$

0

0

0

Si

Si

0

0

$\mathrm{H}$

0

$\mathrm{H}$

0

0

0

0

0

Si

0

$\mathrm{H}$

0

$\mathrm{H}$

V

Si

0

$\mathrm{H}$

Si
$-4.73187000$

1.72076100

$-0.68048100$

1.19537200

2. 21957200

1.68592100

2. 38599500

3.63758400

3.79157700

2.65803700

3.45580900

$-1.44310200$

$-1.98831500$

$-0.60050100$

1.10237800

0.36240400

$-0.97070300$

$-0.87458600$

$-0.98172900$

$-1.86405400$

$-1.41028500$

$-1.96380900$

$-0.47973000$

1.55378900

1.04325500

0.91248200
1.01440200

0.99571500

2.08885700

1.70792800

$-0.53658200$

0.29505300

$-0.61119700$

$-0.82896600$

$-1.74181600$

0.51677700

1.04322500

$-0.79263100$

$-0.01454600$

$-2.17361300$

$-1.65843800$

$-0.51364700$

$-1.22813300$

$-3.48421200$

$-3.52735800$

$-1.94380600$

$-2.25438300$

0.77054800

$-2.01745600$

3.63136700

4. 21387300

2.13956400

$-1.38329300$

$-1.43122200$

$-0.87652200$

1.06105500

$-1.09193600$

1.75529100

0.56333900

$-1.85484700$

$-2.12857700$

3.05030300

2.91221900

$-1.69622500$

1.07499100

0.45157800

$-1.54987500$

2. 33964800

1.72254300

$-1.82717100$

$-2.78566300$

2.88502300

3.67945300

$-0.85453900$

$-1.20797700$

$-0.76332500$

$-1.33937000$

$-0.52550300$

${ }^{3} \mathrm{C} 12-02$

C

$\mathrm{H}$

$\mathrm{H}$

C

$\mathrm{H}$

$\mathrm{H}$

0

0

0

Si

Si

0

0

$\mathrm{H}$

0

$\mathrm{H}$
4.26862300

5.22664800

3.53551200

4.01205200

1.61797500

4.80911200

$-1.01246300$

1.11098400

$-1.29233800$

$-1.79706200$

$-2.11036700$

$-2.51791700$

$-2.89312200$

$-3.72266600$

$-3.46167800$

$-3.49201200$
2.36747000

2.90883200

2.77146900

1.27844000

2.86931800

0.92792400

1.63936100

2.08996200

1.38268900

0.21545200

$-0.07859500$

$-0.28314500$

0.46239500

0.89015200

0.02144300

$-0.55360700$
0.23447200

0.21321400

0.95088800

$-0.51312400$

$-0.61244900$

$-1.19063400$

1.24560500

$-0.32427100$

$-1.38158800$

1.66184400

$-1.33749800$

0.25344600

2.84782400

2.60228700

$-2.25266600$

$-3.02786000$ 


$\begin{array}{lrrr}0 & 1.80016300 & -0.52148600 & 1.31616600 \\ 0 & 1.48993700 & -0.74965300 & -1.57680400 \\ 0 & 0.20356000 & -2.36230700 & 0.16564300 \\ 0 & -0.71024400 & -0.86794200 & 2.20211500 \\ \text { O } & -1.13879400 & -1.27869100 & -1.86902100 \\ \text { Si } & 0.34819200 & -1.90552200 & -1.42650000 \\ \text { O } & 1.08055500 & -2.84113900 & 2.62325700 \\ \text { H } & 1.32499100 & -2.56658900 & 3.51593200 \\ \text { O } & 0.68880900 & -3.19978700 & -2.37587700 \\ \text { H } & 0.23006800 & -4.02025400 & -2.15559700 \\ \text { V } & 2.20948500 & 0.33475800 & -0.26696800 \\ \text { Si } & 0.63800100 & -1.64174900 & 1.59394900 \\ \text { O } & -0.77421100 & 3.87967800 & -0.36354200 \\ \text { H } & -1.63757400 & 4.22390700 & -0.62625000 \\ \text { Si } & -0.57734800 & 2.26776800 & -0.20177000\end{array}$

\section{${ }^{3} \mathrm{C} 13-01$}

\begin{tabular}{|c|c|c|c|}
\hline C & 3.91540800 & 0.11909800 & -2.50431600 \\
\hline $\mathrm{H}$ & 4.73374400 & -0.18964100 & -3.17197900 \\
\hline $\mathrm{H}$ & 3.27379500 & 0.92207000 & -2.89491100 \\
\hline C & 3.71218700 & -0.42024700 & -1.28933100 \\
\hline $\mathrm{H}$ & 1.15758100 & -1.04032500 & -2.27622600 \\
\hline $\mathrm{H}$ & 4.39935000 & -1.21733500 & -0.95480000 \\
\hline 0 & -0.88183000 & 1.93439900 & 0.85184200 \\
\hline 0 & 1.16959700 & 1.62875100 & -0.85297400 \\
\hline 0 & -1.41347100 & 1.22499900 & -1.64534000 \\
\hline $\mathrm{Si}$ & -1.83271500 & 0.80225900 & 1.57665000 \\
\hline Si & -2.45321300 & 0.01289500 & -1.25993300 \\
\hline 0 & -2.72934700 & 0.09310400 & 0.36952200 \\
\hline 0 & -2.77398800 & 1.53746200 & 2.69669800 \\
\hline $\mathrm{H}$ & -3.12184500 & 0.97297800 & 3.39822900 \\
\hline 0 & -3.81806600 & 0.15031400 & -2.14786800 \\
\hline $\mathrm{H}$ & -4.31612800 & -0.66129900 & -2.30694100 \\
\hline 0 & 1.58184000 & -0.68849200 & 1.39335100 \\
\hline 0 & 0.92107300 & -1.17021700 & -1.34335400 \\
\hline 0 & -0.36572100 & -2.37204000 & 0.59523000 \\
\hline 0 & -0.92563200 & -0.36937400 & 2.30055000 \\
\hline 0 & -1.74754900 & -1.45913700 & -1.57730800 \\
\hline Si & -0.39740600 & -2.20080200 & -1.02524400 \\
\hline 0 & 0.46898200 & -2.54982500 & 3.08501800 \\
\hline $\mathrm{H}$ & 0.79941000 & -2.18664400 & 3.91593100 \\
\hline 0 & -0.25062500 & -3.61001700 & -1.83991500 \\
\hline $\mathrm{H}$ & 0.32129700 & -4.28247800 & -1.44727000 \\
\hline$V$ & 2.13139600 & 0.19831300 & -0.150133 \\
\hline
\end{tabular}




\section{${ }^{3} \mathrm{C} 13-02$}

C

$\mathrm{H}$

$\mathrm{H}$

C

$\mathrm{H}$

$\mathrm{H}$

0

0

0

Si

Si

0

0

$\mathrm{H}$

0

$\mathrm{H}$

0

0

0

0

0

Si

0

$\mathrm{H}$

0

H

V

Si

C

$\mathrm{H}$

$\mathrm{H}$

C
3.19417700

3.79022700

2.59089900

3.22090000

0.86230700

3.86882700

$-1.68474400$

0.43857400

$-1.80363100$

$-2.18379400$

$-2.33535200$

$-2.74367900$

$-3.33976500$

$-4.10536600$

$-3.60895000$

$-4.46239200$

1.46812300

1.29339800

0.38478400

$-0.93406400$

$-1.13823600$

0.42552800

1.35153000

0.87780700

1.04184500

0.89988500

2.07364800

0.59332100

3.77854000

3.46739100

3.52167700

4.50531000
3.36098400

4.28386500

3.26931500

2. 38974200

2.97471000

2. 55486900

1.51081400

2.09789800

1.03166400

$-0.00595500$

$-0.54962200$

$-0.73181400$

0.08882100

0.64572600

$-0.83523100$

$-0.47331900$

0.08890600

$-0.43700900$

$-2.13921500$

$-0.84027200$

$-1.56596300$

$-1.80024900$

$-2.13579700$

$-2.91573500$

$-3.13147200$

$-3.18073300$

0.68259400

$-1.23984300$

$-1.34714200$

$-1.97783900$

$-1.69165400$

$-0.23080200$
0.36169900

0.29647400

1.27830000

$-0.57085200$

$-0.45697000$

$-1.44921700$

1.12741100

$-0.44503500$

$-1.49683700$

1.61550000

$-1.36982000$

0.22807500

2.76805900

2.58154100

$-2.35618300$

$-2.08841500$

1. 37848300

$-1.53243000$

0.33415500

2. 24930900

$-1.81233800$

$-1.28502200$

2.87215000

3.18781200

$-2.03442100$

$-2.98797800$

$-0.27283100$

1.71974700

$-0.01479100$

$-0.85200100$

0.99065200

$-0.20827400$ 
$\mathrm{H}$

$\mathrm{H}$

0

$\mathrm{H}$

Si

${ }^{3} \mathrm{C} 13-14 \mathrm{TS}-01$

$\mathrm{H}$

$\mathrm{H}$

C

$\mathrm{H}$

$\mathrm{H}$

0

0

0

Si

Si

0

0

$\mathrm{H}$

0

$\mathrm{H}$

0

0

0

0

0

Si

0

$\mathrm{H}$

0

$\mathrm{H}$

V

Si

C

$\mathrm{H}$

$\mathrm{H}$

C

$\mathrm{H}$

$\mathrm{H}$

Si

0

$\mathrm{H}$
4.87600700

4.82788200

$-1.78659000$

$-1.98458100$

$-1.24447300$

4.00024100

4.29013800

3.78523400

3.88496300

0.56844800

4.16466700

$-1.02358100$

0.99321800

$-1.55544500$

$-1.90461100$

$-2.54902900$

$-2.83290700$

$-2.82441700$

$-3.40784500$

$-3.92198800$

$-4.39835100$

1.62268700

0.83416200

$-0.16568700$

$-0.92666200$

$-1.77136000$

$-0.32308600$

0.76450000

0.05005700

$-0.10511000$

0.46022000

2.13993400

0.35679800

3.37355200

2.79453500

3.51195400

4. 31399300

5.26134800

4.37845700

$-0.55699900$

$-0.87933600$

$-0.10148100$
0.36704300

0.07472400

3.60182400

3.88244500

2.06888400

0.62939900

$-1.20835200$

$-0.55581500$

$-1.45872900$

$-0.35117500$

0.76639800

$-2.02358700$

0.47879700

$-3.04186400$

1.83439300

$-1.87329400$

$-0.12529400$

$-1.01796300$

$-0.04228300$

$-2.22067800$

$-1.17951000$

$-1.14955100$

1.69112300

1.18379400

1.84953100

$-0.61422400$

1.39340300

$-1.39546500$

0.40102000

1.71853100

0.09747800

$-1.19719000$

$-0.07960600$

0.42257100

0.76606100

3.02314100

1.53025100

2.93494000

0.31462100

$-2.05750500$

$-0.48229400$

$-2.32264900$

$-0.74464100$

1.23276500

$-0.73147000$

$-1.58924100$

$-2.43397300$

0.16727600

$-0.83421300$

2.17472600

$-1.26855800$

$-1.73606400$

$-1.95763400$

$-1.38592500$

$-2.85887300$

2.67012500

$-3.16171800$

3.24444800

$-3.19978000$

$-2.42875500$

$-3.92094400$

$-2.12390700$

0.44303100

$-0.09455100$

$-1.69808500$

1.58249000

1.60516800

1.26160600

1.42873200

2.17309200

2.65058000

0.96752400

0.63674500

0.84226700

0.95457900

0.40422100

$-0.30375800$

1.40003700

2. 21724400

$-0.33181700$

3.82285500

$-0.49481400$

4.39322900

$-0.46348200$ 
${ }^{3} \mathrm{C} 13-14 T S-02$

\begin{tabular}{|c|c|c|c|}
\hline C & 4.16219800 & -1.08923000 & -0.71294800 \\
\hline $\mathrm{H}$ & 4.06768200 & -0.82922400 & -1.77480300 \\
\hline $\mathrm{H}$ & 4.66375700 & -2.04030700 & -0.48755000 \\
\hline C & 3.68922300 & -0.28039700 & 0.25346300 \\
\hline $\mathrm{H}$ & 0.38511800 & 3.31364000 & 0.02435700 \\
\hline $\mathrm{H}$ & 3.85718000 & -0.56149800 & 1.30402900 \\
\hline 0 & -1.93569500 & 1.27628700 & 1.15557900 \\
\hline 0 & 0.19586100 & 2.38212900 & -0.16242800 \\
\hline 0 & -1.76276300 & 1.04252100 & -1.47948200 \\
\hline Si & -2.33499500 & -0.31504200 & 1.45431400 \\
\hline Si & -2.17563600 & -0.57115500 & -1.55708200 \\
\hline 0 & -2.68098900 & -0.95998700 & -0.02835400 \\
\hline 0 & -3.65107600 & -0.39614200 & 2.42449500 \\
\hline $\mathrm{H}$ & -3.50265100 & -0.77159900 & 3.30129800 \\
\hline 0 & -3.38593600 & -0.78219100 & -2.63768700 \\
\hline $\mathrm{H}$ & -3.19760200 & -1.39545400 & -3.35933400 \\
\hline 0 & 1.20755100 & 0.23073700 & 1.56072300 \\
\hline 0 & 1.25455700 & 0.00850700 & -1.35683100 \\
\hline 0 & 0.53546500 & -2.01896800 & 0.24155300 \\
\hline 0 & -1.07038500 & -1.08641000 & 2.15999300 \\
\hline 0 & -0.87820700 & -1.47883400 & -1.98468800 \\
\hline Si & 0.65586400 & -1.51561200 & -1.34032800 \\
\hline 0 & 1.31358900 & -2.14369100 & 2.84733400 \\
\hline $\mathrm{H}$ & 0.95854100 & -3.02786500 & 3.00369100 \\
\hline 0 & 1.48658400 & -2.61662600 & -2.23731300 \\
\hline $\mathrm{H}$ & 2.39948800 & -2.73979700 & -1.94568900 \\
\hline V & 1.95898200 & 0.98007900 & 0.04352400 \\
\hline Si & 0.53206400 & -1.24438500 & 1.71194100 \\
\hline C & 4.22123700 & 1.74977000 & 0.00461100 \\
\hline $\mathrm{H}$ & 4.74399300 & 1.54976900 & -0.93356800 \\
\hline $\mathrm{H}$ & 4.83626300 & 1.60170200 & 0.89684700 \\
\hline C & 3.20351500 & 2.72857100 & 0.01355200 \\
\hline $\mathrm{H}$ & 2.97574100 & 3.26032500 & 0.94751800 \\
\hline $\mathrm{H}$ & 2.96158700 & 3.26091500 & -0.91515200 \\
\hline Si & -1.45492500 & 2.04561400 & -0.21745300 \\
\hline 0 & -2.11637300 & 3.53913900 & -0.36202900 \\
\hline $\mathrm{H}$ & -3.07339400 & 3.62163500 & -0.26115900 \\
\hline
\end{tabular}

${ }^{3} \mathrm{C} 14-01$

$\begin{array}{llll}\mathrm{C} & 3.95183500 & -1.51790700 & 1.38436600 \\ \mathrm{H} & 4.15236100 & -1.34618200 & 2.44694200 \\ \mathrm{H} & 3.29793500 & -2.36044200 & 1.12965600\end{array}$


${ }^{3} \mathrm{C} 14-02$

$\mathrm{C}$
$\mathrm{H}$
$\mathrm{H}$
$\mathrm{C}$
$\mathrm{H}$
$\mathrm{H}$
$\mathrm{O}$
$\mathrm{O}$

$$
\begin{array}{r}
-3.91714900 \\
-3.60346800 \\
-3.87974900 \\
-4.36423000 \\
-0.63175600 \\
-4.38698500 \\
1.76221700
\end{array}
$$$$
-1.86464400
$$$$
-0.98274000
$$$$
0.12040100
$$$$
3.20670000
$$$$
0.08139200
$$$$
1.26827500
$$$$
2.30451000
$$

0.43022300

1.11349100

0.73498600

$-1.53014700$

$-1.56920200$

0.53149300

$-0.66691400$

1.68756000

0.92759600

$-1.12712800$

$-1.30973400$

2.94020100

2.73905900

$-1.52869700$

0.76463500

1.00072600

$-0.86522400$

2.35954200

1.86107100

$-0.84136900$

$-1.06823100$

3.11784100

3.99380600

$-1.28494100$

$-0.60864800$

$-1.72888900$

$-1.41008300$

$-2.82804100$

$-1.05194600$

$-1.25301400$

$-1.49627600$

$-1.13672400$

$-1.86512500$

$-1.87141500$

2. 30451000

0.24048600

$-0.28474500$

1.33381100

$-0.42952100$

0.21629700

$-1.52682000$

$-1.32909700$

0.02726200 
0

Si

Si

0

0

$\mathrm{H}$

0

$\mathrm{H}$

0

0

0

0

0

Si

0

$\mathrm{H}$

0

H

V

Si

C

$\mathrm{H}$

$\mathrm{H}$

C

$\mathrm{H}$

$\mathrm{H}$

Si

0

$\mathrm{H}$

${ }^{3} \mathrm{~A} 15$

V

0

0

0

Si

Si

Si

0

0

0

0

$\mathrm{H}$
1.85572200

2.27070800

2.32965000

2.77471100

3.51771900

3.43368700

3.57276800

4.45195300

$-1.30143900$

$-1.30777800$

$-0.38393000$

1.04215100

1.08435400

$-0.48857300$

$-1.23211100$

$-1.51628700$

$-1.20690700$

$-0.70549500$

$-1.89500400$

$-0.51329300$

$-3.41541800$

$-3.38952000$

$-3.32046500$

$-4.68179400$

$-4.86895600$

$-5.59616400$

1.35657300

1.86250100

2.79915400
1.27137400

$-0.30840400$

$-0.32697800$

$-0.79567000$

$-0.33235000$

$-0.95439800$

$-0.47470800$

$-0.25219300$

$-0.06721400$

$-0.05693200$

$-2.09919100$

$-1.24359300$

$-1.21009400$

$-1.45988100$

$-2.66364700$

$-2.38299000$

$-2.52184900$

$-3.32075600$

0.86847700

$-1.49418700$

2. 30880100

2.75521100

3.13184500

1.45823000

1.32378100

1.89639500

2.12360500

3.67969000

3.86565400
1.32030300

$-1.53906200$

1.49095500

$-0.03688300$

$-2.59912900$

$-3.33271200$

2. 54369100

2. 21365800

$-1.50591100$

1.50859800

0.02520000

$-2.07912800$

2.06103900

1.55956800

$-2.42216900$

$-3.30090300$

2. 58832000

2.79414400

$-0.00031200$

$-1.51034600$

$-0.01531700$

$-1.03069400$

0.71781500

0.19670900

1.27510900

$-0.24468700$

0.00939000

0.01295600

0.15525600

0.69233500

0.46749200

$-0.06415600$

$-0.56885600$

1.07972100

$-1.34938700$

$-0.43656300$

0.86153700

1.45104200

0.23093800

$-1.40913100$

$-0.18013200$

$-2.12302500$

0.56588100

$-1.32352400$

$-1.98640300$

0.35477800

1.49203100

$-1.32419600$

$-1.87637100$

$-0.12557100$

$-2.17105700$

$-1.10743100$

$-1.36274700$

$-2.79064500$

0.98561000

0.15918300

$-2.04788300$

$-1.30580500$

1.27882800

$-1.51985900$

$-3.51513800$

$-0.23963000$

$-0.66890400$

$-3.96389500$

$-0.32451100$ 


\begin{tabular}{|c|c|c|c|}
\hline 0 & -2.99341500 & 1.21184400 & -2.57003400 \\
\hline $\mathrm{H}$ & -3.88872000 & 0.85848800 & -2.64313700 \\
\hline 0 & -2.78013300 & 0.79925500 & 2.87465100 \\
\hline $\mathrm{H}$ & -2.17000500 & 1.12210100 & 3.54931300 \\
\hline C & 5.08694600 & 0.50367300 & -0.01653200 \\
\hline $\mathrm{H}$ & 5.76691700 & 0.21521900 & 0.80862300 \\
\hline $\mathrm{H}$ & 5.65005800 & 0.33085400 & -0.95433200 \\
\hline $\mathrm{H}$ & 4.94612100 & 1.59882800 & 0.06526500 \\
\hline C & 2.39545100 & 0.03661400 & 1.75327400 \\
\hline $\mathrm{H}$ & 2.41119300 & 1.09105600 & 2.07748300 \\
\hline $\mathrm{H}$ & 1.49319500 & -0.43718200 & 2.16555300 \\
\hline $\mathrm{H}$ & 3.23110500 & -0.44148400 & 2.30447300 \\
\hline C & 3.59188600 & -2.50885500 & 0.03897900 \\
\hline $\mathrm{H}$ & 4.17086200 & -2.88242200 & -0.82798200 \\
\hline $\mathrm{H}$ & 4.10693900 & -2.88474300 & 0.94410600 \\
\hline $\mathrm{H}$ & 2.60038300 & -2.99533400 & 0.00236100 \\
\hline C & 2.37712800 & 0.03309100 & -1.71644700 \\
\hline $\mathrm{H}$ & 2.65184900 & -0.92500100 & -2.19607500 \\
\hline $\mathrm{H}$ & 1.36982400 & 0.21759000 & -2.12769000 \\
\hline $\mathrm{H}$ & 3.02034900 & 0.82014600 & -2.14413900 \\
\hline $\mathrm{Al}$ & 3.36855600 & -0.52864200 & 0.03240300 \\
\hline C & 0.90280600 & 3.15073100 & 0.41844500 \\
\hline $\mathrm{H}$ & -0.16029000 & 3.26613600 & 0.19835500 \\
\hline $\mathrm{H}$ & 1.19748000 & 3.21484500 & 1.46915100 \\
\hline C & 1.79612800 & 2.92208600 & -0.55491800 \\
\hline $\mathrm{H}$ & 2.86202500 & 2.80091900 & -0.34384400 \\
\hline $\mathrm{H}$ & 1.48313200 & 2.86614000 & -1.60072800 \\
\hline \multicolumn{4}{|c|}{ A15-16TS } \\
\hline v & 0.11665000 & 0.86435900 & -0.04418600 \\
\hline 0 & 1.70465700 & 1.89307700 & -0.16874500 \\
\hline 0 & 0.44152300 & -0.38995000 & -1.40972400 \\
\hline 0 & 0.51658100 & -0.13152000 & 1.50596500 \\
\hline Si & 3.11917600 & 1.08056400 & -0.13323700 \\
\hline Si & 1.86885300 & -1.18734300 & -1.33786500 \\
\hline Si & 1.95375600 & -0.92687800 & 1.51281000 \\
\hline 0 & 3.19992600 & 0.16683100 & 1.27989200 \\
\hline 0 & 3.12230400 & -0.06727600 & -1.36353800 \\
\hline 0 & 2.01233800 & -1.92830400 & 0.16532100 \\
\hline 0 & 2.21814400 & -1.78068600 & 2.89967400 \\
\hline $\mathrm{H}$ & 1.40811200 & -1.93886400 & 3.40058600 \\
\hline 0 & 4.42422600 & 2.08244100 & -0.24880300 \\
\hline $\mathrm{H}$ & 5.27378000 & 1.63945200 & -0.13255600 \\
\hline 0 & 2.02363700 & -2.29353100 & -2.54954300 \\
\hline
\end{tabular}


$\mathrm{H}$

C

$\mathrm{H}$

$\mathrm{H}$

$\mathrm{H}$

C

$\mathrm{H}$

$\mathrm{H}$

$\mathrm{H}$

C

$\mathrm{H}$

$\mathrm{H}$

$\mathrm{H}$

C

$\mathrm{H}$

$\mathrm{H}$

$\mathrm{H}$

Al

C

$\mathrm{H}$

$\mathrm{H}$

C

$\mathrm{H}$

$\mathrm{H}$

${ }^{3} \mathrm{~A} 16$

$\mathrm{V}$

0

0

0

Si

Si

Si

0

0

0

0

$\mathrm{H}$

0

$\mathrm{H}$

0

$\mathrm{H}$

C

$\mathrm{H}$
2.75555300

$-5.37807800$

$-4.92812600$

$-5.12619500$

$-6.47606600$

$-5.10576500$

$-6.09228100$

$-5.09497300$

$-4.35433100$

$-2.62165700$

$-1.98265100$

$-2.02907100$

$-2.97450900$

$-5.67561300$

$-5.23121400$

$-5.67293200$

$-6.73986400$

$-4.82251800$

$-2.78510300$

$-3.44108600$

$-3.28929500$

$-1.47344500$

$-1.05722000$

$-1.19156500$
$-2.91120400$

$-2.17312900$

$-2.93844200$

$-2.46654200$

$-2.28941400$

0.10532600

$-0.28306600$

1.17617500

$-0.39626000$

$-0.34755700$

$-0.39942400$

$-0.19071900$

$-1.39695900$

0.98181000

0.92845600

2.04656300

0.69935600

$-0.29256900$

1.90215000

1.84746300

1.99220500

2. 38759500

2.95243700

2.77729900
$-2.43052600$

0.42742900

$-0.23466500$

1.46556700

0.33302400

$-1.96688200$

$-2.28788500$

$-2.24507900$

$-2.60631300$

0.22640600

$-0.66897500$

1.13885400

0.34461800

1. 27912100

2. 29216100

0.98143100

1.40204700

$-0.01978700$

0.02960200

$-0.83912400$

0.99046800

$-0.11633000$

0.73271200

$-1.10682200$

$-0.29291200$

$-0.81896900$

$-0.44527500$

$-1.67466700$

$-0.88897200$

$-1.76481900$

$-0.21635000$

1.06264800

$-0.08253900$

$-1.22037700$

$-1.44235300$

1.10916300

$-3.05774800$

$-0.13921400$

$-1.34750800$

$-1.64653500$

1.75424000

0.27733400

$-2.62377900$

$-0.66946000$

1.42917600

$-3.64345500$

$-0.77685900$

0.09869700

$-2.73836900$

1.47295700

$-0.97358500$

$-2.33915000$

0.97709700

1.60222100

$-3.39016400$

$-1.25224200$

2.77704600

$-2.89202500$

$-1.97454000$

3.17928600

$-4.21921900$

$-0.24626900$

$-2.52040400$

$-5.07995500$

0.08655000

$-2.23813300$

$-1.50721800$

3.37408700

0.58115000

$-2.32230000$

3.77582900

0.90595800

5.56793500

2. 26842100

0.10313300

4.92162200

2.78722100

$-0.62684800$ 


$\begin{array}{lrrr}\text { H } & 5.24126000 & 2.57891400 & 1.11186000 \\ \mathrm{H} & 6.59009600 & 2.67215000 & -0.03362800 \\ \mathrm{C} & 5.40375100 & -0.48056100 & -1.92440700 \\ \mathrm{H} & 6.40065100 & -0.55861800 & -2.40076500 \\ \mathrm{H} & 4.98624600 & -1.50256600 & -1.90039400 \\ \mathrm{H} & 4.77237200 & 0.11697000 & -2.60554000 \\ \mathrm{C} & 2.88370900 & -0.06853700 & 0.64550800 \\ \mathrm{H} & 3.32980600 & 0.35224900 & -0.28122300 \\ \mathrm{H} & 2.00760500 & 0.57812500 & 0.81787900 \\ \mathrm{H} & 3.56312900 & 0.06306100 & 1.50505300 \\ \mathrm{C} & 6.26339400 & -0.82502400 & 1.33368700 \\ \mathrm{H} & 6.12115000 & -0.38742900 & 2.33793800 \\ \mathrm{H} & 5.80955600 & -1.83181200 & 1.34245900 \\ \mathrm{H} & 7.35325400 & -0.97568000 & 1.20520000 \\ \mathrm{Al} & 5.55531700 & 0.30841400 & -0.12172800 \\ \mathrm{C} & 2.49471700 & -1.53428300 & 0.42600500 \\ \mathrm{H} & 3.42132800 & -2.13659600 & 0.32912600 \\ \mathrm{H} & 2.00158600 & -1.90275100 & 1.34515800 \\ \mathrm{C} & 1.56212000 & -1.73272800 & -0.77736200 \\ \mathrm{H} & 1.38372900 & -2.81477700 & -0.94946600 \\ \mathrm{H} & 2.03961900 & -1.34178500 & -1.70095800\end{array}$

${ }^{3} \mathrm{~A} 17$

$\begin{array}{lrrr}\text { V } & -0.81058900 & -0.02613900 & -0.36898700 \\ \text { O } & 0.44428100 & 0.60786100 & -1.69748000 \\ \text { O } & 0.06975600 & -1.66278400 & -0.04404300 \\ \text { O } & -0.16150100 & 1.04392100 & 1.04699800 \\ \text { Si } & 2.03170200 & 0.61771300 & -1.37294400 \\ \text { Si } & 1.66264500 & -1.63292800 & 0.31012200 \\ \text { Si } & 1.44791200 & 1.01621100 & 1.36718700 \\ \text { O } & 2.31246500 & 1.51875800 & 0.02603800 \\ \text { O } & 2.52118500 & -0.94129700 & -0.95611800 \\ \text { O } & 1.91365400 & -0.58031900 & 1.59977300 \\ \text { O } & 1.84599200 & 1.93886000 & 2.67909200 \\ \text { H } & 1.06277100 & 2.26627100 & 3.13909100 \\ \text { O } & 2.95024400 & 1.20377800 & -2.61559500 \\ \text { H } & 3.88226000 & 1.31388900 & -2.39074500 \\ \text { O } & 2.24272500 & -3.13771800 & 0.66478500 \\ \text { H } & 3.17963500 & -3.14979100 & 0.89577900 \\ \text { C } & -5.01855500 & -1.21407200 & -0.66148500 \\ \text { H } & -5.74457900 & -0.70427500 & -1.32351800 \\ \text { H } & -4.60612200 & -2.06558800 & -1.23588600 \\ \text { H } & -5.60611700 & -1.65314400 & 0.16763400 \\ \text { C } & -4.14018000 & 1.76334100 & 0.74262400\end{array}$




\begin{tabular}{|c|c|c|c|}
\hline $\mathrm{H}$ & -4.78430900 & 1.66258000 & 1.63736000 \\
\hline $\mathrm{H}$ & -3.26314900 & 2.36244300 & 1.05046800 \\
\hline $\mathrm{H}$ & -4.70693500 & 2.37343100 & 0.01385300 \\
\hline C & -2.47143100 & 0.53265300 & -1.76368800 \\
\hline $\mathrm{H}$ & -1.99222000 & 1.51280600 & -1.93628700 \\
\hline $\mathrm{H}$ & -2.07053500 & -0.16368800 & -2.52478000 \\
\hline $\mathrm{H}$ & -3.52714000 & 0.67765500 & -2.05642200 \\
\hline C & -2.46203200 & -1.05917000 & 1.33804600 \\
\hline $\mathrm{H}$ & -2.33415800 & -2.12389100 & 1.08634000 \\
\hline $\mathrm{H}$ & -1.55987900 & -0.72705900 & 1.87152500 \\
\hline $\mathrm{H}$ & -3.26658000 & -1.00232500 & 2.10175900 \\
\hline $\mathrm{Al}$ & -3.58217300 & -0.00438800 & 0.01298700 \\
\hline \multicolumn{4}{|c|}{ AA18 } \\
\hline V & -0.39618300 & -0.02016800 & 0.01299900 \\
\hline 0 & 0.46682100 & -0.97751900 & -1.37008000 \\
\hline 0 & 0.48582400 & -0.71911800 & 1.53257100 \\
\hline 0 & 0.45734300 & 1.66582900 & -0.13893300 \\
\hline Si & 2.09909300 & -0.94662100 & -1.35538700 \\
\hline Si & 2.11770700 & -0.69319500 & 1.49357100 \\
\hline Si & 2.09628300 & 1.64088700 & -0.14786400 \\
\hline 0 & 2.62811400 & 0.65110700 & -1.39512500 \\
\hline 0 & 2.64982200 & -1.52050800 & 0.12779400 \\
\hline 0 & 2.64633300 & 0.88589200 & 1.24727200 \\
\hline 0 & 2.76666900 & 3.14456500 & -0.28684400 \\
\hline $\mathrm{H}$ & 2.09658400 & 3.83769400 & -0.33586400 \\
\hline 0 & 2.75311700 & -1.80313500 & -2.60510300 \\
\hline $\mathrm{H}$ & 3.71095100 & -1.71435500 & -2.68275800 \\
\hline 0 & 2.78756900 & -1.31908900 & 2.86570300 \\
\hline $\mathrm{H}$ & 3.74557100 & -1.21499300 & 2.91792100 \\
\hline C & -5.28800900 & -0.85367100 & 1.75455400 \\
\hline $\mathrm{H}$ & -4.89446900 & -1.88366300 & 1.84676900 \\
\hline $\mathrm{H}$ & -4.89375400 & -0.28305100 & 2.61667300 \\
\hline $\mathrm{H}$ & -6.38351700 & -0.92475300 & 1.90269600 \\
\hline C & -5.28393900 & -1.09861300 & -1.61049400 \\
\hline $\mathrm{H}$ & -6.37932500 & -1.18613600 & -1.75091000 \\
\hline $\mathrm{H}$ & -4.88461300 & -0.66082800 & -2.54499500 \\
\hline $\mathrm{H}$ & -4.89468000 & -2.13261200 & -1.55080200 \\
\hline C & -2.54256800 & -0.02764400 & 0.00777200 \\
\hline $\mathrm{H}$ & -2.77386300 & 0.58087000 & 0.89451600 \\
\hline $\mathrm{H}$ & -2.76500400 & 0.43603700 & -0.96468700 \\
\hline $\mathrm{H}$ & -2.77240900 & -1.10032900 & 0.08911600 \\
\hline C & -5.25904300 & 1.93598300 & -0.14097500 \\
\hline $\mathrm{H}$ & -4.86096900 & 2.52399700 & 0.70768600 \\
\hline
\end{tabular}


$\mathrm{H}$

$\mathrm{H}$

Al

${ }^{3}$ B15

V

0

0

0

Si

Si

0

0

$\mathrm{H}$

0

$\mathrm{H}$

0

0

0

0

0

0

Si

0

$\mathrm{H}$

0

H

V

Si

C

$\mathrm{H}$

$\mathrm{H}$

$\mathrm{H}$

C

$\mathrm{H}$

$\mathrm{H}$

$\mathrm{H}$

C

$\mathrm{H}$

$\mathrm{H}$

$\mathrm{H}$

C

$\mathrm{H}$

$\mathrm{H}$
$-4.85592800$

$-6.35219000$

$-4.84652800$

2.39423800

2.11454700

$-0.01063800$

$-0.15716200$

0.00224300 5

i

1.29515200

0.72042000

$-0.52745800$

0.71976800

$-0.87913900$

$-1.32392900$

$-0.07069600$

1.88368300

$-1.14084000$

1.01921500

0.25722500

1.24474900

$-0.08972700$

$-2.17506400$

$-0.77432400$

0.13071600

$-0.90745700$

1.96488100

$-0.12545300$

$-2.14755900$

0.89396200

0.52506900

$-3.61695400$

$-1.29543000$

1.46825000

$-3.69678700$

$-1.09494500$

0.84505100

$-1.45829400$

3.34709600

1.80553500

$-1.52908500$

3. 27352900

$-2.37211300$

3.18627000

$-1.59803600$

$-2.49320000$

0.41213700

$-1.73100900$

$-2.22846200$

1.65273800

0.73294000

$-3.19487800$

$-0.81236400$

0.50719100

$-1.67170500$

$-2.15795800$

$-1.29843800$

$-1.34853000$

$-0.30916300$

2.42177500

$-2.63329200$

0.29624100

1.60840600

$-4.24104500$

$-1.67215200$

$-1.82344000$

$-4.09151800$

$-2.50658400$

$-2.28521600$

$-3.78278400$

0.63789400

2.73551000

$-4.57114200$

1.07262000

2.38753800

$-1.76663600$

1.86843800

$-0.98731800$

$-2.88789300$

$-1.07092200$

$-1.10172600$

4.26739300

0.63087700

1.63039200

4.54016400

1.70166200

1.59057900

3.28730400

0.55428900

2.12817800

5.01628600

0.14665900

2. 28878400

6.05063600

$-0.13675700$

$-1.07238500$

6.83077200

$-0.66495800$

$-0.48967600$

6.05866000

$-0.58517800$

$-2.08376700$

6.40086000

0.90739400

$-1.18190100$

3.14473200

0.89039100

$-1.70882900$

3.88944500

1.70254700

$-1.69548300$

3.50701900

0.09692400

$-2.38554700$

2. 30469000

1.32451600

$-2.29802300$

3.54409900

$-2.13823600$

$-0.16656900$

2.68834400

$-2.28929900$

0.51294800

3.22229200

$-2.41059000$

$-1.18780900$ 


$\begin{array}{lrrr}\text { H } & 4.32533400 & -2.86260800 & 0.13455500 \\ \text { Al } & 4.28504500 & -0.27706900 & -0.14486900 \\ \text { C } & 2.24609600 & 3.41688700 & 0.06859700 \\ \text { H } & 1.72878600 & 3.75058000 & -0.83536800 \\ \text { H } & 3.33898200 & 3.39213100 & 0.04185700 \\ \text { C } & 1.56674600 & 3.06134300 & 1.16550100 \\ \text { H } & 2.07668800 & 2.71467800 & 2.06695200 \\ \text { H } & 0.47443800 & 3.08212200 & 1.19762400\end{array}$

${ }^{3}$ B15-16TS

\begin{tabular}{|c|c|c|c|}
\hline V & 0.70170000 & 1.49423400 & -0.13983100 \\
\hline 0 & 0.31591100 & 0.56249900 & -1.68988400 \\
\hline 0 & -0.86402400 & 2.46605500 & 0.25944300 \\
\hline 0 & 0.85584100 & 0.18551100 & 1.15273000 \\
\hline Si & -0.08044000 & -1.01317800 & -1.88426600 \\
\hline Si & 0.42686200 & -1.39249200 & 1.11005500 \\
\hline 0 & 0.27436400 & -1.84189400 & -0.48699500 \\
\hline 0 & 0.67821000 & -1.71716100 & -3.16845100 \\
\hline $\mathrm{H}$ & 1.64134700 & -1.65116300 & -3.10938900 \\
\hline 0 & 1.49235100 & -2.36252800 & 1.90284500 \\
\hline $\mathrm{H}$ & 2.41717100 & -2.08725300 & 1.84103500 \\
\hline 0 & -3.43515500 & 3.09506800 & 0.72853400 \\
\hline 0 & -2.98713800 & 0.96260400 & -1.01179400 \\
\hline 0 & -2.57463500 & 0.62960900 & 1.69085100 \\
\hline 0 & -3.11799700 & -1.43363900 & 0.11310700 \\
\hline 0 & -1.70738900 & -1.15306900 & -2.18219900 \\
\hline 0 & -1.04406000 & -1.62807400 & 1.83902600 \\
\hline Si & -2.55251300 & -1.03171500 & 1.62365800 \\
\hline 0 & -4.36938800 & -1.02204800 & -2.25020000 \\
\hline $\mathrm{H}$ & -4.16906200 & -1.50568700 & -3.06126500 \\
\hline 0 & -3.49514700 & -1.69261800 & 2.79767200 \\
\hline $\mathrm{H}$ & -4.39216900 & -1.33954600 & 2.84494400 \\
\hline V & -2.46681200 & 1.89322300 & 0.42820600 \\
\hline Si & -3.04030100 & -0.65873100 & -1.35012600 \\
\hline C & 4.06756300 & 0.42971100 & 2.09853400 \\
\hline $\mathrm{H}$ & 4.41184600 & 1.45372000 & 2.34187100 \\
\hline $\mathrm{H}$ & 2.96777300 & 0.43557000 & 2.18175600 \\
\hline $\mathrm{H}$ & 4.44826700 & -0.21439700 & 2.91604200 \\
\hline C & 6.53372600 & 0.42315600 & -0.19464300 \\
\hline $\mathrm{H}$ & 7.29752000 & 0.12759500 & 0.55139400 \\
\hline $\mathrm{H}$ & 6.86913100 & 0.00202800 & -1.16226300 \\
\hline $\mathrm{H}$ & 6.60996800 & 1.52340300 & -0.28775600 \\
\hline C & 3.41975100 & 0.05827400 & -1.37818300 \\
\hline $\mathrm{H}$ & 2.82704700 & 0.66941900 & -2.06305700 \\
\hline
\end{tabular}


H

$\mathrm{H}$

C

$\mathrm{H}$

$\mathrm{H}$

$\mathrm{H}$

Al

C

$\mathrm{H}$

$\mathrm{H}$

C

$\mathrm{H}$

${ }^{3}$ B16

$\mathrm{V}$

0

0

0

Si

Si

0

0

$\mathrm{H}$

0

$\mathrm{H}$

0

0

0

0

0

0

Si

0

$\mathrm{H}$

0

$\mathrm{H}$

V

Si

C

$\mathrm{H}$

$\mathrm{H}$

$\mathrm{H}$

C

$\mathrm{H}$
4.20428000

2.73413900

4.66683300

3.65491100

5.11366200

5.24721900

4.72933600

3.60134300

4.01715800

4.33562000

2. 37445700

2. 27409800

$-0.52958600$

$-0.67151900$

1.22780100

$-0.83991700$

$-0.24173400$

$-0.44039900$

$-0.40684300$

$-1.10415800$

$-1.96324300$

$-1.50297100$

$-2.29010700$

3.85340000

3.11828400

2.91848400

2.89452500

1.34361600

1.07349400

2.61195400

3.95977700

3.58395500

3.59032200

4.52328500

2.79661700

2.81798900

$-4.32069300$

$-4.94140100$

$-3.28092100$

$-4.36678400$

$-6.82744700$

$-7.45206100$
$-0.41950900$

$-0.70964400$

$-2.28484000$

$-2.72948900$

$-2.67885700$

$-2.72968400$

$-0.27605300$

2. 31914900

2. 44301300

2.12867200

2.90545900

3.23901200

$-1.99454500$

$-0.98405000$

0.28224700

0.33655300

$-0.65120800$

1.11409600

0.34316400

$-0.63453100$

$-1.63477700$

0.14528800

$-0.28775900$

0.75711900

1.65939500

0.14072200

0.46324100

1.58512900

2.38809300

0.03722400

0.51976100

$-1.33297500$

$-1.10800000$

1.61300900

$-1.05759900$

$-1.43361200$

$-1.74654900$

0.08264900

$-2.03887600$

2.67316700

$-2.30683100$

2. 32041800

$-1.89953000$

$-2.38215300$

$-1.38390100$

$-2.59746800$

2.92574000

$-0.15450300$

0.63240900

1.28292500

0.64728500

$-1.49012000$

$-1.62080500$

$-0.10685100$

$-1.28671500$

2.08534400

$-1.28195000$

$-2.08224700$

$-0.97906800$

$-1.61410400$

$-1.69249300$

2.38346800

$-2.26359800$

3.06481800

$-1.68193600$

$-2.73890000$

$-1.45094300$

$-2.65114200$

1.75903900

$-0.08170200$

$-0.98725000$

1.42491000

$-0.08742100$

$-2.02442700$

0.72606800

$-2.43832100$

0.28194900

$-1.98304100$

$-0.91418400$

$-2.76090800$

$-0.42343800$

0.25806700

$-1.26103200$

$-0.10998400$ 


$\begin{array}{lrrr}\text { H } & -6.96810100 & -0.39027600 & 1.35344800 \\ \text { H } & -7.26073200 & 0.50072400 & -0.16409100 \\ \text { C } & -3.87335300 & 1.68430600 & 1.00653400 \\ \text { H } & -3.74330100 & 1.94332000 & 2.07045400 \\ \text { H } & -4.95059700 & 1.46952000 & 0.87202500 \\ \text { H } & -3.26695100 & 0.77168000 & 0.86307700 \\ \text { C } & -3.88913000 & -1.98514500 & 0.80865700 \\ \text { H } & -2.91663600 & -2.21869200 & 0.34680900 \\ \text { H } & -3.72122600 & -1.56216600 & 1.81605800 \\ \text { H } & -4.43514600 & -2.93594900 & 0.95067100 \\ \text { Al } & -4.94435500 & -0.71237000 & -0.26453700 \\ \text { C } & -3.40308000 & 2.80760600 & 0.07859700 \\ \text { H } & -4.07092800 & 3.68072900 & 0.22614900 \\ \text { H } & -3.55665100 & 2.49009700 & -0.96705800 \\ \text { C } & -1.93339300 & 3.18607700 & 0.28006300 \\ \text { H } & -1.62974400 & 3.95558700 & -0.45932400 \\ \text { H } & -1.77893800 & 3.63824400 & 1.28387800\end{array}$

${ }^{3}$ B17

V

0

0

0

Si

Si

0

0

$\mathrm{H}$

0

$\mathrm{H}$

0

0

0

0

0

0

Si

0

$\mathrm{H}$

0

H

V

Si

C
$-1.66040100$

$-1.03156400$

$-0.98641700$

$-0.50987100$

0.39978200

0.87734700

1.11285100

0.22432400

0.91097700

0.94005600

1.73212800

0.32379000

1.19826000

1.74980200

3.15407500

1.45449500

2.19521300

2. 81417900

3.31970900

2.97535200

4.19255300

4.57449000

0.49758500

2.26394300

$-4.58091800$
$-0.26149300$

$-1.24928500$

1.51369900

$-1.01924200$

$-1.97557000$

$-1.84381600$

$-2.44587600$

$-3.30203700$

$-3.96722200$

$-3.09214400$

$-3.07158800$

3.84191400

1.68624600

1.75062300

0.21882200

$-0.91687800$

$-0.87786400$

0.48201000

0.84486300

0.70419300

0.82609100

1.68960700

2.27779900

0.45027000

2.01807400
$-0.27937700$

1.17021100

$-0.39530200$

$-1.58924300$

1.41878400

$-1.54560800$

$-0.00702600$

2.39080100

2. 25887400

$-2.63126500$

$-3.18177400$

$-0.12633100$

1.43947000

$-1.28030400$

0.37207000

2.16406800

$-1.90421000$

$-1.23498100$

2.95019000

3.84091600

$-2.07015300$

$-1.86986700$

$-0.10302300$

1.74698000

$-1.13708400$ 


$\begin{array}{lrrr}H & -3.60759100 & 2.44100200 & -1.44448300 \\ H & -5.15153200 & 1.80380300 & -2.06032200 \\ H & -5.12570500 & 2.82404300 & -0.60921500 \\ \text { C } & -3.10850300 & 0.71930600 & 1.60447500 \\ H & -3.70551200 & 1.59442800 & 1.93731400 \\ H & -3.25006200 & -0.08131100 & 2.34769500 \\ H & -2.07076100 & 1.06744100 & 1.69506600 \\ \text { C } & -3.41689300 & -1.01933700 & -1.36466300 \\ \text { H } & -3.07617300 & -2.05327100 & -1.16328000 \\ \text { H } & -4.51062900 & -1.12206800 & -1.48712900 \\ \text { H } & -3.03574600 & -0.73243800 & -2.36011900 \\ \text { C } & -5.95666100 & -0.49750700 & 0.70921200 \\ \text { H } & -6.73954000 & -0.65021900 & -0.05777900 \\ \text { H } & -5.71995700 & -1.49269100 & 1.13104800 \\ \text { H } & -6.42692300 & 0.08364700 & 1.52539100 \\ \text { Al } & -4.33245500 & 0.41941700 & 0.01268900\end{array}$

${ }^{3}$ B18

$\begin{array}{lrrr}\text { V } & -1.29511300 & 0.14054600 & 0.02850300 \\ \text { O } & -0.65115700 & -0.86551100 & 1.46385800 \\ \text { O } & -0.47849400 & 1.84564700 & 0.05304800 \\ \text { O } & -0.67255900 & -0.80808500 & -1.45095300 \\ \text { Si } & 0.62892900 & -1.86454900 & 1.49291600 \\ \text { Si } & 0.61385800 & -1.80702000 & -1.53908000 \\ \text { O } & 0.98955100 & -2.40773300 & -0.04055500 \\ \text { O } & 0.34371700 & -3.14976300 & 2.48953400 \\ \text { H } & 1.02405500 & -3.83369500 & 2.46208400 \\ \text { O } & 0.37403600 & -3.11135700 & -2.52402200 \\ \text { H } & 0.09466800 & -2.88439000 & -3.41948500 \\ \text { O } & 1.24818100 & 3.90605400 & 0.12434600 \\ \text { O } & 2.03222400 & 1.55605400 & 1.41812400 \\ \text { O } & 2.02774100 & 1.66316800 & -1.34882400 \\ \text { O } & 3.47539100 & -0.14107200 & -0.04428200 \\ \text { O } & 1.99836800 & -1.07922500 & 2.03920200 \\ \text { O } & 1.93767200 & -0.96451500 & -2.10514600 \\ \text { Si } & 2.87833500 & 0.25121700 & -1.54573300 \\ \text { O } & 4.25007800 & 0.32522400 & 2.45438200 \\ \text { H } & 4.06650100 & 0.24273200 & 3.39843800 \\ \text { O } & 4.10134100 & 0.43969500 & -2.63160300 \\ \text { H } & 4.64728900 & 1.22289000 & -2.48944300 \\ \text { V } & 1.15166500 & 2.33664500 & 0.06602000 \\ \text { Si } & 2.92694600 & 0.15950900 & 1.48620400 \\ \text { C } & -5.20511000 & -0.54177500 & 1.72820800 \\ \text { H } & & -1.57669400 & 1.79593600\end{array}$




$\begin{array}{lrrr}\text { H } & -5.81284900 & 0.00425300 & 2.60668100 \\ \text { H } & -7.30225700 & -0.60710900 & 1.86575600 \\ \mathrm{C} & -6.16669400 & -0.69628900 & -1.64643900 \\ \mathrm{H} & -7.25856300 & -0.73007100 & -1.82990200 \\ \mathrm{H} & -5.71221300 & -0.25849400 & -2.55507500 \\ \mathrm{H} & -5.82766700 & -1.74794700 & -1.59289500 \\ \mathrm{C} & -3.40967800 & 0.24760400 & 0.03120900 \\ \mathrm{H} & -3.63441500 & 0.79619700 & 0.95862500 \\ \mathrm{H} & -3.62631000 & 0.79515300 & -0.89890100 \\ \mathrm{H} & -3.70393200 & -0.81262600 & 0.03136200 \\ \mathrm{C} & -6.07458900 & 2.30043200 & -0.09489000 \\ \mathrm{H} & -5.64502900 & 2.85237100 & 0.76218900 \\ \mathrm{H} & -5.66150800 & 2.76094700 & -1.01199400 \\ \mathrm{H} & -7.15956600 & 2.52360900 & -0.09624000 \\ \text { Al } & -5.74720900 & 0.33852400 & 0.00254900\end{array}$

${ }^{3} \mathrm{C} 15$

V

0

0

0

Si

Si

0

0

$\mathrm{H}$

0

$\mathrm{H}$

0

0

0

0

0

Si

0

$\mathrm{H}$

0

$\mathrm{H}$

Si

C

$\mathrm{H}$

$\mathrm{H}$

$\mathrm{H}$

C
1.23422200

0.65769000

$-0.09015700$

0.94569000

$-0.17508300$

0.00264700

$-0.24285300$

0.42373000

1. 36207800

0.64292200

1.60597900

$-2.36442000$

$-2.19206600$

$-3.29911000$

$-1.74841500$

$-1.48601800$

$-2.70626700$

$-4.21942200$

$-4.13256800$

$-3.86472900$

$-4.62591200$

$-2.89606000$

4.25207200

4.56753900

3.27679000

4.98855200

5.96404300
0.71133600

$-0.94855500$

1.85876900

0.36139100

$-2.19154000$

$-0.72643600$

$-2.04650200$

$-3.67886700$

$-3.75295300$

$-1.18197300$

$-1.25152800$

0.40177300

1.74991300

$-0.65421800$

$-2.18774400$

$-0.07156300$

0.52012000

$-1.55970200$

$-2.47458600$

1.04023200

1.45873600

$-1.01237800$

0.71945000

1.77333200

0.71347900

0.24654400

$-0.30274300$
$-0.54110800$

$-1.22731600$

$-1.22499100$

1.26408500

$-0.60601100$

2.03177100

1.05614800

$-1.00715400$

$-0.78242300$

3.48487400

3.46253400

$-1.79045300$

0.47904200

0.44685900

$-1.16564800$

2. 37734500

1.46168600

$-1.93734100$

$-2.23272000$

2.50863600

2.08807100

$-1.12351800$

1.54487800

1.43437600

2.05876000

2. 22536900

$-1.12567200$ 


$\begin{array}{lrrr}\text { H } & 6.73601100 & -0.81332600 & -0.51684500 \\ \text { H } & 5.93318200 & -0.83102400 & -2.09754500 \\ \text { H } & 6.35198400 & 0.71422600 & -1.32752600 \\ \mathrm{C} & 3.08291500 & 0.75244100 & -1.77965500 \\ \mathrm{H} & 3.85583500 & 1.53542800 & -1.84878400 \\ \mathrm{H} & 3.38223800 & -0.08695400 & -2.43134400 \\ \mathrm{H} & 2.23138100 & 1.19053400 & -2.34642300 \\ \mathrm{C} & 3.44344600 & -2.15545200 & 0.00173200 \\ \mathrm{H} & 2.58654600 & -2.21583500 & 0.69355500 \\ \mathrm{H} & 3.11531100 & -2.52889400 & -0.98484800 \\ \mathrm{H} & 4.21393500 & -2.85537000 & 0.37914500 \\ \mathrm{Al} & 4.20539600 & -0.30886200 & -0.16640700 \\ \mathrm{C} & 2.30899600 & 3.40722400 & -0.12280400 \\ \mathrm{H} & 1.79812400 & 3.65555200 & -1.05706100 \\ \mathrm{H} & 3.40137600 & 3.36552400 & -0.14340400 \\ \mathrm{C} & 1.61972200 & 3.16249100 & 0.99732600 \\ \mathrm{H} & 2.12134700 & 2.89578600 & 1.93042600 \\ \mathrm{H} & 0.52778200 & 3.18948300 & 1.01609200 \\ \mathrm{Si} & -1.70755000 & 1.77872900 & -1.12156100 \\ \mathrm{O} & -2.39877800 & 3.08745900 & -1.86276700 \\ \mathrm{H} & -1.74205200 & 3.70361000 & -2.20914400\end{array}$

\section{${ }^{3} \mathrm{C} 15-16 \mathrm{TS}$}

V

0

0

0

Si

Si

0

0

$\mathrm{H}$

0

$\mathrm{H}$

0

0

0

0

0

Si

0

$\mathrm{H}$

0

$\mathrm{H}$
$-0.58515000$

$-0.24501600$

0.97812300

$-0.82574100$

0.19650100

$-0.37506000$

$-0.18230000$

$-0.48949100$

$-1.45555600$

$-1.44607200$

$-2.35391600$

3.01284100

2. 54272500

3. 21766000

1.83882900

1.09112600

2. 58171200

4.48962600

4.32970600

3.50348100

4.38845900
1.54010600

0.57273800

2.47805400

0.22550900

$-0.99146200$

$-1.34252700$

$-1.81755000$

$-1.73857700$

$-1.70686700$

$-2.32291700$

$-1.99039300$

1.05726800

0.75077100

$-1.34014800$

$-1.09384300$

$-1.55231200$

$-0.90500100$

$-0.83218100$

$-1.37060300$

$-1.47906200$

$-1.09785800$
0.17840800

1.72922600

$-0.23350300$

$-1.11427000$

1.88812700

$-1.09145400$

0.49501300

3.19065800

3.16801200

$-1.86921600$

$-1.87312500$

0.91617600

$-1.66425700$

$-0.18661400$

2.12855500

$-1.84630900$

$-1.66060100$

2.15881500

2.94387000

$-2.89585800$

$-2.94914300$ 


\begin{tabular}{|c|c|c|c|}
\hline Si & 3.13359400 & -0.55023700 & 1.26951300 \\
\hline Si & 2.47669900 & 1.87845200 & -0.43552400 \\
\hline 0 & 3.54707900 & 3.09230900 & -0.77449800 \\
\hline $\mathrm{H}$ & 3.12421500 & 3.95857800 & -0.81679100 \\
\hline C & -4.63775800 & -2.29433800 & -0.14717100 \\
\hline $\mathrm{H}$ & -5.00931300 & -2.64358300 & 0.83577900 \\
\hline $\mathrm{H}$ & -5.27863300 & -2.77754200 & -0.91067400 \\
\hline $\mathrm{H}$ & -3.62980300 & -2.73579600 & -0.26525600 \\
\hline C & -3.35544200 & 0.12958900 & 1.35744200 \\
\hline $\mathrm{H}$ & -4.12516500 & -0.34032700 & 1.99910500 \\
\hline $\mathrm{H}$ & -2.65860800 & -0.63836100 & 0.98417700 \\
\hline $\mathrm{H}$ & -2.76756800 & 0.76815900 & 2.02114000 \\
\hline C & -6.47630500 & 0.46933100 & 0.19525000 \\
\hline $\mathrm{H}$ & -6.52696400 & 1.57495300 & 0.20890900 \\
\hline $\mathrm{H}$ & -7.25679600 & 0.13824600 & -0.51803900 \\
\hline $\mathrm{H}$ & -6.80620100 & 0.12620000 & 1.19498000 \\
\hline C & -4.04064700 & 0.29317100 & -2.12521100 \\
\hline $\mathrm{H}$ & -4.31438300 & -0.46517500 & -2.88548400 \\
\hline $\mathrm{H}$ & -4.48992100 & 1.24140400 & -2.47998000 \\
\hline $\mathrm{H}$ & -2.94573100 & 0.42102200 & -2.17473700 \\
\hline Al & -4.69018600 & -0.29269100 & -0.31717400 \\
\hline C & -2.28986900 & 2.94055500 & 0.26831900 \\
\hline $\mathrm{H}$ & -1.90106400 & 3.61104300 & 1.04974400 \\
\hline $\mathrm{H}$ & -2.11061800 & 3.27174900 & -0.76670000 \\
\hline C & -3.53352000 & 2.33059200 & 0.50332900 \\
\hline $\mathrm{H}$ & -4.16376700 & 2.09262600 & -0.35161900 \\
\hline $\mathrm{H}$ & -4.06749500 & 2.48205500 & 1.44178800 \\
\hline \multicolumn{4}{|c|}{${ }^{3} \mathrm{C} 16$} \\
\hline v & -0.42996000 & 1.69647200 & 0.15140900 \\
\hline 0 & -0.59319000 & 0.47730200 & 1.59603500 \\
\hline 0 & 1.30360400 & 2.44571200 & 0.04424300 \\
\hline 0 & -0.78230100 & 0.53973600 & -1.32005000 \\
\hline Si & -0.14512900 & -1.08419100 & 1.61448900 \\
\hline Si & -0.36567400 & -1.02857100 & -1.42561800 \\
\hline 0 & -0.31366700 & -1.72967100 & 0.08584800 \\
\hline 0 & -0.97854600 & -2.03838900 & 2.68034500 \\
\hline $\mathrm{H}$ & -1.83002800 & -2.32756000 & 2.32624700 \\
\hline 0 & -1.41500900 & -1.88813500 & -2.37613000 \\
\hline $\mathrm{H}$ & -2.20922200 & -1.38158700 & -2.58681800 \\
\hline 0 & 3.09983400 & 0.75454500 & 1.20488200 \\
\hline 0 & 2.88566400 & 0.76841000 & -1.42849100 \\
\hline 0 & 3.00870700 & -1.53347800 & -0.12603500 \\
\hline 0 & 1.45222900 & -1.24492000 & 2.06953500 \\
\hline
\end{tabular}


1.15318300

2.67442500

4.08802700

3.74950400

3.68325000

4.60432300

2.90013600

2. 76098100

3.95694600

3.60587400

$-3.77688700$

$-3.59642000$

$-4.32765600$

$-2.80921900$

$-3.77091500$

$-4.84446100$

$-3.14189700$

$-3.65276300$

$-6.72451000$

$-7.17438600$

$-7.33474800$

$-6.86533300$

$-4.23776000$

$-4.28381100$

$-4.87056700$

$-3.20135700$

$-4.83580300$

$-1.86626200$

$-1.74708800$

$-1.58333100$

$-3.32272100$

$-3.45257300$

$-4.02218800$

$-1.55897500$

0.07624400

$-0.44483000$

$-0.71133200$

$-1.13755400$

0.81808100

0.17630100

0.90846300

0.76139800

1.36772000
$-1.23545600$

$-0.86178400$

$-1.46828600$

$-2.04091000$

$-1.45655800$

$-1.18823500$

$-0.87063700$

1.76165100

2.90304600

3.79859700

$-2.00241600$

$-1.58357200$

$-2.95026100$

$-2.24007300$

1.63724800

1.40563500

0.74355300

1.87292700

$-0.46293100$

0.45531900

$-1.30992400$

$-0.43642400$

$-0.09381800$

$-0.92019300$

0.71423500

0.28604000

$-0.72044400$

3.20978100

3.65166300

4.00428400

2.79273300

2.48804700

3.64037000
$-0.96982100$

1.73325900

$-0.83345600$

$-1.93573500$

$-1.69149800$

$-2.39833000$

$-3.29522800$

$-3.98939400$
$-2.07942600$

$-1.61026500$

2. 36648800

3.06583700

$-2.76950300$

$-2.66463100$

1.39181600

$-0.08781500$

$-0.18936400$

$-0.11180900$

0.80136000

1.80830600

0.94548800

0.33158800

0.98229800

0.83855000

0.82061000

2.05294800

0.26821700

$-0.14969500$

$-0.10272500$

1.36386100

$-2.02665200$

$-2.76353900$

$-2.43318900$

$-1.99460800$

$-0.25797100$

0.31186000

1.32508700

$-0.40942300$

0.08271200

$-0.97007200$

0.23691200

0.47135800

1.61490800

0.66780700

$-1.11472200$

1.36112300

$-1.55176200$

$-0.24068800$

2. 30607100

2.02007700 
0

$\mathrm{H}$

0

0

0

0

0

Si

0

$\mathrm{H}$

0

$\mathrm{H}$

Si

Si

0

$\mathrm{H}$

C

$\mathrm{H}$

$\mathrm{H}$

$\mathrm{H}$

C

$\mathrm{H}$

$\mathrm{H}$

$\mathrm{H}$

C

$\mathrm{H}$

$\mathrm{H}$

$\mathrm{H}$

C

$\mathrm{H}$

$\mathrm{H}$

$\mathrm{H}$

Al

${ }^{3} \mathrm{C} 18$

V

0

0

0

Si

Si

0

0

$\mathrm{H}$
$-0.20989300$

$-1.10532400$

1.94498700

1.27024800

3. 20081900

2. 25921100

1. 31182400

2. 20955500

4.37280300

4.52129800

3.09152800

3.57658500

2.93547800

0.79805600

0.91762200

0.05129900

$-5.65365700$

$-5.19587100$

$-6.14487000$

$-6.46411400$

$-3.32002800$

$-4.40247500$

$-2.99534000$

$-2.88075300$

$-3.03599900$

$-2.10280400$

$-3.76631600$

$-2.89031000$

$-4.97474600$

$-5.58094500$

$-4.13922700$

$-5.61202000$

$-4.29849900$

\subsection{0}

0.56648700

0.39250800

0.68592800

$-0.72282200$

$-0.61674900$

$-1.04197100$

$-0.49933800$

$-1.15937100$
$-2.87332400$

$-2.76583500$

1.53967700

1.81148300

0.13127600

$-1.14943700$

$-0.69311900$

0.57983700

0.46101200

$-0.16989600$

1.06780100

1.89116300

0.23787400

2. 23479100

3.87579400

4.29674700

1.63126800

2. 31573300

2. 27395500

1.09005400

$-0.51478700$

$-0.54475300$

$-1.56938700$

$-0.01284900$

1.59678600

1.31310100

1.71700000

2.59051900

$-1.13818300$

$-0.82996300$

$-1.74736600$

$-1.81321800$

0.42583400

$-2.64370000$

$-2.98842900$

1. 36225200

$-1.17837300$

$-0.49808000$

1.70192500

$-2.25452000$

$-1.75674500$

1.91128300

2.62618500

$-3.05918300$

$-2.92430100$

1.13573400

0.37097100

0.54670600

0.61043500

0.81161500

1.55138000

0.05585900

1.33636100

1.68347900

1.90451000

1.70977100

2.56701100

$-1.08935400$

$-1.59615500$

$-1.91766600$

$-0.63774900$

$-1.05065000$

$-1.92415400$

$-1.44187900$

$-0.44851800$

$-0.02142300$

0.22421000

$-0.12018500$

$-0.87617300$

$-1.49463300$

1.90204400

$-0.17494800$

$-0.71940800$

1.41474900

$-1.85777000$

$-1.43536400$

$-1.67745400$

1.59566200

$-2.34019200$

0.12681100

$-3.19073700$

$-2.38834100$

$-3.88217400$

$-2.25565300$ 


$$
\begin{array}{r}
-0.35597400 \\
0.57896700 \\
-2.02807800 \\
-1.93463000 \\
-3.52342300 \\
-2.10081100
\end{array}
$$$$
-1.94410100
$$$$
-2.84512900
$$$$
-4.24097900
$$$$
-4.20066900
$$$$
-4.00305000
$$$$
-4.50939900
$$$$
-2.96361100
$$$$
-1.17871000
$$$$
-1.37966200
$$$$
-0.54281500
$$$$
6.07280300
$$

5.62299000

7.16461100

5.72631400

3.35318800

3.55026000

3.61135800

3.59676400

5.99554100

5.60906200

7.07958100

5.53935700

6.17930900

7.28038700

5.79304000

5.82086400

5.65920300
$-2.87974700$

$-2.97624700$

1.58558800

1.75227400

$-0.02484600$

$-1.07097400$

$-0.82970500$

0.40392500

0.43919400

$-0.11621000$

0.71831400

1.52376600

0.22672300

2. 30057900

3.93930400

4.40891300

2.29966600

2.85980600

2.47809500

2.78705100

0.33255300

0.91429300

$-0.73540500$

0.83478900

$-0.55187200$

$-1.58833500$

$-0.61855600$

$-0.01690200$

$-0.68742800$

$-0.76635800$

$-1.72413300$

$-0.22424600$

0.34953600

1.57189300

1.50187900

$-0.19491100$

1.00700700

0.15667100

$-1.50528700$

$-0.33754500$

$-0.16104900$

$-1.14972400$
2.70139300

2.92077900

$-1.38362000$

1.25000800

0.09421900

$-1.97064100$

2.13476400

1.55113500

$-2.46406000$

$-3.25215300$

2. 67718400

2. 51490100

$-1.44541700$

$-0.13532100$

$-0.22078600$

$-0.32385000$

$-0.06955200$

0.77202000

$-0.01288400$

$-1.00052000$

$-0.13405900$

0.77875200

$-0.08349500$

$-1.08153900$

1.73195700

1.76123700

1.94972900

2.58639800

$-1.63502300$

$-1.72721000$

$-1.62329600$

$-2.57369800$

$-0.01474700$

0.01484500

$-1.17340600$

$-0.46868300$

1.63311000

$-1.16268100$

$-0.50801200$

1.57621200

0.39966500

$-1.53316200$ 


$\begin{array}{lrrr}\text { O } & 0.65706500 & -1.61721100 & 1.01957600 \\ \text { O } & 2.30919600 & -0.71540800 & 3.01552400 \\ H & 1.89439100 & -0.26940400 & 3.76461000 \\ \text { O } & 3.52332000 & 0.24390200 & -2.20389800 \\ H & 4.14847600 & -0.48785100 & -2.12965000 \\ \text { O } & -0.72557200 & -2.89237300 & -0.99437400 \\ H & -1.66339000 & -2.95846200 & -0.76179700 \\ \text { C } & -3.77201900 & 0.91455800 & -1.42008200 \\ H & -3.35614700 & 1.93883200 & -1.44910700 \\ H & -3.63771100 & 0.48874800 & -2.43312000 \\ H & -4.86329400 & 1.02346800 & -1.26593500 \\ C & -2.91416500 & 0.53718900 & 1.84121500 \\ H & -3.93721500 & 0.55748600 & 2.26550800 \\ H & -2.29627700 & -0.07986500 & 2.52005200 \\ H & -2.52841100 & 1.57006100 & 1.92790600 \\ \text { C } & -1.09502500 & 3.40346700 & -0.08712000 \\ H & -2.00689100 & 3.48717700 & 0.53115500 \\ H & -0.38312800 & 4.19188900 & 0.23227800 \\ H & -1.37070100 & 3.60568800 & -1.14113200 \\ \text { C } & -3.53383100 & -2.12057700 & -0.04845300 \\ H & -3.52963700 & -2.59757800 & -1.04923500 \\ H & -3.02126500 & -2.79964400 & 0.66113200 \\ H & -4.59654300 & -2.13312300 & 0.26347400 \\ \text { Al } & -2.90529000 & -0.20534100 & -0.01715500\end{array}$

$\begin{array}{lrrr}{ }^{3} \mathrm{~A} 20 & & & \\ \mathrm{~V} & 0.15387700 & 1.37345000 & -0.34258100 \\ 0 & 1.56708600 & 0.71510300 & -1.39296500 \\ 0 & -1.15157600 & -0.24958700 & -0.57634900 \\ \mathrm{O} & 0.65439500 & 0.80813900 & 1.43581400 \\ \mathrm{Si} & 2.12774900 & -0.78998000 & -1.10986900 \\ \mathrm{Si} & -0.46746600 & -1.76435800 & -0.40071300 \\ \mathrm{Si} & 1.24684500 & -0.69808700 & 1.61976600 \\ \mathrm{O} & 2.49560900 & -0.94795300 & 0.51983600 \\ \mathrm{O} & 0.87346000 & -1.87470800 & -1.37583200 \\ \mathrm{O} & 0.08905700 & -1.83231700 & 1.17319700 \\ \mathrm{O} & 1.80688700 & -1.00817600 & 3.14298300 \\ \mathrm{H} & 1.45471100 & -0.38920500 & 3.79470000 \\ 0 & 3.43526600 & -1.15030500 & -2.04604200 \\ \mathrm{H} & 3.81037600 & -2.02512700 & -1.88555300 \\ \mathrm{O} & -1.47864400 & -2.99778400 & -0.78205200 \\ \mathrm{H} & -2.36024400 & -2.90076200 & -0.39343500 \\ \mathrm{C} & -3.98136300 & 0.76860900 & -1.49329100 \\ \mathrm{H} & -3.78238900 & 1.81878000 & -1.77235000\end{array}$




$\begin{array}{lrrr}\text { H } & -3.78988800 & 0.15738200 & -2.39639900 \\ \text { H } & -5.06822800 & 0.69323900 & -1.29132800 \\ \mathrm{C} & -2.62948500 & 1.40445800 & 1.58154200 \\ \mathrm{H} & -3.60620300 & 1.67884400 & 2.02785200 \\ \mathrm{H} & -2.02820400 & 0.93004700 & 2.37748200 \\ \mathrm{H} & -2.12857400 & 2.36048200 & 1.34247400 \\ \mathrm{C} & -0.95538300 & 2.83864600 & -1.36722600 \\ \mathrm{H} & -1.43502000 & 2.30410900 & -2.20979600 \\ \mathrm{H} & -1.75552100 & 3.34187800 & -0.79632700 \\ \mathrm{H} & -0.29380400 & 3.61749600 & -1.79179600 \\ \mathrm{C} & -3.67984100 & -1.58345000 & 0.80868300 \\ \mathrm{H} & -4.05887600 & -2.32058200 & 0.07014700 \\ \mathrm{H} & -3.04033400 & -2.12208000 & 1.53353300 \\ \mathrm{H} & -4.58075400 & -1.28455400 & 1.38081800 \\ \mathrm{Al} & -2.90507700 & 0.12926400 & 0.06019600 \\ \mathrm{C} & 1.18790100 & 3.66460300 & 0.79214400 \\ \mathrm{H} & 0.87281500 & 3.30786200 & 1.77640700 \\ \mathrm{H} & 0.62613400 & 4.49453600 & 0.35569700 \\ \mathrm{C} & 2.22858700 & 3.10456900 & 0.15873300 \\ \mathrm{H} & 2.55182900 & 3.44133200 & -0.83017000 \\ \mathrm{H} & 2.78441100 & 2.27659100 & 0.60405000\end{array}$

${ }^{3}$ A20-21TS

$\begin{array}{lrrr}\text { V } & -0.33134100 & 1.37623300 & 0.15688600 \\ \text { O } & 1.22724800 & 1.43103300 & -0.96870200 \\ \text { O } & -0.96596800 & -0.53483900 & -0.63511100 \\ \text { O } & 0.41557000 & 0.43478800 & 1.69246100 \\ \text { Si } & 2.24297900 & 0.17277000 & -1.07140600 \\ \text { Si } & 0.15801900 & -1.74821500 & -0.78578800 \\ \text { Si } & 1.49334900 & -0.76003500 & 1.52082900 \\ \text { O } & 2.71996200 & -0.31348500 & 0.46282200 \\ \text { O } & 1.41564200 & -1.16539700 & -1.69443200 \\ \text { O } & 0.75780100 & -2.06137100 & 0.73742100 \\ \text { O } & 2.16098400 & -1.27528200 & 2.94579200 \\ \text { H } & 1.73197200 & -0.86242300 & 3.70552900 \\ \text { O } & 3.56156200 & 0.49466800 & -2.01437300 \\ \text { H } & 4.17394000 & -0.24757700 & -2.08996600 \\ \text { O } & -0.46543700 & -3.10418700 & -1.48588000 \\ \text { H } & -1.21765500 & -3.46871000 & -1.00023700 \\ \text { C } & -3.98337300 & -0.36499400 & -1.41982500 \\ \text { H } & -3.82295600 & 0.58811900 & -1.95716700 \\ \text { H } & -3.91697000 & -1.16846800 & -2.17902000 \\ \text { H } & -5.03338800 & -0.35700200 & -1.06636600 \\ \text { C } & -2.67053800 & 0.84607400 & 1.44033800\end{array}$




$\begin{array}{lrrr}H & -3.72904400 & 1.16209400 & 1.52253500 \\ H & -2.36591400 & 0.43196200 & 2.41641500 \\ H & -2.14582200 & 1.82186300 & 1.37267000 \\ \text { C } & -1.57290700 & 2.59888300 & -1.25441300 \\ H & -1.43057900 & 3.39541800 & -1.99345100 \\ H & -1.35005700 & 1.65477900 & -1.79276600 \\ H & -2.61925200 & 2.59171400 & -0.91705400 \\ \text { C } & -2.87050700 & -2.43389100 & 0.95410600 \\ H & -3.06448800 & -3.27153200 & 0.25344400 \\ H & -1.98216600 & -2.70419900 & 1.55426100 \\ H & -3.73110400 & -2.43621600 & 1.65168900 \\ \text { Al } & -2.67743700 & -0.65051900 & 0.06061200 \\ \text { C } & 0.18376900 & 3.23747000 & 1.18098500 \\ H & 1.26925700 & 3.11778500 & 1.20590600 \\ H & -0.32819300 & 3.25059900 & 2.14872700 \\ \text { C } & -0.42548100 & 3.81624200 & 0.05487300 \\ H & -1.33985300 & 4.40378500 & 0.17798000 \\ H & 0.22425500 & 4.15197300 & -0.75674800\end{array}$

${ }^{3} \mathrm{~A} 21$

$\begin{array}{lrrr}\text { V } & -0.28319100 & 1.18009400 & 0.62720300 \\ \text { O } & 1.00240600 & 1.88410000 & -0.57095900 \\ \text { O } & -0.85059500 & -0.49910800 & -0.42062200 \\ \text { Si } & 0.80619000 & 0.30925500 & 1.91544800 \\ \text { Si } & 2.18485400 & 0.88789700 & -1.07336200 \\ \text { Si } & 0.45602400 & -1.40191400 & -0.97347500 \\ \text { O } & 1.99723300 & -0.65117200 & 1.33599900 \\ \text { O } & 2.95366900 & 0.20301600 & 0.25451000 \\ \text { O } & 1.45989900 & -0.42099000 & -1.85454900 \\ \text { O } & 1.30618700 & -1.85837100 & 0.38775000 \\ \text { H } & 2.94883400 & -1.30849300 & 2.51230000 \\ \text { O } & 2.56256200 & -1.22350500 & 3.39291700 \\ \text { H } & 3.27260400 & 1.62798300 & -2.06448900 \\ \text { O } & 4.01534200 & 1.07093200 & -2.32857500 \\ \text { H } & -0.00530100 & -2.67232400 & -1.90278800 \\ \text { C } & -0.69108600 & -3.21947500 & -1.49232700 \\ H & -3.73018600 & -1.08143900 & -1.49186400 \\ H & -4.02628900 & -0.02879400 & -1.65175200 \\ \text { H } & -3.26673000 & -1.42788100 & -2.43575000 \\ \text { C } & -4.66749100 & -1.66008400 & -1.37413600 \\ \text { H } & -2.99364600 & -0.45758900 & 1.77810300 \\ \text { H } & -3.69306100 & -1.11280100 & 2.33370100 \\ \text { H } & -2.12994000 & -0.30143800 & 2.45395400 \\ & -3.50001000 & 0.51682300 & 1.66832700\end{array}$


$-2.41559300$

1.86083000

$-1.29748500$

$-3.78503400$

2.29775900

$-0.26110400$

$-2.07757100$

$-3.29204800$

0.35990500

$-2.10360900$

$-3.95686900$

$-0.52910600$

$-1.12616200$

$-3.48453500$

0.89012900

$-2.87369700$

$-3.69144200$

1.01891700

$-2.49435300$

$-1.33824200$

0.05187800

$-1.45678900$

2.77274400

1.23907700

$-0.74227200$

3.39795600

1.81618900

$-2.25905100$

2.46546600

1.93689200

$-2.01718500$

3.55256800

0.04365100

$-2.56634000$

4.45607400

0.38049000

$-1.17677000$

3.92404000

$-0.57025300$

${ }^{3}$ B19$$
\text { V }
$$$$
0
$$

0

0

Si

Si

0

0

$\mathrm{H}$

0

$\mathrm{H}$

0

0

0

0

0

0

Si

0

$\mathrm{H}$

0

H

V

Si

C

$\mathrm{H}$

$\mathrm{H}$
$-1.07752000$

0.10694300

$-0.09375700$

$-1.77773300$

0.92224800

$-0.98928200$

0.07983900

1.13935500

1.67380600

$-2.03471400$

$-2.69897300$

1. 58105200

2.58373200

0.78849200

2.42234300

2.42186900

$-0.17044600$

1.08076300

4.66056900

5.10993500

1.32143800

1.83364100

1.18920900

3.02496700

$-4.86143000$

$-4.85642900$

$-4.84108100$
0.81458200

2.20380100

$-0.66003200$

0.36645900

2. 53121500

0.68938300

1.91712300

4.15532100

4.40786200

1.12757700

0.45305800

$-2.73053200$

$-0.50375900$

$-2.18864900$

$-0.60694900$

1.80553900

$-0.67868800$

$-1.57963600$

0.32713700

0.97233500

$-2.78240400$

$-3.53104000$

$-1.57472700$

0.27353000

0.50027000

1. 32614300

0.96843100
$-1.58934600$

$-1.21162700$

$-2.23301700$

0.22846200

0.14838600

1.66545300

1.45131200

0.33319800

1.09603000

2.85323000

3.05454800

$-2.58809300$

$-1.23064900$

0.04551500

1.42821600

0.17712600

2.11244900

1.55848300

0.35128700

$-0.20856400$

2.65301900

2. 32297200

$-1.59684200$

0.16555700

$-0.11815500$

0.61953500

$-1.11845300$ 
$\mathrm{H}$

C

$\mathrm{H}$

$\mathrm{H}$

$\mathrm{H}$

C

$\mathrm{H}$

$\mathrm{H}$

$\mathrm{H}$

C

$\mathrm{H}$

$\mathrm{H}$

$\mathrm{H}$

Al

${ }^{3}$ B20

$\mathrm{V}$

0

0

0

Si

Si

0

0

$\mathrm{H}$

0

$\mathrm{H}$

0

0

0

0

0

0

Si

0

$\mathrm{H}$

0

H

V

Si

C

$\mathrm{H}$

$\mathrm{H}$

$\mathrm{H}$
$-5.84625800$

$-3.48350600$

$-4.13011800$

$-2.53221200$

$-3.98571800$

$-2.51877100$

$-2.02480400$

$-3.25799000$

$-3.04809500$

$-2.91261900$

$-2.67969800$

$-2.03826300$

$-3.75862800$

$-3.35412600$

0.00254500

$-1.60283100$

$-2.49621800$

$-1.97135100$

$-0.99080000$

1.47801100

1.79099200

0.69062700

2. 35104100

$-2.08827200$

$-1.67112100$

$-2.69881000$

$-2.78693000$

$-0.75961000$

$-1.07705000$

$-1.67549700$

0.19353300

0.16377400

$-2.12042800$

$-1.11923400$

$-0.37730900$

$-0.96930000$

1.82485400

$-1.66674600$

0.15961100

1.02981200

$-1.22015200$

$-0.90570400$

1.07073600

0.18169500

0.16030200

1.35166700

$-2.06613500$

1.82531800

$-1.99730100$

$-2.91803800$

1. 22210700

2.35794900

0.67304700

2.51084600

2.47860600

$-0.05017500$

1.12468300

4.64859700

5.05598900

1.46657700

0.68654200

0.93441500

3.00219200

$-1.56873300$

1.71562100

1.64718500

0.00790400

$-0.46857200$

1.70689300

1.70841600

$-0.75728600$

2. 29303300

2.45301700

0.14068100

$-0.71828900$

4.04547200

4.59766800

0.03994800

0.13725100

0.96264500

1.85161800

0.06741500

0.95488200

.02194900
2.03686200

1.92880700

2.46037200

2.81447000

$-2.89237000$

$-3.83366700$

$-3.12498400$

$-2.46668400$

$-1.20402400$

$-2.20040400$

$-0.91212400$

$-1.35470200$

0.21534700

$-0.50720700$

$-2.15206400$

$-1.67452100$

$-2.51571800$

$-3.53159300$

$-4.20960100$

$-2.72027300$

$-2.42710700$

3.73873100

1. 20997500

1.58691700

$-0.33989900$

$-1.47434400$

$-0.95940900$

0.17830400

$-0.17269800$

$-0.33868200$

0.40068900

0.54283700

2.19169300

$-0.19365600$

$-1.01005200$

$-4.63813700$

$-1.67429600$

$-4.58913300$

$-5.60510800$

$-1.66048000$

$-0.52140100$ 


$\begin{array}{lrrr}\text { C } & -2.60781900 & 2.78830300 & 0.95586100 \\ \text { H } & -3.43437600 & 3.29526700 & 1.49182100 \\ \text { H } & -1.74862500 & 2.75154500 & 1.64970900 \\ H & -2.32412800 & 3.44628100 & 0.11457600 \\ \mathrm{C} & -2.83583400 & -2.77429900 & -0.02179900 \\ \mathrm{H} & -3.18065600 & -3.17224700 & 0.94976100 \\ \mathrm{H} & -3.64051900 & -2.15054800 & -0.44930600 \\ \mathrm{H} & -2.65841400 & -3.62725900 & -0.70293300 \\ \mathrm{C} & -3.63447600 & -0.20470800 & 1.97160600 \\ \mathrm{H} & -2.76356300 & -0.64746400 & 2.48581100 \\ \mathrm{H} & -4.13601400 & 0.43645300 & 2.72351600 \\ \mathrm{H} & -4.33534000 & -1.02422900 & 1.73660000 \\ \mathrm{Al} & -3.17704600 & 0.95193900 & 0.41128500 \\ \mathrm{C} & 0.54149700 & -3.63073200 & 1.54918800 \\ \mathrm{H} & 0.41165900 & -3.46808400 & 2.62247700 \\ \mathrm{H} & 1.38395900 & -3.11940200 & 1.07532400 \\ \mathrm{C} & -0.30339200 & -4.39161400 & 0.84270000 \\ \mathrm{H} & -0.16945100 & -4.53338400 & -0.23251300 \\ \mathrm{H} & -1.15287600 & -4.89543700 & 1.31274900\end{array}$

${ }^{3}$ B20-21TS

$\begin{array}{lrrr}\text { V } & 1.71775400 & -1.03101500 & -0.58489000 \\ \text { O } & 0.66840100 & -2.16731100 & 0.51718300 \\ \text { O } & 0.42239500 & -0.27795000 & -1.81760300 \\ \text { O } & 1.44361400 & 0.64342400 & 0.69584600 \\ \text { Si } & -0.48472200 & -1.95541500 & 1.62118300 \\ \text { Si } & 0.37499900 & 0.94347700 & 1.91740900 \\ \text { O } & -0.30134900 & -0.48317600 & 2.38786300 \\ \text { O } & -0.42646600 & -3.16214200 & 2.75484900 \\ \text { H } & -1.16081500 & -3.14341200 & 3.38064700 \\ \text { O } & 1.14837900 & 1.69144900 & 3.17080400 \\ \text { H } & 2.11040800 & 1.61868600 & 3.12485200 \\ \text { O } & -1.30339000 & 0.47183600 & -3.71963300 \\ \text { O } & -2.33230700 & -0.90485700 & -1.49450400 \\ \text { O } & -1.50736100 & 1.75241400 & -1.22419000 \\ \text { O } & -2.97481300 & 0.55855000 & 0.65419400 \\ \text { O } & -2.02462600 & -1.96230000 & 0.98057800 \\ \text { O } & -0.82781600 & 1.96207800 & 1.42607600 \\ \text { Si } & -2.04890600 & 1.90168000 & 0.32927600 \\ \text { O } & -4.50616600 & -1.52078600 & 0.04666200 \\ \text { H } & -4.59949600 & -2.45515400 & -0.17618000 \\ \text { O } & -2.93228800 & 3.28102300 & 0.50579600 \\ \text { H } & -3.30974300 & 3.60336800 & -0.32203600 \\ \text { V } & -1.12391300 & 0.25385500 & -2.16719900\end{array}$


Si

C

$\mathrm{H}$

$\mathrm{H}$

$\mathrm{H}$

C

$\mathrm{H}$

$\mathrm{H}$

$\mathrm{H}$

C

$\mathrm{H}$

$\mathrm{H}$

$\mathrm{H}$

C

$\mathrm{H}$

$\mathrm{H}$

$\mathrm{H}$

Al

C

$\mathrm{H}$

$\mathrm{H}$

C

$\mathrm{H}$

$\mathrm{H}$

${ }^{3}$ B21

$\mathrm{V}$

0

0

0

Si

Si

0

0

$\mathrm{H}$

0

$\mathrm{H}$

0

0

0

0

0

0

Si
$-2.94782400$

4.09364100

3.82117400

4.41707500

4.99720900

1.60248100

2.20085900

0.71464900

1.23425900

3.63214700

4.45235800

3.07794700

4.04213700

3.22236300

3.23968600

2.71301200

4.29569600

2.60719400

1.98168400

2. 23363600

1.00568000

3.01003400

2. 78283500

4.04611100

$-1.23773500$

$-0.29816800$

$-0.07470300$

$-1.52640700$

0.52997600

$-0.70174500$

$-0.06239500$

0.38615600

0.97597100

$-1.71443600$

$-2.54425000$

2.03511000

2. 58010400

1.42345500

2.96570300

2.15260600

0.54044300

1.85524500
$-0.97592800$

2.10951900

2.80579400

1.16120000

2.54376300

3.44370400

4.13929100

3.15719700

4.02541300

$-1.73796600$

$-1.10053700$

$-1.20897600$

$-2.63281700$

0.76057900

$-0.31894600$

1.21904800

1.02649800

1.83094000

$-2.60171900$

$-2.15343900$

$-3.08953300$

$-3.00346100$

$-3.81298200$

$-2.98736100$

$-0.81483900$

$-2.25699900$

1.17215200

0.13060200

0.44295200

0.37473400

2.33458700

$-2.39747700$

$-0.42284900$

0.22940600

$-0.93941500$

$-1.27665200$

$-1.87274300$

$-3.91491300$

$-2.02396400$

$-4.10023100$

$-1.57043700$

0.52477400

$-2.31144700$

0.96116800

1.46678900

$-3.13075400$

$-2.88841500$

$-0.49965700$

3.31920600

1.88048600

1.40414000

0.48839400

0.61671300

$-2.07938300$

$-1.05333900$

1. 31654600

$-0.74484700$

1.71266500

$-1.88190200$

$-0.97456100$ 
${ }^{3} \mathrm{C} 19$

V

0

0

0

Si

Si

0

0

$\mathrm{H}$

0

$\mathrm{H}$

0

0
4.67362300

4.86440000

2.55381200

1.94807900

1.47188600

3.09232900

$-4.10884400$

$-3.90712300$

$-4.50365400$

$-4.95843400$

$-1.40131800$

$-1.93973600$

$-0.51304600$

$-1.04317800$

$-4.23697300$

$-4.94334900$

$-3.47941000$

$-4.78830500$

$-3.11795300$

$-2.32782700$

$-3.35798300$

$-4.01918800$

$-2.58348400$

$-2.86663700$

$-3.55318300$

$-2.40243800$

$-3.59767400$

$-2.88511400$

$-4.38649700$
$-1.33191100$

$-2.16823800$

3.07297400

3.82213100

0.77239700

$-0.87590900$

1.81088500

2. 21069700

0.78482000

2.41837400

3.52377700

4.47275100

3.51754200

3.56631400

$-1.95083700$

$-1.17009300$

$-1.45288100$

$-2.65563400$

1.83282900

1.48761500

2.86372900

1.22601900

1.96090700

$-1.71186300$

$-0.96791400$

$-2.27493400$

$-2.66155500$

$-3.42021300$

$-3.22392400$
$-0.16214100$

0.28031300

$-1.57542800$

$-1.65317700$

2.02556000

$-0.12584100$

$-1.39781200$

$-2.41325300$

$-1.51539600$

$-1.02883200$

$-0.47210400$

$-0.28141700$

0.18416000

$-1.51695400$

$-0.10275400$

0.22606000

$-0.72984400$

$-0.74780100$

1.82503400

2.51762400

2.15299800

2.01858200

$-0.09338900$

2.05070000

2.49492300

2.88842400

1.09517600

0.72578600

1.63526300

$-0.98320200$

0.89189100

$-1.55834000$

0.18745700

2.27634100

$-1.06236400$

$-0.01240400$

$-0.52562400$

$-2.28880700$

$-1.74009300$

0.36006100

0.23604500

1.02107400

2.44702800

0.30969400

$-0.92997500$

0.53793800

1.68357700

0.17833000

1.74551200

1.56925200

1.30216300

4.03566300

0.65997300

1.69186300

4.19098100

1.52900100

$-1.94554200$

0.90510600

2.92130400

$-2.64866700$

0.25217700

3.04885400

2.54128600

$-0.55261300$

$-1.33019100$

0.74411700

$-2.15384300$

$-0.24153100$ 
0

0

0

Si

0

$\mathrm{H}$

0

$\mathrm{H}$

Si

Si

0

$\mathrm{H}$

C

$\mathrm{H}$

$\mathrm{H}$

$\mathrm{H}$

C

$\mathrm{H}$

$\mathrm{H}$

$\mathrm{H}$

C

$\mathrm{H}$

$\mathrm{H}$

$\mathrm{H}$

C

$\mathrm{H}$

$\mathrm{H}$

$\mathrm{H}$

Al

${ }^{3} \mathrm{C} 20$

$\mathrm{V}$

0

0

0

Si

Si

0

0

$\mathrm{H}$

0

$\mathrm{H}$

0

0
2.45506200

2.49611800

$-0.14180400$

1.07008300

4.69202700

5.06923700

1.25629900

1.79269000

3.04815800

1.17934300

1.62225700

0.97602300

$-3.54509900$

$-4.02937500$

$-4.23312400$

$-2.62009000$

$-2.41738500$

$-3.16409300$

$-2.94818700$

$-1.91634700$

$-4.81740200$

$-4.78086300$

$-5.81597100$

$-4.79250100$

$-2.94682700$

$-3.81841800$

$-2.67420700$

$-2.10360300$

$-3.34878300$
$-0.83783900$

1.66500200

$-0.88628300$

$-1.75862200$

0.15247000

1.03743100

$-3.09991000$

$-3.79641900$

0.12308600

$-1.44680600$

$-2.63017300$

$-2.75351900$

$-1.63071200$

$-1.04004400$

$-2.48358200$

$-2.07161000$

1.63656800

0.87764300

2.48463900

2.01202700

0.61435900

1.10619300

0.14160100

1.42142800

$-1.99242200$

$-2.64692700$

$-1.53456800$

$-2.64950000$

$-0.69968400$
1.30045800

0.24850200

2.00174800

1.32752200

0.17315900

0.09757000

2.25943300

1.86107800

0.08749900

$-1.69657200$

$-2.76047900$

$-3.46708100$

1.91435300

2.71964500

1.74871100

2. 32820700

$-2.84429200$

$-3.14094900$

$-2.37117400$

$-3.75915400$

$-0.16046400$

$-1.14871500$

$-0.07911000$

0.59732900

$-1.32745900$

$-1.52451200$

$-2.29528000$

$-1.04532500$

0.13798200

$$
0.88785200
$$

$-1.73029100$

$-0.05607400$

$-0.36324800$

$-1.96017100$

1.31941000

0.18964800

$-1.22363000$

$-1.74029400$

1.64047300

0.09278200

0.50682100

$-1.21009900$

$-0.88899300$

2.19725500

0.93641700

1.15900500

1.57593100

$-0.22511300$

0.42190300

2.48345300

$-1.77988400$

$-1.48428100$

3.62908600

$-1.09574400$

$-1.86678500$

4.19221100

2.06386800

1.81461400

2.57743300

2.97954700

1.65711700

2. 30371600

$-2.34766300$

$-0.36374300$

$-1.31942700$

$-0.45582100$

1.43188500

$-1.73484200$ 


\begin{tabular}{|c|c|c|c|}
\hline 0 & -2.38824700 & 1.93731200 & 0.00711300 \\
\hline 0 & -2.56698700 & -0.37126700 & 1.39553900 \\
\hline 0 & 0.18386500 & 2.36390900 & 0.72454200 \\
\hline Si & -0.89868400 & 2.44670500 & -0.51102500 \\
\hline 0 & -4.63048100 & 0.39910500 & -0.10519100 \\
\hline $\mathrm{H}$ & -5.07360600 & 0.18183400 & 0.72429600 \\
\hline 0 & -1.01937700 & 4.00725100 & -1.01198300 \\
\hline $\mathrm{H}$ & -0.17462600 & 4.47632700 & -1.02080700 \\
\hline Si & -2.98899300 & 0.39018600 & -0.00134200 \\
\hline Si & -0.91431000 & -0.11717500 & -2.15119400 \\
\hline 0 & -1.25383600 & -0.17344500 & -3.76820100 \\
\hline $\mathrm{H}$ & -0.58390700 & -0.65378600 & -4.27024100 \\
\hline C & 2.85061500 & 2.53451800 & -1.08676300 \\
\hline $\mathrm{H}$ & 2.58974400 & 3.25784500 & -0.29329400 \\
\hline $\mathrm{H}$ & 3.74776500 & 2.93635900 & -1.59838500 \\
\hline $\mathrm{H}$ & 2.02984800 & 2.54334400 & -1.82669300 \\
\hline C & 2.51297500 & -3.02306100 & 0.18788100 \\
\hline $\mathrm{H}$ & 3.34406500 & -2.52481000 & 0.72137200 \\
\hline $\mathrm{H}$ & 2.20267300 & -3.90704900 & 0.77707500 \\
\hline $\mathrm{H}$ & 2.89809300 & -3.37818700 & -0.78508800 \\
\hline C & 4.64303200 & 0.65379600 & 1.00691000 \\
\hline $\mathrm{H}$ & 4.53122000 & -0.19049400 & 1.71373900 \\
\hline $\mathrm{H}$ & 5.63541600 & 0.52157600 & 0.53298000 \\
\hline $\mathrm{H}$ & 4.74873900 & 1.57708800 & 1.61433400 \\
\hline C & 3.57213600 & -0.59387000 & -1.92315500 \\
\hline $\mathrm{H}$ & 4.17713900 & -1.47184700 & -1.63858600 \\
\hline $\mathrm{H}$ & 2.65003300 & -0.96506600 & -2.40401000 \\
\hline $\mathrm{H}$ & 4.13857400 & -0.05576800 & -2.70901500 \\
\hline Al & 3.22415200 & 0.68204200 & -0.42998100 \\
\hline C & 0.04493800 & -4.27481900 & -1.45387200 \\
\hline $\mathrm{H}$ & 0.68459600 & -5.11448700 & -1.16579700 \\
\hline $\mathrm{H}$ & 0.33323500 & -3.70964400 & -2.34396300 \\
\hline C & -1.03132700 & -3.93360100 & -0.73821600 \\
\hline $\mathrm{H}$ & -1.65897300 & -3.08264700 & -1.01490700 \\
\hline $\mathrm{H}$ & -1.31084000 & -4.47253700 & 0.17137400 \\
\hline \multicolumn{4}{|c|}{${ }^{3} \mathrm{C} 20-21 \mathrm{TS}$} \\
\hline V & -1.58824900 & -1.11735700 & 0.60133800 \\
\hline 0 & -0.46225700 & -2.13570100 & -0.55263900 \\
\hline 0 & -0.42442400 & -0.33168200 & 1.89537200 \\
\hline 0 & -1.43925700 & 0.63055400 & -0.63389600 \\
\hline $\mathrm{Si}$ & 0.65656500 & -1.81989200 & -1.67396000 \\
\hline Si & -0.35771100 & 1.03779900 & -1.80871200 \\
\hline 0 & 0.35195000 & -0.33609100 & -2.37765600 \\
\hline
\end{tabular}




\begin{tabular}{|c|c|c|c|}
\hline 0 & 0.71069000 & -2.98007300 & -2.85827900 \\
\hline $\mathrm{H}$ & -0.13765100 & -3.42602400 & -2.96876400 \\
\hline 0 & -1.11519600 & 1.87150900 & -3.01877900 \\
\hline $\mathrm{H}$ & -2.07780200 & 1.79725300 & -2.98738800 \\
\hline 0 & 2.22810400 & -0.86720100 & 1.50009000 \\
\hline 0 & 1.39166000 & 1.63110100 & 1.39665300 \\
\hline 0 & 3.06842300 & 0.72625300 & -0.45060200 \\
\hline 0 & 2.20419200 & -1.76018600 & -1.07012200 \\
\hline 0 & 0.82460400 & 2.03395500 & -1.22215100 \\
\hline Si & 2.02042000 & 1.95677500 & -0.08981100 \\
\hline 0 & 4.52316100 & -1.47219800 & 0.20171600 \\
\hline $\mathrm{H}$ & 4.72293600 & -2.15532100 & -0.45005400 \\
\hline 0 & 2.84695500 & 3.37962500 & -0.11360600 \\
\hline $\mathrm{H}$ & 2.30931200 & 4.13695200 & -0.37718200 \\
\hline Si & 3.00259200 & -0.85676100 & 0.04968900 \\
\hline Si & 1.07838800 & 0.16672100 & 2.14768000 \\
\hline 0 & 1.40659900 & 0.34249300 & 3.76299200 \\
\hline $\mathrm{H}$ & 0.63123800 & 0.14428800 & 4.30220700 \\
\hline C & -1.72802400 & 3.38103900 & 0.82144200 \\
\hline $\mathrm{H}$ & -1.34036400 & 3.96955200 & -0.03164700 \\
\hline $\mathrm{H}$ & -2.37244600 & 4.06386500 & 1.40843300 \\
\hline $\mathrm{H}$ & -0.85980500 & 3.12156900 & 1.45297800 \\
\hline C & -3.47062300 & -1.84195600 & -0.44846300 \\
\hline $\mathrm{H}$ & -2.93131800 & -1.23704700 & -1.20180000 \\
\hline $\mathrm{H}$ & -3.83429100 & -2.72559600 & -0.98520700 \\
\hline $\mathrm{H}$ & -4.32799700 & -1.26738500 & -0.07083100 \\
\hline C & -4.12270600 & 2.03697100 & -1.13207500 \\
\hline $\mathrm{H}$ & -4.41622900 & 1.09942800 & -1.64328200 \\
\hline $\mathrm{H}$ & -5.04608200 & 2.43664000 & -0.66900000 \\
\hline $\mathrm{H}$ & -3.84939500 & 2.76806100 & -1.91922200 \\
\hline C & -3.31216200 & 0.60386900 & 1.77951900 \\
\hline $\mathrm{H}$ & -4.41291000 & 0.72154500 & 1.79119100 \\
\hline $\mathrm{H}$ & -3.18167900 & -0.47684500 & 1.98537100 \\
\hline $\mathrm{H}$ & -2.91008200 & 1.08934600 & 2.68574000 \\
\hline$A l$ & -2.66701900 & 1.73853500 & 0.21583500 \\
\hline C & -1.84805400 & -2.79741500 & 1.96796900 \\
\hline $\mathrm{H}$ & -2.15132800 & -2.41580300 & 2.94822000 \\
\hline $\mathrm{H}$ & -0.84851100 & -3.23527000 & 1.90697100 \\
\hline C & -2.82918200 & -3.18123600 & 1.03281000 \\
\hline $\mathrm{H}$ & -2.54861200 & -3.93426500 & 0.29265500 \\
\hline $\mathrm{H}$ & -3.87670000 & -3.22816100 & 1.34561000 \\
\hline \multicolumn{4}{|c|}{${ }^{3} \mathrm{C} 21$} \\
\hline V & -1.12898900 & -0.87158000 & 1.17432100 \\
\hline
\end{tabular}


0

0

0

Si

Si

0

0

$\mathrm{H}$

0

H

0

0

0

0

0

Si

0

H

0

H

Si

Si

0

$\mathrm{H}$

C

$\mathrm{H}$

$\mathrm{H}$

$\mathrm{H}$

C

$\mathrm{H}$

$\mathrm{H}$

$\mathrm{H}$

C

$\mathrm{H}$

$\mathrm{H}$

$\mathrm{H}$

C

$\mathrm{H}$

$\mathrm{H}$

$\mathrm{H}$

Al

C

$\mathrm{H}$

$\mathrm{H}$

\begin{tabular}{rrr}
-0.20395000 & -2.28378600 & 0.34004400 \\
0.01335300 & 0.01757900 & 2.36910600 \\
-1.49759600 & 0.35459500 & -0.39608400 \\
0.67316400 & -2.31396600 & -1.02206700 \\
-0.65086200 & 0.29106000 & -1.83843200 \\
0.00633500 & -1.19731700 & -2.07021000 \\
0.73788300 & -3.79264200 & -1.75598900 \\
-0.11894100 & -4.23359600 & -1.80983400 \\
-1.64677700 & 0.64557800 & -3.09611300 \\
-2.48419200 & 1.06015000 & -2.84191100 \\
2.57151300 & -0.41064200 & 1.48839900 \\
1.31916000 & 1.80568100 & 0.80268900 \\
3.03420900 & 0.68369000 & -0.88511700 \\
2.26060300 & -1.90190800 & -0.76929700 \\
0.58348300 & 1.38671700 & -1.76495300 \\
1.82474000 & 1.80754200 & -0.76912600 \\
4.70894900 & -1.23707000 & 0.04960300 \\
4.87332500 & -2.05521000 & -0.43544800 \\
2.41260000 & 3.27152100 & -1.22723500 \\
1.72438500 & 3.93713000 & -1.36124100 \\
3.14577200 & -0.73209700 & -0.02255600 \\
1.45021500 & 0.69669900 & 2.04573400 \\
2.07783700 & 1.43176600 & 3.38540600 \\
1.44146100 & 1.48727000 & 4.10897900 \\
-1.46377800 & 3.50817100 & -0.39170400 \\
-1.08431200 & 3.56052100 & -1.42860300 \\
-2.04450800 & 4.43559300 & -0.21943200 \\
-0.59291800 & 3.54051200 & 0.28694200 \\
-4.13573500 & -2.02073800 & -0.08352000 \\
-3.38724100 & -1.50198300 & -0.70444900 \\
-4.69378200 & -2.71026800 & -0.74002800 \\
-4.84068200 & -1.25080600 & 0.27254000 \\
-4.11706900 & 1.74143200 & -1.34548300 \\
-4.46624000 & 0.70548900 & -1.51113300 \\
-4.99148700 & 2.29374900 & -0.94871600 \\
-3.93645100 & 2.19507500 & -2.34229500 \\
-3.13395600 & 1.73982800 & 1.88084400 \\
-4.00401400 & 1.08544800 & 2.06293500 \\
-2.32463800 & 1.41188600 & 2.55935900 \\
-3.42218000 & 2.75171000 & 2.22839800 \\
-2.59580300 & 1.89935100 & -0.03351700 \\
\hline & -1.82565000 & 2.05876400 \\
\hline & -1.10425000 & 2.52921700 \\
\hline
\end{tabular}


C

$\mathrm{H}$

H
$-3.47991100$

$-2.76699100$

$-4.26089900$
$-2.75653200$

$-3.50197300$

$-3.33882600$
1.09030500

0.69361100

1.62168100 